\title{
STOCHASTIC CALCULUS OVER SYMMETRIC MARKOV PROCESSES WITH TIME REVERSAL
}

\author{
K. KUWAE
}

\begin{abstract}
We develop stochastic calculus for symmetric Markov processes in terms of time reversal operators. For this, we introduce the notion of the progressively additive functional in the strong sense with time-reversible defining sets. Most additive functionals can be regarded as such functionals. We obtain a refined formula between stochastic integrals by martingale additive functionals and those by Nakao's divergence-like continuous additive functionals of zero energy. As an application, we give a stochastic characterization of harmonic functions on a domain with respect to the infinitesimal generator of semigroup on $L^{2}$-space obtained by lower-order perturbations.
\end{abstract}

\section{Contents}

1. Introduction . . . . . . . . . . . . . . . . . . . 91

2. Preliminary facts . . . . . . . . . . . . . . . . . . . 93

3. Nakao integrals . . . . . . . . . . . . . . . . . . . . . . . . 103

4. Progressively additive functionals in the strong sense . . . . . . . . . . . . . . 105

5. Main theorems . . . . . . . . . . . . . . . . . . . . . . 114

6. Proofs of Theorems 5.1 and 5.2 . . . . . . . . . . . . . . . . . . 122

7. Application: Boundary value problem for infinitesimal generator of perturbed

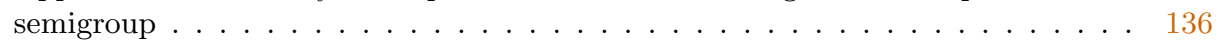

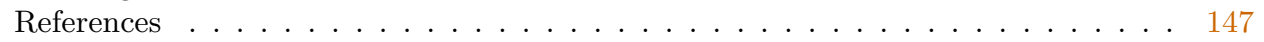

\section{$\S 1$. Introduction}

The author's previous article [13] refined stochastic calculus over symmetric Markov processes and investigated stochastic integrals by extending Nakao's divergence-like continuous additive functional (CAF) of zero energy in the framework of general symmetric Markov processes without using time reversal operators. In the present article we develop the results in [13] in terms of time reversal operators. For this we introduce a refined notion

\footnotetext{
Received November 15, 2012. Revised November 9, 2013. Accepted February 18, 2014. 2010 Mathematics Subject Classification. Primary 31C25; Secondary 60J25, 60J45,
} $60 \mathrm{~J} 75$. 
of additive functionals, so-called progressively additive functionals in the strong sense (PrAFSs) with the notion of time-reversible defining sets. The notion of PrAFSs on $\llbracket 0, \zeta \llbracket$ with time-reversible defining sets is also introduced. Hereafter, we call PrAFSs (on $\llbracket 0, \zeta \llbracket$ ) with time-reversible defining sets reversible PrAFSs (on $\llbracket 0, \zeta \llbracket$ ) (see Definitions 4.6 and 4.8). We show that most additive functionals that appeared in the textbook by Fukushima, Ōshima, and Takeda [8] can be regarded as reversible PrAFSs (see Lemmas 6.1 and 6.2, Corollaries 6.1 and 6.2). The weaker notion, progressively additive functionals (PrAFs), was formulated in [4] as a natural extension of the positive progressively additive functionals admitting no exceptional set that were introduced by Walsh in [22]. In [22], Walsh considered the positive PrAF for the study of dual additive functionals admitting no exceptional set in the framework of a pair of Markov processes in duality. One of our aims is to establish the dual PrAF admitting exceptional sets in the framework of symmetric Markov processes associated with regular Dirichlet forms. However, the definition of the time reversal operator treated in [22] is slightly different from ours, and his PrAFs require nonnegativity and strict measurability, which are very restrictive for our purposes. The dual PrAF of any PrAF obtained in [4, Theorem 2.18] allows only $\mathfrak{m}$-negligible sets because of the lack of time-reversible defining sets, which is also restrictive for obtaining refined formulas. For this reason we introduce the reversible PrAFS (on $\llbracket 0, \zeta \llbracket$ ) (see Lemma 4.3).

Our first result is that any locally square integrable martingale additive functionals (MAFs) on $\llbracket 0, \zeta \llbracket$ introduced in [4] can be regarded as reversible PrAFSs on $\llbracket 0, \zeta \llbracket$ (Theorem 5.1). Second, we prove a formula between locally square integrable MAFs and its extension of Nakao's divergence-like CAF in terms of PrAFSs on $\llbracket 0, \zeta \llbracket$ with time reversal operators (Theorem 5.2). As corollaries, we obtain quasieverywhere (q.e.) refinements of Lyons-Zheng decompositions for Fisk-Stratonovich-type integrals (Theorem 5.3 and Corollaries 5.1-5.5). Our Lyons-Zheng decompositions for Fisk-Stratonovichtype integrals by purely discontinuous Dirichlet processes are new even under the law $\mathbf{P}_{\mathfrak{m}}$. Our Theorem 5.2 also connects two kinds of extensions of Nakao's divergence-like CAF of zero energy and its stochastic integrals developed, respectively, in [4] and [13] (Theorem 5.4).

The time reversibility of the defining sets of PrAFS plays a crucial role in our Theorems 5.1 and 5.2 instead of the $\mathbf{P}_{\mathfrak{m}}$-invariance of the time reversal operator $r_{t}$ on $\{t<\zeta\}$ (see (2.3)). In particular, the dual additive functional of the given reversible PrAFS defined by the time reversal operator can be 
regarded as a relaxed $\operatorname{Pr} A F\left(\operatorname{Pr} A F^{*}\right)$ in Lemma 4.3 (see Definitions 4.1 and 4.4 for the definition of $\left.\operatorname{PrAF}^{*}\right)$. From this we obtain that the dual multiplicative functional on $\llbracket 0, \zeta \llbracket$ of any Doléans-Dade stochastic exponential of locally square integrable MAFs on $\llbracket 0, \zeta \llbracket$ can be regarded as a relaxed progressively multiplicative functional (PrMF) on $\llbracket 0, \zeta \llbracket$ (Lemma 7.1), which together with Theorem 5.1 yields the $\mathcal{E}$-quasicontinuity of strongly continuous semigroups on $L^{2}$-space associated with a lower bounded quadratic form obtained from the combinations of the Feynman-Kac transform and Girsanov transform developed in [3] (Theorem 7.3). This $\mathcal{E}$-quasicontinuity enables us to obtain a stochastic characterization of the harmonic function on a domain (Theorem 7.4 and Corollary 7.3).

Let us briefly outline the organization of this paper. In Sections 2 and 3 we give some results in [13] with several corrections as errata. In Section 4 we introduce the notion of PrAFSs and reversible defining sets. In Section 5 we prove Theorems 5.1 and 5.2 as our main theorems and prove Theorems 5.3 and 5.4. We show some examples. In Section 6 we present Theorems 5.1 and 5.2. In Section 7 we prove Theorems 7.3 and 7.4 and give an example.

\section{§2. Preliminary facts}

Let $\mathbf{X}=\left\{\Omega, \mathscr{F}_{\infty}, \mathscr{F}_{t}, X_{t}, \theta_{t}, \mathbf{P}_{x}, x \in E\right\}$ be an $\mathfrak{m}$-symmetric right Markov process on a Lusin space $E$, where $\mathfrak{m}$ is a $\sigma$-finite measure with full support on $E$. Its associated Dirichlet space $(\mathcal{E}, \mathcal{F})$ on $L^{2}(E ; \mathfrak{m})$ is known to be quasiregular (see [17]). By [6], $(\mathcal{E}, \mathcal{F})$ is quasihomeomorphic to a regular Dirichlet space on a locally compact separable metric space. Thus, using this quasihomeomorphism, without loss of generality, we may and do assume that $\mathbf{X}$ is an $\mathfrak{m}$-symmetric Hunt process on a locally compact metric space $E$ such that its associated Dirichlet space $(\mathcal{E}, \mathcal{F})$ is regular on $L^{2}(E ; \mathfrak{m})$ and that $\mathfrak{m}$ is a positive Radon measure with full topological support on $E$. Let $E_{\partial}:=E \cup\{\partial\}$ be the 1-point compactification of $E$, and let $\zeta:=\inf \{t \geq 0 \mid$ $\left.X_{t}=\partial\right\}$ be the lifetime of $\mathbf{X}$. We implicitly use the quasileft continuity up to $\infty$, which is not the usual property of right Markov processes. So the strict quasiregularity of $(\mathcal{E}, \mathcal{F})$ is essentially assumed (see [6]). However, if we restrict ourselves to stating the result that it holds up to the lifetime with probability 1 for a q.e. starting point, then the framework of quasiregular Dirichlet forms is enough.

Without loss of generality, we may assume that $\Omega$ is the canonical path space $D\left(\left[0, \infty\left[\rightarrow E_{\partial}\right)\right.\right.$ of right-continuous, left-limited $($ rcll $)$ functions from 
$\left[0, \infty\left[\right.\right.$ to $E_{\partial}$. For any $\omega \in \Omega$, we set $X_{t}(\omega):=\omega(t)$. As usual, $\mathscr{F}_{\infty}$ and $\mathscr{F}_{t}$ are the minimal completed $\sigma$-algebras obtained from $\mathscr{F}_{\infty}^{0}:=\sigma\left\{X_{s} \mid 0 \leq s<\infty\right\}$ and $\mathscr{F}_{t}^{0}:=\sigma\left\{X_{s} \mid 0 \leq s \leq t\right\}$, respectively, under $\mathbf{P}_{x}$. We set $X_{t}(\omega):=\partial$ for $t \geq \zeta(\omega)$, and we use $\theta_{t}$ to denote the shift operator defined by $\theta_{t}(\omega)(s):=$ $\omega(t+s), t, s \geq 0$. Let $\omega_{\partial}$ be the path starting from $\partial$. Then $\omega_{\partial}(s) \equiv \partial$ for all $s \in\left[0, \infty\left[\right.\right.$. Note that $\theta_{\zeta(\omega)}(\omega)=\omega_{\partial}$ for all $\omega \in \Omega$, that $\left\{\omega_{\partial}\right\} \in \mathscr{F}_{0}^{0} \subset \mathscr{F}_{t}^{0}$ for all $t>0$, and that $\mathbf{P}_{x}\left(\left\{\omega_{\partial}\right\}\right) \leq \mathbf{P}_{x}\left(X_{0}=\partial\right)=0$ for $x \in E$. Given a path $\omega \in\{t<\zeta\}$, the operator $r_{t}$ is defined by

$$
r_{t}(\omega)(s):= \begin{cases}\omega(t-s)_{-}, & \text {if } 0 \leq s \leq t, \\ \omega(0), & \text { if } s \geq t\end{cases}
$$

For a path $\omega \in\{t \geq \zeta\}$, we set $r_{t}(\omega):=\omega_{\partial}$. Here for $r>0, \omega(r)_{-}:=\lim _{s \uparrow r} \omega(s)$, and we use the convention that $\omega(0)_{-}:=\omega(0)$. We note that

$$
\begin{aligned}
& \lim _{s \downarrow 0} r_{t}(\omega)(s)=\omega(t)_{-}=r_{t}(\omega)(0) \quad \text { and } \\
& \lim _{s \uparrow t} r_{t}(\omega)(s)=\omega(0)=r_{t}(\omega)(t) .
\end{aligned}
$$

Note that $\zeta\left(r_{t} \omega\right)=\infty$ under $t<\zeta(\omega)$. It is well known that for $A \in \mathscr{F}_{t}^{0}$

$$
\mathbf{P}_{\mathfrak{m}}\left(r_{t}^{-1}(A) \cap\{t<\zeta\}\right)=\mathbf{P}_{\mathfrak{m}}(A \cap\{t<\zeta\}) .
$$

For a (nearly) Borel subset $B$ of $E, \sigma_{B}:=\inf \left\{t>0 \mid X_{t} \in B\right\}$ (the first hitting time to $B$ of $\left.X_{t}\right), \widehat{\sigma}_{B}:=\inf \left\{t>0 \mid X_{t-} \in B\right\}$ (the first hitting time to $B$ of $X_{t-}$ ), and $\tau_{B}:=\inf \left\{t>0 \mid X_{t} \notin B\right\}$ (the first exit time of $B$ ) are $\left(\mathscr{F}_{t}\right)$-stopping times. In [8, Theorem A.2.3] we see that $\mathbf{P}_{x}\left(\sigma_{B} \leq \widehat{\sigma}_{B}\right)=1$ for all $x \in E$. If $B$ is closed, then $\tau_{B}$ is an $\left(\mathscr{F}_{t+}^{0}\right)$-stopping time. Also, $\zeta$ is an $\left(\mathscr{F}_{t}^{0}\right)$-stopping time because $\{\zeta \leq t\}=\left\{X_{t}=\partial\right\} \in \mathscr{F}_{t}^{0}, t \geq 0$. The transition semigroup of $\mathbf{X},\left\{P_{t}, t \geq 0\right\}$, is defined by

$$
P_{t} f(x):=\mathbf{E}_{x}\left[f\left(X_{t}\right)\right]=\mathbf{E}_{x}\left[f\left(X_{t}\right): t<\zeta\right], \quad t \geq 0
$$

where the function $f$ is regarded to be defined on $E_{\partial}$ with $f(\partial)=0$. Each $P_{t}$ may be viewed as an operator on $L^{2}(E ; \mathfrak{m})$; collectively, these operators form a strongly continuous semigroup of self-adjoint contractions. The Dirichlet form associated with $\mathbf{X}$ is the bilinear form

$$
\mathcal{E}(u, v):=\lim _{t \downarrow 0} \frac{1}{t}\left(u-P_{t} u, v\right)_{\mathfrak{m}}
$$


defined on the space

$$
\mathcal{F}:=\left\{u \in L^{2}(E ; \mathfrak{m}) \mid \sup _{t>0} t^{-1}\left(u-P_{t} u, u\right)_{\mathfrak{m}}<\infty\right\}
$$

Here we use the notation $(f, g)_{\mathfrak{m}}:=\int_{E} f(x) g(x) \mathfrak{m}(\mathrm{d} x)$ for $f, g \in L^{2}(E ; \mathfrak{m})$.

An increasing sequence $\left\{F_{n}\right\}$ of closed sets is called an $\mathcal{E}$-nest if $\bigcup_{n=1}^{\infty} \mathcal{F}_{F_{n}}$ is $\mathcal{E}_{1}^{1 / 2}$-dense in $\mathcal{F}$, where $\mathcal{F}_{F_{n}}:=\{u \in \mathcal{F} \mid u=0 \mathfrak{m}$-almost everywhere (m-a.e.) on $\left.E \backslash F_{n}\right\}$, and a family $\left\{F_{n}\right\}$ of closed sets is an $\mathcal{E}$-nest if and only if it is a (generalized) nest, that is, if $\mathbf{P}_{x}\left(\lim _{n \rightarrow \infty} \tau_{F_{n}}=\zeta\right)=1$ q.e. $x \in E$. A function $u$ on $E$ is said to be $\mathcal{E}$-quasicontinuous if there exists an $\mathcal{E}$-nest $\left\{F_{n}\right\}$ of closed sets such that $u$ is continuous on each $F_{n}$. A subset $N$ of $E$ is called $\mathcal{E}$-polar or $\left(\mathcal{E}\right.$-)exceptional if there exists an $\mathcal{E}$-nest $\left\{F_{n}\right\}$ such that $N \subset \bigcap_{n=1}^{\infty}\left(E \backslash F_{n}\right)$; equivalently, there is a Borel set $\tilde{N}$ containing $N$ such that $\mathbf{P}_{\mathfrak{m}}\left(\sigma_{\tilde{N}}<\infty\right)=0$. A subset $G$ of $E$ is called $\mathcal{E}$-quasiopen if there exists an $\mathcal{E}$-nest $\left\{F_{n}\right\}$ of closed sets such that, for each $n \in \mathbb{N}, G \cap F_{n}$ is relatively open in $F_{n}$. For an $\mathcal{E}$-quasiopen set $G$ and a function $u$ on $G$, $u$ is said to be $\mathcal{E}$-quasicontinuous on $G$ if there exists an $\mathcal{E}$-nest $\left\{F_{n}\right\}$ of closed sets such that $u$ is continuous on each $F_{n} \cap G$, where we may assume that $F_{n} \cap G$ is relatively open in $F_{n}$ for each $n \in \mathbb{N}$. A statement $S(x)$ is said to hold for q.e. $x \in E$ if there exists an exceptional set $N$ such that $\{x \in E \mid S(x)$ does not hold $\} \subset N$.

An increasing sequence $\left\{G_{n}\right\}$ of q.e. finely open Borel sets is called a nest if $\mathbf{P}_{x}\left(\lim _{n \rightarrow \infty} \tau_{G_{n}}=\zeta\right)=1$ for q.e. $x \in E$.

Denote by $\Theta$ the family of nest $\left\{G_{n}\right\}$ of q.e. finely open Borel sets. Note that for an $\mathcal{E}$-nest $\left\{F_{n}\right\}$ of closed sets, $\left\{G_{k}\right\} \in \Theta$ by setting $G_{k}:=F_{k}^{f-i n t}$, $k \in \mathbb{N}$, where $F_{k}^{f \text {-int }}$ means the fine interior of $F_{k}$.

Let $\mathcal{F}_{e}$ be the family of $\mathfrak{m}$-measurable functions $u$ on $E$ such that $|u|<$ $\infty \mathfrak{m}$-a.e. and there exists an $\mathcal{E}$-Cauchy sequence $\left\{u_{n}\right\}$ of $\mathcal{F}$ such that $\lim _{n \rightarrow \infty} u_{n}=u \mathfrak{m}$-a.e. We call $\left\{u_{n}\right\}$ as above an approximating sequence for $u \in \mathcal{F}_{e}$. For any $u, v \in \mathcal{F}_{e}$ and its approximating sequences $\left\{u_{n}\right\},\left\{v_{n}\right\}$, the limit $\mathcal{E}(u, v)=\lim _{n \rightarrow \infty} \mathcal{E}\left(u_{n}, v_{n}\right)$ exists and does not depend on the choices of the approximating sequences for $u, v$. It is known that $\mathcal{E}^{1 / 2}$ on $\mathcal{F}_{e}$ is a seminorm and that $\mathcal{F}=\mathcal{F}_{e} \cap L^{2}(E ; \mathfrak{m})$. We call $\left(\mathcal{E}, \mathcal{F}_{e}\right)$ the extended Dirichlet space of $(\mathcal{E}, \mathcal{F})$. We further let

$$
\begin{aligned}
\dot{\mathcal{F}}_{\text {loc }}:= & \left\{u \in L^{0}(E ; \mathfrak{m}) \mid \text { there exist }\left\{G_{n}\right\} \in \Theta \text { and } u_{n} \in \mathcal{F}\right. \text { such that } \\
& \left.u=u_{n} \mathfrak{m} \text {-a.e. on } G_{n} \text { for each } n \in \mathbb{N}\right\},
\end{aligned}
$$


where $L^{0}(E ; \mathfrak{m})$ denotes the family of all $\mathfrak{m}$-measurable functions. $\dot{\mathcal{F}}_{\text {loc }}$ is called the space of functions locally in $\mathcal{F}$ in the broad sense. It is shown in $\left[11\right.$, Theorem 4.1(i)] that $\mathcal{F} \subset \mathcal{F}_{e} \subset \dot{\mathcal{F}}_{\text {loc }}$ and that every $u \in \dot{\mathcal{F}}_{\text {loc }}$ admits an $\mathcal{E}$ quasicontinuous $\mathfrak{m}$-version $\tilde{u}$. More strongly, every $u \in \mathcal{F}$ admits a strictly $\mathcal{E}$ quasicontinuous $\mathfrak{m}$-version $\tilde{u}$ on $E$ which is defined on $E_{\partial}$ with $\tilde{u}(\partial)=0$. For $u \in \dot{\mathcal{F}}_{\text {loc }}$, we always assume that $\tilde{u}$ is extended to be a real-valued function $\tilde{u}$ on $E_{\partial}$ if not otherwise specified, where we do not necessarily assume that $\tilde{u}(\partial)=0$. However, we can reduce to this case by setting $\tilde{u}-\tilde{u}(\partial)$ on $E_{\partial}$, which is in $\dot{\mathcal{F}}_{\text {loc }}$ as a function defined on $E$. It is proved in [13, Lemma 2.1] that every $u \in \mathcal{F}_{e}$ admits a strictly $\mathcal{E}$-quasicontinuous $\mathfrak{m}$-version $\tilde{u}$ on $E_{\partial}$ with $\tilde{u}(\partial)=0$. Moreover, we know that $\mathcal{F}_{e} \subset \dot{\mathcal{F}}_{\text {loc }}$ by [11, Theorem 4.1(i)]. Except in Lemma 2.2 below, we take $u \in \dot{\mathcal{F}}_{\text {loc }}$ to be represented by its $\mathcal{E}$ quasicontinuous $\mathfrak{m}$-version (when such exists), and we drop the tilde from the notation.

For an $\mathcal{E}$-quasiopen set $G$, we set

$$
\left\{\begin{array}{l}
\mathcal{F}_{G}:=\{u \in \mathcal{F} \mid u=0 \text { q.e. on } E \backslash G\}, \\
\mathcal{E}_{G}(u, v):=\mathcal{E}(u, v) \text { for } u, v \in \mathcal{F}_{G}
\end{array}\right.
$$

$\left(\mathcal{E}_{G}, \mathcal{F}_{G}\right)$ is called the part of $(\mathcal{E}, \mathcal{F})$ on $L^{2}(G ; \mathfrak{m})$. It is known that $\left(\mathcal{E}_{G}, \mathcal{F}_{G}\right)$ is a quasiregular Dirichlet form on $L^{2}(G ; \mathfrak{m})$, and we can always take an $\mathcal{E}_{G^{-}}$ nest $\left\{K_{n}\right\}$ of closed subsets of $G$ consisting of compact sets in $E$. (In general, the quasiregularity of $\left(\mathcal{E}_{G}, \mathcal{F}_{G}\right)$ assures the compactness of $K_{n}$ only with respect to the relative topology on $G$. The assertion is stronger than this.) The space $\left(\mathcal{E}_{G}, \mathcal{F}_{G}\right)$ is associated with the part process on $\mathbf{X}^{G}=\left(\Omega, X_{t}^{G}, \mathbf{P}_{x}\right)$ defined by

$$
X_{t}^{G}(\omega):= \begin{cases}X_{t}(\omega) & t<\tau_{G}(\omega) \\ \partial & t \geq \tau_{G}(\omega)\end{cases}
$$

The following lemmas are used in Section 7.

LEMma 2.1. Let $G$ be an $\mathcal{E}$-quasiopen set, and let $u$ be a function defined on $G$. The following are equivalent:

(1) $u$ is $\mathcal{E}$-quasicontinuous on $G$;

(2) $u$ is $\mathcal{E}_{G}$-quasicontinuous on $G$;

(3) for any open subset $I$ of $\mathbb{R}, u^{-1}(I) \cap G$ is $\mathcal{E}$-quasiopen; and

(4) for any open subset $I$ of $\mathbb{R}, u^{-1}(I) \cap G$ is $\mathcal{E}_{G}$-quasiopen. 
Proof. In general, for a subset $N$ of $G, \mathcal{E}$-polarity of $N$ is equivalent to $\mathcal{E}_{G}$-polarity of $N$, and $N$ is $\mathcal{E}$-quasiopen if and only if it is $\mathcal{E}_{G}$-quasiopen (see [12, Proposition 3.2] and [8, Theorem 4.4.3(ii)]). So the equivalence $(3) \Longleftrightarrow(4)$ is clear.

$(1) \Longleftrightarrow(3)$ : Suppose that $u$ is $\mathcal{E}$-quasicontinuous on $G$. Then there exists an $\mathcal{E}$-nest $\left\{F_{n}\right\}$ of closed sets such that $u$ is continuous on $F_{n} \cap G$ for each $n \in \mathbb{N}$. Take an open set $I$ of $\mathbb{R}$. We see that $u^{-1}(I) \cap G \cap F_{n}$ is open in $F_{n}$ for each $n \in \mathbb{N}$, which implies that the $u^{-1}(I) \cap G$ is $\mathcal{E}$-quasiopen. The proof of the converse is easy. The proof of $(2) \Longleftrightarrow(4)$ is quite similar to that of $(1) \Longleftrightarrow(3)$.

Lemma 2.2. Let $\left(\mathcal{E}, \mathcal{F}_{e}\right)$ be the extended Dirichlet space of $(\mathcal{E}, \mathcal{F})$. Let $G$ be an $\mathcal{E}$-quasiopen set, and $u_{n}$ and $u$ are q.e. finite functions defined on $E$. Assume that all $u_{n} \in \mathcal{F}_{e}$ are $\mathcal{E}$-quasicontinuous on $G$ and that $\left\{u_{n}\right\}$ is $\mathcal{E}$-bounded. Suppose that $u_{n}$ converges to $u$ q.e. as $n \rightarrow \infty$. Then $u$ is $\mathcal{E}$-quasicontinuous on $G$.

Proof. Since $\left\{u_{n}\right\}$ converges to $u$ q.e., we see that $\sup _{n \in \mathbb{N}} u_{n}^{2}<\infty$ q.e. Let $f \in L^{1}(E ; \mathfrak{m})$ be a function satisfying $0<f \leq 1 \mathfrak{m}$-a.e. on $E$. Define a function $g \in L^{1}(E ; \mathfrak{m})$ having $0<g \leq 1 \mathfrak{m}$-a.e. by $g(x):=f /\left(\sup _{n \in \mathbb{N}} u_{n}(x)^{2} \vee\right.$ $1)$. Then we have $\int_{E} u_{n}^{2} g \mathrm{dm} \leq 1$; hence, $\left\{u_{n}\right\} \subset \mathcal{F}_{e}^{g}$ is $\mathcal{E}^{g}$-bounded. Here $\mathcal{F}_{e}^{g}$ is the extended Dirichlet space of $\left(\mathcal{E}^{g}, \mathcal{F}\right)$, where $\mathcal{E}^{g}(u, v):=\mathcal{E}(u, v)+$ $\int_{E} u v g \mathrm{~d} \mathfrak{m}$, for $u, v \in \mathcal{F}$. It is known that $\left(\mathcal{E}^{g}, \mathcal{F}\right)$ is a transient Dirichlet form on $L^{2}(E ; \mathfrak{m})$ and that the $\mathcal{E}^{g}$-quasinotions are equivalent to the $\mathcal{E}$-quasinotions, so we may assume the transience of $(\mathcal{E}, \mathcal{F})$. From the $\mathcal{E}$ boundedness of $\left\{u_{n}\right\}$, taking an adequate subsequence, its Cesàro mean $\mathcal{E}$-converges to $u$, so we may assume that $\left\{u_{n_{k}}\right\} \mathcal{E}$-converges to $u$. By taking a further subsequence $\left\{n_{k}\right\},\left\{u_{n_{k}}\right\}$ converges to $u \mathcal{E}$-quasiuniformly; that is, there exists an $\mathcal{E}$-nest $\left\{F_{l}\right\}$ of closed sets such that $u_{n_{k}}$ uniformly converges to $u$ on each $F_{l}$. Taking a common nest $\left\{F_{l}\right\}$ such that each $\left.u_{n}\right|_{F_{l} \cap G}$ is continuous and $F_{l} \cap G$ is relatively open in $F_{l}$, we see the $\mathcal{E}$-quasicontinuity of $u$ on $G$.

Let $\stackrel{\circ}{\mathcal{M}}$ and $\mathcal{N}_{c}$ denote, respectively, the space of martingale additive functionals $(M A F s)$ of finite energy and the space of continuous additive functionals $(C A F s)$ of zero energy. For $u \in \mathcal{F}_{e}$, the following Fukushima decomposition holds:

$$
u\left(X_{t}\right)-u\left(X_{0}\right)=M_{t}^{u}+N_{t}^{u}
$$


for all $t \in\left[0, \infty\left[\mathbf{P}_{x^{-}}\right.\right.$almost surely $\left(\mathbf{P}_{x^{-}}\right.$a.s. $)$for q.e. $x \in E$, where $M^{u} \in \stackrel{\circ}{\mathcal{M}}$ and $N^{u} \in \mathcal{N}_{c}$.

A positive continuous additive functional $(P C A F)$ of $\mathbf{X}$ (call it $A$ ) determines a measure $\nu=\nu_{A}$ on the Borel subsets of $E$ via the formula

$$
\nu(f)=\uparrow \lim _{t \rightarrow 0} \frac{1}{t} \mathbf{E}_{\mathfrak{m}}\left[\int_{0}^{t} f\left(X_{s}\right) \mathrm{d} A_{s}\right],
$$

in which $f: E \rightarrow[0, \infty]$ is Borel measurable. The measure $\nu$ is necessarily smooth (denoted by $\nu \in S$ ) in the sense that $\nu$ charges no exceptional set of $E$ and there is an $\mathcal{E}$-nest $\left\{F_{n}\right\}$ of closed subsets of $E$ such that $\nu\left(F_{n}\right)<\infty$ for each $n \in \mathbb{N}$. Conversely, given a smooth measure $\nu$, there is a unique PCAF $A^{\nu}$ such that (2.7) holds with $A=A^{\nu}$. We refer to this bijection between smooth measures and PCAFs as the Revuz correspondence, and we refer to $\nu$ as the Revuz measure of $A^{\nu}$. It is proved in [13, Lemma 2.2] that $\mu \in S$ if and only if $\mu$ charges no exceptional set and that there exists $\left\{G_{n}\right\} \in \Theta$ such that $\mu\left(G_{n}\right)<\infty$ for each $n \in \mathbb{N}$.

Take $M, N \in \stackrel{\circ}{\mathcal{M}}$, and denote by $\langle M, N\rangle$ its quadratic covariational process, which is a CAF of bounded variation, and let $\mu_{\langle M, N\rangle}$ be its Revuz measure. For $f \in L^{2}\left(E ; \mu_{\langle M\rangle}\right)$, there exists a unique $f * M \in \stackrel{\circ}{\mathcal{M}}$ such that

$$
\mathbf{e}(f * M, N)=\frac{1}{2} \int_{E} f(x) \mu_{\langle M, N\rangle}(\mathrm{d} x) \quad \text { for } N \in \stackrel{\circ}{\mathcal{M}}
$$

Let $\left(N(x, \mathrm{~d} y), H_{t}\right)$ be a Lévy system for $\mathbf{X}$; that is, $N(x, \mathrm{~d} y)$ is a kernel on $\left(E_{\partial}, \mathscr{B}\left(E_{\partial}\right)\right)$, and $H_{t}$ is a PCAF with bounded 1-potential such that, for any nonnegative Borel function $\phi$ on $E_{\partial} \times E_{\partial}$ vanishing on the diagonal and any $x \in E_{\partial}$,

$$
\mathbf{E}_{x}\left[\sum_{s \leq t} \phi\left(X_{s-}, X_{s}\right)\right]=\mathbf{E}_{x}\left[\int_{0}^{t} \int_{E_{\partial}} \phi\left(X_{s}, y\right) N\left(X_{s}, \mathrm{~d} y\right) \mathrm{d} H_{s}\right] .
$$

To simplify notation, we will write

$$
N \phi(x):=\int_{E_{\partial}} \phi(x, y) N(x, \mathrm{~d} y) .
$$

Let $\mu_{H}$ be the Revuz measure of the PCAF $H$. Then the jumping measure $J$ and the killing measure $\kappa$ of $\mathbf{X}$ are given by

$$
J(\mathrm{~d} x \mathrm{~d} y)=\frac{1}{2} N(x, \mathrm{~d} y) \mu_{H}(\mathrm{~d} x) \quad \text { and } \quad \kappa(\mathrm{d} x)=N(x,\{\partial\}) \mu_{H}(\mathrm{~d} x) .
$$


These measures feature in the Beurling-Deny decomposition of $\mathcal{E}$ : for $f, g \in$ $\mathcal{F}_{e}$

$$
\begin{aligned}
\mathcal{E}(f, g)= & \mathcal{E}^{c}(f, g)+\int_{E \times E}(f(x)-f(y))(g(x)-g(y)) J(\mathrm{~d} x \mathrm{~d} y) \\
& +\int_{E} f(x) g(x) \kappa(\mathrm{d} x),
\end{aligned}
$$

where $\mathcal{E}^{c}$ is the strongly local part of $\mathcal{E}$.

For $u \in \mathcal{F}_{e}$, the martingale part $M_{t}^{u}$ in (2.6) can be decomposed as

$$
M_{t}^{u}=M_{t}^{u, c}+M_{t}^{u, j}+M_{t}^{u, \kappa} \text { for every } t \in[0, \infty[,
$$

$\mathbf{P}_{x^{-}}$a.s. for q.e. $x \in E$, where $M_{t}^{u, c}$ is the continuous part of martingale $M^{u}$, and

$$
\begin{aligned}
M_{t}^{u, j}= & \lim _{\varepsilon \downarrow 0}\left\{\sum_{0<s \leq t}\left(u\left(X_{s}\right)-u\left(X_{s-}\right)\right) \mathbf{1}_{\left\{\left|u\left(X_{s}\right)-u\left(X_{s-}\right)\right|>\varepsilon\right\}} \mathbf{1}_{\{s<\zeta\}}\right. \\
& \left.-\int_{0}^{t}\left(\int_{\left\{y \in E|| u(y)-u\left(X_{s}\right) \mid>\varepsilon\right\}}\left(u(y)-u\left(X_{s}\right)\right) N\left(X_{s}, \mathrm{~d} y\right)\right) \mathrm{d} H_{s}\right\}, \\
M_{t}^{u, \kappa}= & \int_{0}^{t} u\left(X_{s}\right) N\left(X_{s},\{\partial\}\right) \mathrm{d} H_{s}-u\left(X_{\zeta-}\right) \mathbf{1}_{\{t \geq \zeta\}}
\end{aligned}
$$

are the jump and killing parts of $M^{u}$ in $\stackrel{\circ}{\mathcal{M}}$, respectively. The limit in the expression for $M^{u, j}$ is in the sense of convergence in $\stackrel{\circ}{\mathcal{M}}$ and of convergence in probability under $\mathbf{P}_{x}$ for q.e. $x \in E$ for each fixed $t>0$ (see [8, Theorem A.3.9 and p. 341]).

If we let

$$
\begin{aligned}
& \stackrel{\circ}{\mathcal{M}^{c}}:=\{M \in \stackrel{\circ}{\mathcal{M}} \mid M \text { is a continuous MAF }\} \\
& \stackrel{\circ}{\mathcal{M}^{d}}:=\left(\stackrel{\circ}{\mathcal{M}}^{c}\right)^{\perp}=\left\{M \in \stackrel{\circ}{\mathcal{M}} \mid\langle M, N\rangle \equiv 0 \text { for } N \in \stackrel{\circ}{\mathcal{M}}^{c}\right\},
\end{aligned}
$$

then every $M$ has an orthogonal decomposition

$$
M=M^{c}+M^{d}
$$

in the Hilbert space $(\stackrel{\circ}{\mathcal{M}}, \mathbf{e})$. The $\operatorname{MAF} M^{c} \in \stackrel{\circ}{\mathcal{M}}^{c}$ (resp., $M^{d} \in \stackrel{\circ}{\mathcal{M}}^{d}$ ) is nothing but the continuous part (resp., purely discontinuous part) of $M$ discussed 
in [8, Theorem A.3.18]. Moreover, set

$$
\stackrel{\circ}{\mathcal{M}^{j}}:=\left\{M \in \stackrel{\circ}{\mathcal{M}}^{d} \mid\left\langle M, M^{u, \kappa}\right\rangle \equiv 0 \text { for } u \in \mathcal{F}_{e}\right\}, \quad \stackrel{\circ}{\mathcal{M}}^{\kappa}:=\stackrel{\circ}{\mathcal{M}}^{d} \cap\left(\stackrel{\circ}{\mathcal{M}}^{j}\right)^{\perp} .
$$

Then $\stackrel{\circ}{\mathcal{M}}^{j}$ is a closed subspace of $\stackrel{\circ}{\mathcal{M}}$; hence, $M^{d}$ has a unique orthogonal decomposition in $(\stackrel{\circ}{\mathcal{M}}, \mathbf{e})$ as

$$
M^{d}=M^{j}+M^{\kappa}
$$

where $M^{j} \in \stackrel{\circ}{\mathcal{M}}^{j}$ and $M^{\kappa} \in \stackrel{\circ}{\mathcal{M}}^{\kappa}$. For simplicity of notation, we will use the convention $\Delta F_{s}:=F_{s}-F_{s-}$ for any rcll- $\left(\mathscr{F}_{t}\right)$-adapted process $F$. The square bracket $[M, N]$ for $M, N \in \stackrel{\circ}{\mathcal{M}}$ is defined by

$$
[M, N]_{t}:=\left\langle M^{c}, N^{c}\right\rangle_{t}+\sum_{0<s \leq t} \Delta M_{s} \Delta N_{s} .
$$

Then $\langle M, N\rangle$ is the dual predictable projection of $[M, N]$ (see $[8,(\mathrm{~A} .3 .7)]$ ). We further set, for each $i=\emptyset, c, d, j, \kappa$,

$$
\begin{aligned}
\mathcal{M}_{\text {loc }}^{i}:= & \left\{M \mid \text { there exist }\left\{G_{n}\right\} \in \Theta \text { and }\left\{M^{(n)}\right\} \subset \mathcal{M}^{i}\right. \\
& \text { such that } M_{t}=M_{t}^{(n)} \text { for all } t<\tau_{G_{n}} \\
& \text { and } \left.n \in \mathbb{N}, \mathbf{P}_{x} \text {-a.s. for q.e. } x \in E\right\} \\
\mathcal{N}_{c, \text { loc }}:= & \left\{N \mid \text { there exist }\left\{G_{n}\right\} \in \Theta \text { and }\left\{N^{(n)}\right\} \subset \mathcal{N}_{c}\right. \\
& \text { such that } N_{t}=N_{t}^{(n)} \text { for all } t<\tau_{G_{n}} \\
& \text { and } \left.n \in \mathbb{N}, \mathbf{P}_{x} \text {-a.s. for q.e. } x \in E\right\} .
\end{aligned}
$$

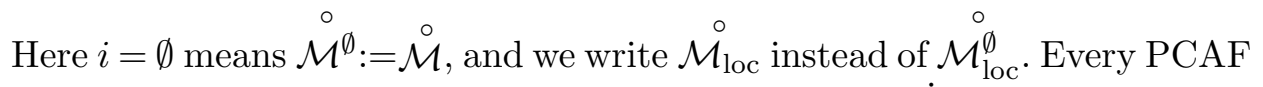
is an element of $\mathcal{N}_{c \text {,loc }}$. It is well known that, for $u \in \dot{\mathcal{F}}_{\text {loc }}$ with $J=\kappa=0$, the Fukushima decomposition (2.6) holds for $t \in\left[0, \zeta\left[\mathbf{P}_{x}\right.\right.$-a.s. for q.e. $x \in E$, where $M^{u} \in \mathcal{M}_{\text {loc }}^{c}$ and $N^{u} \in \mathcal{N}_{c, \text { loc }}$ (see [8, Theorem 5.5.1]).

We introduce the spaces $\stackrel{\circ}{\mathcal{J}}, \mathcal{J}$ of jump functions:

$$
\begin{aligned}
\mathcal{J}:= & \left\{\phi: E_{\partial} \times E_{\partial} \rightarrow \mathbb{R} \mid \phi\right. \text { is a Borel measurable function } \\
& \text { such that } \left.\phi(x, x)=0 \text { for } x \in E_{\partial} \text { and } N\left(\phi^{2}\right) \mu_{H} \in S\right\},
\end{aligned}
$$


and $\stackrel{\circ}{\mathcal{J}}:=\left\{\phi \in \mathcal{J} \mid \int_{E} N\left(\phi^{2}\right) \mathrm{d} \mu_{H}<\infty\right\}$. Further, we set $\mathcal{J}_{\text {as }}:=\{\phi \in \mathcal{J} \mid \widetilde{\phi}=$ $0 J$-a.e. on $E \times E\}, \mathcal{J}_{*}:=\left\{\phi \in \mathcal{J} \mid N\left(\mathbf{1}_{E \times E} \bar{\phi}^{2}\right) \mu_{H} \in S\right\}$, and $\stackrel{\circ}{\mathcal{J}}$ as $:=\stackrel{\circ}{\mathcal{J}} \cap \mathcal{J}_{\text {as }}$. Here $\bar{\phi}(x, y):=\phi(y, x)$ for $x, y \in E_{\partial}$, and $\widetilde{\phi}:=(\phi+\bar{\phi}) / 2$ on $E_{\partial} \times E_{\partial}$. Clearly, $\stackrel{\circ}{\mathcal{J}} \subset \mathcal{J}_{*}$ and $\mathcal{J}_{\text {as }} \subset \mathcal{J}_{*}$, because $\int_{E} N\left(\mathbf{1}_{E \times E} \bar{\phi}^{2}\right) \mathrm{d} \mu_{H}=\int_{E} N\left(\mathbf{1}_{E \times E} \phi^{2}\right) \mathrm{d} \mu_{H}$ for $\phi \in \stackrel{\circ}{\mathcal{J}} \cup \mathcal{J}_{\text {as }}$. Moreover, for $\phi \in \mathcal{J}_{*}$, we see that $\mathbf{1}_{E \times E} \bar{\phi} \in \mathcal{J}_{*}$. For $\phi, \psi \in \mathcal{J}$, we write $\phi \sim \psi$ if $\phi=\psi J^{*}$-a.e. on $E \times E_{\partial}$, where $J^{*}$ is the measure on $E \times E_{\partial}$ defined by $J^{*}(\mathrm{~d} x \mathrm{~d} y):=\frac{1}{2} N(x, \mathrm{~d} y) \mu_{H}(\mathrm{~d} x)$. Then $\sim$ is an equivalence relation, and we denote by $\stackrel{\circ}{\mathcal{J}} / \sim, \mathcal{J} / \sim$ the families of equivalence classes.

Let $\mathcal{M}_{\text {loc }}$ be the space of locally square integrable MAFs, and let $\mathcal{M}_{\text {loc }}^{\llbracket 0, \zeta \llbracket}$ be the space of locally square integrable MAFs on $\llbracket 0, \zeta \llbracket$. That is, $M \in$ $\mathcal{M}_{\text {loc }}$ (resp., $M \in \mathcal{M}_{\text {loc }}^{\llbracket 0, \zeta \llbracket}$ ) if and only if there exists an increasing sequence $\left\{T_{n}\right\}$ (resp., $\left\{S_{n}\right\}$ ) of $\left(\mathscr{F}_{t}\right)$-stopping times and $\left\{M^{(n)}\right\} \subset \mathcal{M}$ such that $\lim _{n \rightarrow \infty} T_{n}=\infty$ (resp., $\lim _{n \rightarrow \infty} S_{n}=\zeta$ ) $\mathbf{P}_{x}$-a.s. for q.e. $x \in E$ and for each $n \in \mathbb{N}, M_{t \wedge T_{n}}=M_{t \wedge T_{n}}^{(n)}$ (resp., $M_{t \wedge S_{n}} \mathbf{1}_{\left\{t \wedge S_{n}<\zeta\right\}}=M_{t \wedge S_{n}}^{(n)} \mathbf{1}_{\left\{t \wedge S_{n}<\zeta\right\}}$ ) for all $t \in$ $\left[0, \infty\left[\mathbf{P}_{x}\right.\right.$-a.s. for q.e. $x \in E$. Let $\mathcal{M}_{\text {loc }}^{c}$ (resp., $\left.\mathcal{M}_{\text {loc }}^{d}\right)$ be the space of locally square integrable continuous (resp., purely discontinuous) MAFs. That is, for $M \in \mathcal{M}_{\text {loc }}^{c}$ (resp., $M \in \mathcal{M}_{\text {loc }}^{d}$ ), we can take $\left\{M^{(n)}\right\}$ from $\mathcal{M}^{c}$ (resp., $\mathcal{M}^{d}$ ) in the above definition. Similarly, we can define the space $\mathcal{M}_{\text {loc }}^{c, \llbracket 0, \zeta \mathbb{I}}$ (resp., $\mathcal{M}_{\text {loc }}^{d, \llbracket 0, \zeta \llbracket}$ ) of locally square integrable continuous (resp., purely discontinu-

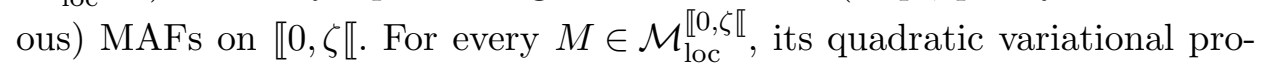
cess $\langle M\rangle$ can be defined to be a PCAF (see [4, Proposition 2.8]), and $M$ is

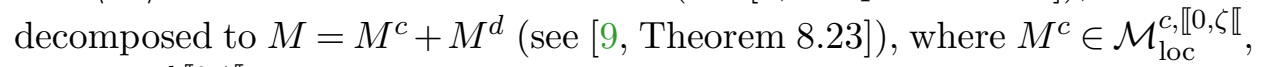
$M^{d} \in \mathcal{M}_{\mathrm{loc}}^{d, \llbracket 0, \zeta \llbracket}$ have the property $\left\langle M^{c}, M^{d}\right\rangle \equiv 0$.

By [13, Lemma 2.1] and [14, Theorem 1.1], there exists a one-to-one correspondence between $\stackrel{\circ}{\mathcal{J}} / \sim$ (resp., $\mathcal{J} / \sim)$ and $\stackrel{\circ}{\mathcal{M}}^{d}$ (resp., $\mathcal{M}_{\text {loc }}^{d, \llbracket 0, \zeta \llbracket}$ ).

We define subclasses of $\mathcal{M}_{\text {loc }}^{d, \llbracket 0, \zeta \llbracket}$ as follows:

$$
\begin{aligned}
& \mathcal{M}_{\mathrm{loc}}^{j, \llbracket 0, \zeta \llbracket}:=\left\{M \in \mathcal{M}_{\mathrm{loc}}^{d, \llbracket 0, \zeta \llbracket} \mid \phi(\cdot, \partial)=0 \text { к-a.e. on } E\right\}, \\
& \mathcal{M}_{\mathrm{loc}}^{\kappa, \llbracket 0, \zeta \llbracket}:=\left\{M \in \mathcal{M}_{\mathrm{loc}}^{d, \llbracket 0, \zeta \llbracket} \mid \phi=0 \quad J \text {-a.e. on } E \times E\right\} \text {. }
\end{aligned}
$$

Then we have that $M \in \mathcal{M}_{\mathrm{loc}}^{j, \llbracket 0, \zeta \llbracket}, N \in \mathcal{M}_{\mathrm{loc}}^{\kappa, \llbracket 0, \zeta \llbracket}$ implies that $\langle M, N\rangle \equiv 0$ $\mathbf{P}_{x}$-a.s. for q.e. $x \in E$ and that every $M \in \mathcal{M}_{\mathrm{loc}}^{\llbracket 0, \zeta \llbracket}$ is decomposed to $M=$ $M^{c}+M^{j}+M^{\kappa}$, where $M^{c} \in \mathcal{M}_{\mathrm{loc}}^{c, \llbracket 0, \zeta \llbracket}, M^{j} \in M_{\mathrm{loc}}^{j, \llbracket 0, \zeta \llbracket}, M^{\kappa} \in \mathcal{M}_{\mathrm{loc}}^{\kappa, \llbracket 0, \zeta \llbracket}$ have 
the properties $\left\langle M^{c}, M^{j}\right\rangle \equiv\left\langle M^{j}, M^{\kappa}\right\rangle \equiv\left\langle M^{\kappa}, M^{c}\right\rangle \equiv 0$ in view of $[14$, Theorem 1.1].

By $\left[14\right.$, Remark 1.1], for each $i=c, d, j, \kappa$ we have $\mathcal{M}_{\mathrm{loc}}^{i, \llbracket 0, \zeta \llbracket}=\stackrel{\circ}{\mathrm{M}}_{\mathrm{loc}}^{i+}(i=$ $c, d, j, \kappa)$, where

$$
\begin{aligned}
\mathcal{M}_{\mathrm{loc}}^{i+}:= & \left\{M \mid \text { there exists }\left\{G_{n}\right\} \in \Theta \text { and } M^{(n)} \in \stackrel{\circ}{\mathcal{M}}^{i}\right. \text { such that } \\
& \left.M_{t}=M_{t}^{(n)} \text { for all } t \leq \tau_{G_{n}} \text { and } n \in \mathbb{N}, \mathbf{P}_{x^{-}} \text {a.s. for q.e. } x \in E\right\} .
\end{aligned}
$$

More strongly, we have $\mathcal{M}_{\text {loc }}^{c, \llbracket 0, \zeta \llbracket}=\mathcal{M}_{\text {loc }}^{c}$.

We introduce a subclass $\dot{\mathcal{F}}_{\text {loc }}^{\dagger}$ of $\dot{\mathcal{F}}_{\text {loc }}$ as follows:

$$
\dot{\mathcal{F}}_{\text {loc }}^{\dagger}:=\left\{u \in \dot{\mathcal{F}}_{\text {loc }} \mid N\left(\mathbf{1}_{E \times E}(u(\cdot)-u)^{2}\right) \mu_{H} \in S\right\} .
$$

Note that $u \in \dot{\mathcal{F}}_{\text {loc }}^{\dagger}$ if and only if $u \in \dot{\mathcal{F}}_{\text {loc }}$ and $(u(\cdot)-u)^{2} \in \mathcal{J}$. Clearly, $\dot{\mathcal{F}}_{\text {loc }}^{\dagger}$ is a linear subspace of $\dot{\mathcal{F}}_{\text {loc }}$, and $\mathbf{1}_{E_{\partial}}, \mathbf{1}_{E} \in \dot{\mathcal{F}}_{\text {loc }}^{\dagger}$. By [4, Remark 3.9] and the fact that $\kappa \in S$, we see that $\mathcal{F}_{e} \cup\left(\dot{\mathcal{F}}_{\text {loc }}\right)_{b} \subset \dot{\mathcal{F}}_{\text {loc }}^{\dagger}$. For $u, v \in \dot{\mathcal{F}}_{\text {loc }}^{\dagger}$, we see that $u v \in \dot{\mathcal{F}}_{\text {loc }}^{\dagger}$, provided that $u$ or $v$ is bounded. From in [14, Theorem 1.1], for $u \in \dot{\mathcal{F}}_{\text {loc }}^{\dagger}$ with $(u(\cdot)-u)^{2} \in \stackrel{\circ}{\mathcal{J}}$ (resp., $u \in \dot{\mathcal{F}}_{\text {loc }}^{\dagger}$ ), there exists an $M^{u, d} \in \mathcal{M}^{d}$ (resp., $M^{u, d} \in \mathcal{M}_{\mathrm{loc}}^{d, \llbracket 0, \zeta \llbracket}$ ) such that $\Delta M_{t}^{u, d}=\Delta u\left(X_{t}\right)$ for all $t \in[0, \infty[$ (resp., $\left[0, \zeta[) \mathbf{P}_{x}\right.$-a.s. for q.e. $x \in E$. Moreover, we define

$$
\begin{aligned}
\mathcal{F}_{\text {loc }}^{\dagger}:= & \left\{u \in \mathcal{F}_{\text {loc }} \mid \text { for any compact set } K,\right. \\
& \left.\int_{K \times E}(u(y)-u(x))^{2} J(\mathrm{~d} x \mathrm{~d} y)<\infty\right\} .
\end{aligned}
$$

Here $\mathcal{F}_{\text {loc }}$ is the space of functions locally in $\mathcal{F}$ in the ordinary sense (see [8]). Clearly, $\mathcal{F}_{\text {loc }}^{\dagger} \subset \dot{\mathcal{F}}_{\text {loc }}^{\dagger}$. For $u \in \mathcal{F}_{\text {loc }}, u \in \mathcal{F}_{\text {loc }}^{\dagger}$ if and only if, for any compact set $K$ with its relatively compact open neighborhood $G$, it holds that

$$
\int_{K \times G^{c}}(u(y)-u(x))^{2} J(\mathrm{~d} x \mathrm{~d} y)<\infty .
$$

We see that $\mathcal{F} \cup\left(\mathcal{F}_{\text {loc }}\right)_{b} \subset \mathcal{F}_{\text {loc }}^{\dagger}$, because of $J\left(K \times G^{c}\right)<\infty$ (see [11, Corollary 5.1]), where $K$ and $G$ are noted as above.

REMARK 2.1. In [13] we introduced the following classes:

$$
\dot{\mathcal{F}}_{\text {loc }}^{\ddagger}:=\left\{u \in \dot{\mathcal{F}}_{\text {loc }}^{\dagger} \mid u(\partial) \in \mathbb{R} \text { and }(u(\cdot)-u(\partial))^{2} \kappa \in S\right\},
$$




$$
\begin{aligned}
\mathcal{F}_{\text {loc }}^{\ddagger}:= & \left\{u \in \mathcal{F}_{\text {loc }}^{\dagger} \mid u(\partial) \in \mathbb{R} \text { and for any compact set } K,\right. \\
& \left.\int_{K}(u(x)-u(\partial))^{2} \kappa(\mathrm{d} x)<\infty\right\} .
\end{aligned}
$$

These classes are unnecessary. Indeed, we can easily see that any $u \in \dot{\mathcal{F}}_{\text {loc }}^{\dagger}$ (resp., $u \in \mathcal{F}_{\text {loc }}^{\dagger}$ ) with $u(\partial) \in \mathbb{R}$ satisfies $(u(\cdot)-u(\partial))^{2} \kappa \in S$ (resp., $\int_{K}(u(x)-$ $u(\partial))^{2} \kappa(\mathrm{d} x)<\infty$ for any compact set $\left.K\right)$.

\section{$\S 3 . \quad$ Nakao integrals}

We summarize the extension of the Nakao operator and Nakao integrals investigated in [13] with corrections.

Let $\mathcal{N}_{c}^{*} \subset \mathcal{N}_{c}$ denote the class of continuous additive functionals of the form $N^{u}+\int_{0}^{*} g\left(X_{s}\right) \mathrm{d} s$ for some $u \in \mathcal{F}$ and $g \in L^{2}(E ; \mathfrak{m})$. Nakao [18] constructed a linear operator $\Gamma$ from $\stackrel{\circ}{\mathcal{M}}$ into $\mathcal{N}_{c}^{*}$ in the following way. For every $Z \in \stackrel{\circ}{\mathcal{M}}$, there is a unique $w \in \mathcal{F}$ such that

$$
\mathcal{E}_{1}(w, f)=\frac{1}{2} \mu_{\left\langle M^{f}+M^{f, \kappa}, Z\right\rangle}(E) \quad \text { for every } f \in \mathcal{F} .
$$

This unique $w$ is denoted by $\gamma(Z)$. The operator $\Gamma$ is defined by

$$
\Gamma(Z)_{t}:=N_{t}^{\gamma(Z)}-\int_{0}^{t} \gamma(Z)\left(X_{s}\right) \mathrm{d} s \quad \text { for } Z \in \stackrel{\circ}{\mathcal{M}}
$$

It is shown in Nakao [18, (3.6)] that $\Gamma(Z)$ can be characterized by the following equation:

$$
\lim _{t \downarrow 0} \frac{1}{t} \mathbf{E}_{g \mathfrak{m}}\left[\Gamma(Z)_{t}\right]=-\frac{1}{2} \mu_{\left\langle M^{g}+M^{g, \kappa}, Z\right\rangle}(E) \quad \text { for every } g \in \mathcal{F}_{b} .
$$

Here $\mathcal{F}_{b}:=\mathcal{F} \cap L^{\infty}(E ; \mathfrak{m})$. So, in particular, we have $\Gamma\left(M^{u}\right)=N^{u}$ for $u \in \mathcal{F}$. By [13, Lemma 3.1], we have $\Gamma\left(M^{u}\right)=N^{u}$ for $u \in \mathcal{F}_{e}$.

In the same way as Nakao [18] (see [4, (3.13)]), we can define a stochastic integral by using the operator $\Gamma$, as follows. For $M \in \stackrel{\circ}{\mathcal{M}}$ with its jump function $\varphi \in \stackrel{\circ}{\mathcal{J}}$ and $f \in \mathcal{F}_{e} \cap L^{2}(E ; \mu\langle M\rangle)$, we set

$$
\begin{aligned}
& \int_{0}^{t} f\left(X_{s}\right) \mathrm{d} \Gamma(M)_{s} \\
& \quad:=\Gamma(f * M)_{t}-\frac{1}{2}\left\langle M^{f, c}+M^{f, j}, M^{c}+M^{j}+K\right\rangle_{t}, \quad t \in[0, \infty[,
\end{aligned}
$$


where $(f * M)_{t}:=\int_{0}^{t} f\left(X_{s-}\right) \mathrm{d} M_{s}$ and $K \in \stackrel{\circ}{\mathcal{M}}^{d}$ with $K_{t}-K_{t-}=-\mathbf{1}_{E \times E}(\varphi+$ $\left.\bar{\varphi})\left(X_{t-}, X_{t}\right) t \in\right] 0, \infty\left[\mathbf{P}_{x}\right.$-a.s. for q.e. $x \in E$. The integral (3.4) is well defined under $\mathbf{P}_{x}$ for q.e. $x \in E$. In this paper we call the operator $\Gamma$ the Nakao operator and the integral (3.4) the Nakao integral.

For any $M \in \mathcal{M}_{\mathrm{loc}}^{c, \llbracket 0, \zeta \llbracket}$ (in particular, for $M \in \mathcal{M}_{\mathrm{loc}}^{c}$ ), $\Gamma(M)$ can be defined as an element in $\mathcal{N}_{c, \text { loc }}$ in view of $[13$, Lemma 3.2$]$. The space $\left(\mathcal{M}_{\text {loc }}^{d, \llbracket 0, \zeta \llbracket}\right)_{*}$ (resp., $\left.\left(\mathcal{M}_{\mathrm{loc}}^{d, \llbracket 0, \zeta \llbracket}\right)_{\text {as }}\right)$ is defined to be a subclass of $\mathcal{M}_{\mathrm{loc}}^{d, \llbracket 0, \zeta \llbracket}$ associated to $\mathcal{J}_{*} / \sim$ (resp., $\mathcal{J}_{\text {as }} / \sim$ ) by $[14$, Theorem 1.1].

We say that $M \in\left(\mathcal{M}_{\mathrm{loc}}^{\llbracket 0, \zeta \llbracket}\right)_{*}$ (resp., $\left.M \in\left(\mathcal{M}_{\mathrm{loc}}^{\llbracket 0, \zeta \llbracket}\right)_{\text {as }}\right)$ if and only if its purely discontinuous part $M^{d}$ belongs to $\left(\mathcal{M}_{\mathrm{loc}}^{d, \llbracket 0, \zeta \llbracket}\right)_{*}\left(\operatorname{resp} .,\left(\mathcal{M}_{\mathrm{loc}}^{d, \llbracket 0, \zeta \llbracket}\right)_{\mathrm{as}}\right)$. For $M \in\left(\mathcal{M}_{\text {loc }}^{\llbracket 0, \zeta \llbracket}\right)_{*}$ with its jump function $\varphi \in \mathcal{J}_{*}$, let $M^{c} \in \mathcal{M}_{\text {loc }}^{c, \llbracket 0, \zeta \llbracket}$ be its continuous part; take $M^{j} \in \mathcal{M}_{\text {loc }}^{j, \llbracket 0, \zeta \llbracket}$ associated with $\mathbf{1}_{E \times E} \varphi \in \mathcal{J}$, and take $K \in \mathcal{M}_{\mathrm{loc}}^{j, \llbracket 0, \zeta \llbracket}$ associated with $-\mathbf{1}_{E \times E}(\varphi+\bar{\varphi}) \in \mathcal{J}$.

We shall extend $\Gamma$ over $\left(\mathcal{M}_{\mathrm{loc}}^{\llbracket 0, \zeta \llbracket}\right)_{*}$ and establish (3.4) for more general integrands and integrators under $\mathbf{P}_{x}$ for q.e. $x \in E$. By [13, Lemma 3.3], for $M \in\left(\mathcal{M}_{\mathrm{loc}}^{\llbracket 0, \zeta \llbracket}\right)_{*}$ with its jump function $\varphi \in \mathcal{J}_{*}$ and a q.e. finely open Borel set $G$ satisfying $\mathbf{1}_{G \times E} \varphi, \mathbf{1}_{G \times E} \bar{\varphi} \in \stackrel{\circ}{\mathcal{J}}$, and $f \in \mathcal{F}_{e} \cap L^{2}\left(E ; \mu_{\langle M\rangle}\right)$ satisfying $f=0$ m-a.e. on $G$, we have $\Gamma(f * M)_{t}=\frac{1}{2}\left\langle M^{f, c}+M^{f, j}, M^{c}+M^{j}+K\right\rangle_{t}$ for $t \in\left[0, \tau_{G}\left[\mathbf{P}_{x^{-}}\right.\right.$a.s. for q.e. $x \in E$, which ensures the well-definedness in the following definition.

Definition 3.1 (extended Nakao operators and Nakao integrals). We consider $M \in\left(\mathcal{M}_{\text {loc }}^{\llbracket 0, \zeta \llbracket}\right)_{*}$ with its jump function $\varphi \in \mathcal{J}_{*}$ and $f \in \dot{\mathcal{F}}_{\text {loc }}$. Let $\left\{G_{k}\right\} \in \Theta$ be a common nest such that $\mu_{\langle M\rangle}\left(G_{k}\right)<\infty, f=f_{k}$ m-a.e. on $G_{k}$ for some $f_{k} \in \mathcal{F}_{b}$, and $\mathbf{1}_{G_{k} \times E} \varphi, \mathbf{1}_{G_{k} \times E} \bar{\varphi} \in \stackrel{\circ}{\mathcal{J}}$ for each $k \in \mathbb{N}$. Set $E_{k}:=\{x \in$ $\left.E \mid \mathbf{E}_{x}\left[\int_{0}^{\tau_{G}} e^{-t} g\left(X_{t}\right) \mathrm{d} t\right]>1 / k\right\}$ for $g \in L^{2}(E ; \mathfrak{m})$ with $0<g \leq 1 \mathfrak{m}$-a.e. Then $e_{k}:=k \mathbf{E} \cdot\left[\int_{0}^{\tau_{G_{k}}} e^{-t} g\left(X_{t}\right) \mathrm{d} t\right] \wedge 1 \in \mathcal{F}_{G_{k}}$ satisfies $\mathbf{1}_{E_{k}} \leq e_{k} \leq \mathbf{1}_{G_{k}}$ q.e. on $E$. In view of [11, Lemma 3.3], we have $\left\{E_{k}\right\} \in \Theta$. Then we set

$$
\Gamma(M)_{t}:=\Gamma\left(e_{k} * M\right)_{t}-\frac{1}{2}\left\langle M^{e_{k}, c}+M^{e_{k}, j}, M^{c}+M^{j}+K\right\rangle_{t} \quad \text { for } t \in\left[0, \tau_{E_{k}}[\right.
$$

for each $k \in \mathbb{N}, \mathbf{P}_{x^{-}}$a.s. for q.e. $x \in E$. For $M \in\left(\mathcal{M}_{\mathrm{loc}}^{\llbracket 0, \zeta \llbracket}\right)_{*}$ and $f \in \mathcal{F}_{e} \cap$ $L^{2}\left(E ; \mu_{\langle M\rangle}\right)$, we set 


$$
\begin{aligned}
& \int_{0}^{t} f\left(X_{s}\right) \mathrm{d} \Gamma(M)_{s} \\
& \quad:=\Gamma(f * M)_{t}-\frac{1}{2}\left\langle M^{f, c}+M^{f, j}, M^{c}+M^{j}+K\right\rangle_{t} \quad \text { for } t \in[0, \infty[
\end{aligned}
$$

$\mathbf{P}_{x}$-a.s. for q.e. $x \in E$. For general $f \in \dot{\mathcal{F}}_{\text {loc }}$ and $M \in\left(\mathcal{M}_{\text {loc }}^{\llbracket 0, \zeta \llbracket}\right)_{*}$ with $e_{k}$ and $\left\{E_{k}\right\}$ as noted above, we set

$$
\int_{0}^{t} f\left(X_{s}\right) \mathrm{d} \Gamma(M)_{s}:=\int_{0}^{t}\left(f e_{k}\right)\left(X_{s}\right) \mathrm{d} \Gamma(M)_{s} \quad \text { for } t \in\left[0, \tau_{E_{k}}[\right.
$$

for each $k \in \mathbb{N} \mathbf{P}_{x^{-a}}$ a.s. for q.e. $x \in E$. Note that $f e_{k} \in \mathcal{F}_{b} \cap L^{2}\left(E ; \mu_{\langle M\rangle}\right)$ for each $k \in \mathbb{N}$. We call $\Gamma$ defined over $\left(\mathcal{M}_{\text {loc }}^{\llbracket 0, \zeta \llbracket}\right)_{*}$ an extended Nakao operator, and we call the integral in the left-hand side of (3.5) an extended Nakao integral. These are well defined for all $t \in\left[0, \zeta\left[\mathbf{P}_{x^{-}}\right.\right.$a.s. for q.e. $x \in E$ in view of [13, Lemma 3.3] as noted above and are elements in $\mathcal{N}_{c, \text { loc }}$.

For $f \in \dot{\mathcal{F}}_{\text {loc }}$ and $M \in\left(\mathcal{M}_{\mathrm{loc}}^{\llbracket 0, \zeta \llbracket}\right)_{*}$, we see that

$$
\int_{0}^{t} f\left(X_{s}\right) \mathrm{d} \Gamma\left(M^{c}\right)_{s}=\Gamma\left(f * M^{c}\right)_{t}-\frac{1}{2}\left\langle M^{f, c}, M^{c}\right\rangle_{t}
$$

for all $t \in\left[0, \zeta\left[\mathbf{P}_{x}\right.\right.$-a.s. for q.e. $x \in E$, where $\Gamma\left(f * M^{c}\right)_{t}$ can be defined by way of [13, Lemma 3.2]. Moreover, from [13, Corollary 4.1], we have that

$$
\int_{0}^{t} f\left(X_{s}\right) \mathrm{d} \Gamma(K)_{s}=0
$$

holds for all $t \in\left[0, \zeta\left[\mathbf{P}_{x}\right.\right.$-a.s. for q.e. $x \in E$. In particular, $\Gamma(K)_{t}=0$ for all $t \in\left[0, \zeta\left[\mathbf{P}_{x}\right.\right.$-a.s. for q.e. $x \in E$. Here $K \in\left(\mathcal{M}_{\text {loc }}^{\llbracket 0, \zeta \llbracket}\right)_{*}$ is the purely discontinuous local MAF associated to $-\mathbf{1}_{E \times E}(\varphi+\bar{\varphi}) \in \mathcal{J}$.

\section{$\S 4$. Progressively additive functionals in the strong sense}

In this section, we shall refine the notion of additive functionals called progressively additive functionals in the strong sense. We begin with some details on the completion of filtrations. Let $\mathscr{P}(E)$ be the family of all probability measures on $E$. For each $\nu \in \mathscr{P}(E)$, let $\mathscr{F}_{\infty}^{\nu}$ (resp., $\mathscr{F}_{t}^{\nu}$ ) be the $\mathbf{P}_{\nu}$-completion of $\mathscr{F}_{\infty}^{0}$ (resp., $\mathbf{P}_{\nu^{-}}$-completion of $\mathscr{F}_{t}^{0}$ in $\mathscr{F}_{\infty}^{\nu}$ ), and set $\mathscr{F}_{\infty}:=\bigcap_{\nu \in \mathscr{P}(E)} \mathscr{F}_{\infty}^{\nu}$ and $\mathscr{F}_{t}:=\bigcap_{\nu \in \mathscr{P}(E)} \mathscr{F}_{t}^{\nu}$. Further, we introduce $\mathscr{F}_{\infty}^{*}:=$ $\bigcap_{\nu \in S_{00}, \nu \neq 0} \mathscr{F}_{\infty}^{\widehat{\nu}}$ and $\mathscr{F}_{t}^{*}:=\bigcap_{\nu \in S_{00}, \nu \neq 0} \mathscr{F}_{t}^{\widehat{\nu}}$, where $S_{00}:=\left\{\mu \in S_{0} \mid \mu(E)<\right.$ 
$\infty$ and $\left.U_{1} \mu \in L^{\infty}(E ; \mathfrak{m})\right\}$ and $\widehat{\nu}:=\nu / \nu(E)$. Here $S_{0}$ denotes the family of measures of finite energy, and $U_{1} \mu$ denotes its 1-potential (see [8]). Clearly, $\mathscr{F}_{\infty} \subset \mathscr{F}_{\infty}^{*}$ and $\mathscr{F}_{t} \subset \mathscr{F}_{t}^{*}$ for each $t \in[0, \infty[$.

First we recall the definition of additive functionals.

Definition 4.1 (additive functional). An $\left(\mathscr{F}_{t}\right)$-adapted (resp., $\left(\mathscr{F}_{t}^{*}\right)$ adapted) process $A=\left(A_{t}\right)_{t \geq 0}$ with values in $[-\infty, \infty]$ is said to be an additive functional (resp., relaxed additive functional $\left(\mathrm{AF}^{*}\right)$ ) if there exist a defining set $\Xi \in \mathscr{F}_{\infty}$ (resp., $\Xi \in \mathscr{F}_{\infty}^{*}$ ) and an exceptional set $N$ satisfying the following conditions:

(1) $\mathbf{P}_{x}(\Xi)=1$ for all $x \in E \backslash N$;

(2) $\theta_{t} \Xi \subset \Xi$ for all $t \geq 0$; in particular, $\omega_{\partial} \in \Xi$ and $\mathbf{P}_{\partial}(\Xi)=1$, because of $\omega_{\partial}=\theta_{\zeta(\omega)}(\omega)$ for all $\omega \in \Xi$

(3) for all $\omega \in \Xi, A$. ( $\omega)$ is right-continuous and has a left limit on $[0, \zeta(\omega)[$, $A_{0}(\omega)=0,\left|A_{t}(\omega)\right|<\infty$ for $t<\zeta(\omega)$, and $A_{t+s}(\omega)=A_{t}(\omega)+A_{s}\left(\theta_{t} \omega\right)$ for all $t, s \geq 0$; and

(4) for all $t \geq 0, A_{t}\left(\omega_{\partial}\right)=0$; in particular, under the additivity in (3), $A_{t}(\omega)=A_{\zeta(\omega)}(\omega)$ for all $t \geq \zeta(\omega)$ and $\omega \in \Xi$.

An additive functional $A$ is called right-continuous with left limits (rcll-AF)

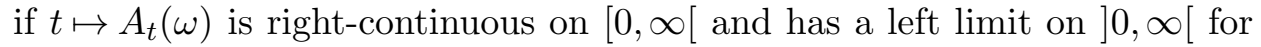
each $\omega \in \Xi$. An additive functional $A$ is said to be a finite (resp., continuous additive functional $(C A F))$ if $\left|A_{t}(\omega)\right|<\infty$, for all $t \in\left[0, \infty\left[\right.\right.$ (resp., $t \mapsto A_{t}(\omega)$ is continuous on $[0, \infty[)$ for each $\omega \in \Xi$. A $[0, \infty]$-valued CAF is called a positive continuous additive functional $(P C A F)$. Two additive functionals $A$ and $B$ are called equivalent if there exist a common defining set $\Xi \in \mathscr{F}_{\infty}$ and an exceptional set $N$ such that $A_{t}(\omega)=B_{t}(\omega)$ for all $t \in[0, \infty[$ and $\omega \in \Xi$. We call $A=\left(A_{t}\right)_{t \geq 0}$ an additive functional on $\llbracket 0, \zeta \llbracket$ or a local additive functional if $A$ is $\left(\mathscr{F}_{t}\right.$ )-adapted and satisfies (1), (2), (4), and the property $\left(3^{\prime}\right)$ in which (3) is modified so that the additivity condition is required only for $t+s<\zeta(\omega)$. The notions of rcll-AF, CAF, and PCAF on $\llbracket 0, \zeta \llbracket$ are similarly defined. Two additive functionals on $\llbracket 0, \zeta \llbracket, A$ and $B$, are called equivalent if there exist a common defining set $\Xi \in \mathscr{F}_{\infty}$ and an exceptional set $N$ such that $A_{t}(\omega)=B_{t}(\omega)$ for all $t \in[0, \zeta[$ and $\omega \in \Xi$. These notions can be formulated for $\mathrm{AF}^{*}$ by replacing $\Xi \in \mathscr{F}_{\infty}$ with $\Xi \in \mathscr{F}_{\infty}^{*}$.

Definition 4.2 (multiplicative functional). An $\left(\mathscr{F}_{t}\right)$-adapted (resp., $\left(\mathscr{F}_{t}^{*}\right)$-adapted) process $M=\left(M_{t}\right)_{t \geq 0}$ with values in $[0, \infty]$ is said to be a multiplicative functional $(M F)$ (resp., relaxed multiplicative functional $\left(M F^{*}\right)$ ) 
if there exist a defining set $\Xi \in \mathscr{F}_{\infty}$ (resp., $\Xi \in \mathscr{F}_{\infty}^{*}$ ) and an exceptional set $N$ satisfying conditions (1) and (2) in Definition 4.1 and

(3) for all $\omega \in \Xi, M .(\omega)$ is right-continuous and has a left limit on $[0, \zeta(\omega)[$, $M_{0}(\omega)=1$ and $M_{t+s}(\omega)=M_{t}(\omega) M_{s}\left(\theta_{t} \omega\right)$ for all $t, s \geq 0$; and

(4) for all $t \geq 0, M_{t}\left(\omega_{\partial}\right)=1$; in particular, under the multiplicativity in (3), $M_{t}(\omega)=M_{\zeta(\omega)}(\omega)$ for all $t \geq \zeta(\omega)$ and $\omega \in \Xi$.

An MF on $\llbracket 0, \zeta \llbracket$ is similarly defined.

Definition 4.3. For any $t>0$, we say that two sample paths $\omega$ and $\omega^{\prime}$ are t-equivalent if $\omega(s)=\omega^{\prime}(s)$ for all $s \in[0, t]$. We say that two sample paths $\omega$ and $\omega^{\prime}$ are pre-t-equivalent if $\omega(s)=\omega^{\prime}(s)$ for all $s \in[0, t[$.

The following lemma is proved in [4, Lemma 2.15]. Recall that $\left\{\theta_{t}, t>0\right\}$ denotes the time-shift operators on the path space for the process $\mathbf{X}$.

Lemma 4.1 (see [4, Lemma 2.15]). For $t, s>0$,

(1) $\theta_{t} r_{t+s} \omega$ is $s$-equivalent to $r_{s} \omega$ if $t+s<\zeta(\omega)$ or $s \geq \zeta(\omega)$; and

(2) $r_{t} \theta_{s} \omega$ is pre-t-equivalent to $r_{t+s} \omega$; moreover, if $\omega$ is continuous at $s$, then $r_{t} \theta_{s} \omega$ is t-equivalent to $r_{t+s} \omega$.

For an rcll-AF (or rcll-AF* $A_{t}$ of $\mathbf{X}$ adapted to $\left(\mathscr{F}_{t}^{0}\right)_{t \geq 0}, A_{t}(\omega)=A_{t}\left(\omega^{\prime}\right)$ if $\omega$ and $\omega^{\prime}$ are $t$-equivalent, and $A_{t-}(\omega)=A_{t-}\left(\omega^{\prime}\right)$ if $\omega$ and $\omega^{\prime}$ are pre-tequivalent. If the measurability is lifted, it does not necessarily hold. In [4, Definition 2.1], we introduced the following notion of progressively additive functional in order to justify the stochastic calculus discussed in [4].

Definition 4.4 (PrAF). A process $A=\left(A_{t}\right)_{t \geq 0}$ with values in $\overline{\mathbb{R}}:=$ $[-\infty, \infty]$ is said to be a progressively additive functional $(\operatorname{Pr} A F)$ (resp., relaxed progressively additive functional $\left.\left(\operatorname{Pr} A F^{*}\right)\right)$ if $A$ is $\left(\mathscr{F}_{t}\right)$-adapted (resp., $\left(\mathscr{F}_{t}^{*}\right)$-adapted) and there exist defining sets $\Xi \in \mathscr{F}_{\infty}, \Xi_{t} \in \mathscr{F}_{t}$ (resp., $\left.\Xi \in \mathscr{F}_{\infty}^{*}, \Xi_{t} \in \mathscr{F}_{t}^{*}\right)$ for each $t>0$ and an exceptional set $N$ satisfying the conditions

(1) $\mathbf{P}_{x}(\Xi)=1$ for all $x \in E \backslash N$, and $\Xi \subset \Xi_{t} \subset \Xi_{s}$ for every $t>s>0$, and $\Xi=\bigcap_{t>0} \Xi_{t}$

(2) $\theta_{s} \Xi \subset \Xi$ for all $s \geq 0$ and $\theta_{s}\left(\Xi_{t}\right) \subset \Xi_{t-s}$ for all $\left.s \in\right] 0, t[$; in particular, $\omega_{\partial} \in \Xi \subset \Xi_{t}$ and $\mathbf{P}_{\partial}(\Xi)=\mathbf{P}_{\partial}\left(\Xi_{t}\right)=1$ under (1);

(3) for all $\omega \in \Xi_{t}, A$.( $\left.\omega\right)$ is defined on $[0, t[$, and it is right-continuous on $[0, t \wedge \zeta(\omega)[$ and has a left limit on $] 0, t] \cap] 0, \zeta(\omega)\left[\right.$ such that $A_{0}(\omega)=0$, $\left|A_{s}(\omega)\right|<\infty$ for $s \in\left[0, t \wedge \zeta(\omega)\left[\right.\right.$, and $A_{p+q}(\omega)=A_{p}(\omega)+A_{q}\left(\theta_{p} \omega\right)$ for all $p, q \geq 0$ with $p+q<t$ 
(4) for all $t \geq 0, A_{t}\left(\omega_{\partial}\right)=0$; and

(5) for any $t>0$ and pre-t-equivalent paths $\omega, \omega^{\prime} \in \Omega, \omega \in \Xi_{t}$ implies that $\omega^{\prime} \in \Xi_{t}, A_{s}(\omega)=A_{s}\left(\omega^{\prime}\right)$ for any $s \in[0, t[$.

Furthermore, $A$ is called an $\operatorname{rcll}-\operatorname{Pr} A F$ if, for each $t>0$ and $\omega \in \Xi_{t}, s \mapsto$ $A_{s}(\omega)$ is right-continuous on $[0, t[$ and has left limits on $] 0, t]$, and a $\operatorname{PrAF}$ is said to be finite (resp., continuous) if $\left|A_{s}(\omega)\right|<\infty$, for all $s \in[0, t[$ (resp., continuous on $\left[0, t[)\right.$ for every $\omega \in \Xi_{t}$.

An $\overline{\mathbb{R}}$-valued process $A$ is called a $\operatorname{Pr} A F$ on $\llbracket 0, \zeta \llbracket$ if $A$ is $\left(\mathscr{F}_{t}\right)$-adapted, and there exist $\Xi \in \mathscr{F}_{\infty}, \Xi_{t} \in \mathscr{F}_{t}$ for each $t>0$ and an exceptional set $N$ such that $\left(1^{\prime}\right),(2),\left(3^{\prime}\right),(4)$, and $\left(5^{\prime}\right)$ hold:

$\left(1^{\prime}\right) \mathbf{P}_{x}(\Xi)=1$ for all $x \in E \backslash N, \Xi \subset \Xi_{t}$ for all $t>0, \Xi=\bigcap_{t>0} \Xi_{t}$, and $\Xi_{t} \cap\{t<\zeta\} \subset \Xi_{s} \cap\{s<\zeta\}$ for $s<t$; (3') for each $\omega \in \Xi_{t} \cap\{t<\zeta\}$, the same conclusion as in (3) holds; and

$\left(5^{\prime}\right)$ for any $t>0$ and pre-t-equivalent paths $\omega, \omega^{\prime} \in \Omega \cap\{t<\zeta\}$, the same conclusion as in (5) holds.

The notion of rcll-PrAF on $\llbracket 0, \zeta \llbracket$ is similarly defined, and all notions can be formulated for $\operatorname{PrAF}^{*}$ (on $\llbracket 0, \zeta \llbracket$ ) by replacing $\Xi \in \mathscr{F}_{\infty}, \Xi_{t} \in \mathscr{F}_{t}$ with $\Xi \in \mathscr{F}_{\infty}^{*}, \Xi_{t} \in \mathscr{F}_{t}^{*}$.

Definition 4.5 (progressively multiplicative functional). An $\left(\mathscr{F}_{t}\right)$ adapted (resp., $\left(\mathscr{F}_{t}^{*}\right)$-adapted) process $M=\left(M_{t}\right)_{t \geq 0}$ with values in $[0, \infty]$ is said to be a progressively multiplicative functional (PrMF) (resp., relaxed progressively multiplicative functional $\left.\left(\operatorname{Pr} M F^{*}\right)\right)$ if $A$ is $\left(\mathscr{F}_{t}\right)$-adapted (resp., $\left(\mathscr{F}_{t}^{*}\right)$-adapted) and there exist defining sets $\Xi \in \mathscr{F}_{\infty}, \Xi_{t} \in \mathscr{F}_{t}$ (resp., $\Xi \in$ $\left.\mathscr{F}_{\infty}^{*}, \Xi_{t} \in \mathscr{F}_{t}^{*}\right)$ for each $t>0$ and an exceptional set $N$ satisfying conditions (1) and (2) in Definition 4.4 and

(3) for all $\omega \in \Xi_{t}, M .(\omega)$ is defined on $[0, t[$, and it is right-continuous on $[0, t \wedge \zeta(\omega)[$ and has a left limit on $] 0, t] \cap] 0, \zeta(\omega)\left[\right.$ such that $M_{0}(\omega)=1$, and $M_{p+q}(\omega)=M_{p}(\omega) M_{q}\left(\theta_{p} \omega\right)$ for all $p, q \geq 0$ with $p+q<t$;

(4) for all $t \geq 0, M_{t}\left(\omega_{\partial}\right)=1$; and

(5) for any $t>0$ and pre-t-equivalent paths $\omega, \omega^{\prime} \in \Omega, \omega \in \Xi_{t}$ implies that $\omega^{\prime} \in \Xi_{t}, M_{s}(\omega)=M_{s}\left(\omega^{\prime}\right)$ for any $s \in[0, t[$.

Furthermore, $M$ is called an rcll-PrMF if, for each $t>0$ and $\omega \in \Xi_{t}, s \mapsto$ $M_{s}(\omega)$ is right-continuous on $[0, t[$ and has left limits on $] 0, t]$.

The notion of $\operatorname{PrMF}$ on $\llbracket 0, \zeta \llbracket$ is similarly defined, and all notions can be formulated for $\operatorname{PrMF}^{*}$ (on $\llbracket 0, \zeta \llbracket$ ) by replacing $\Xi \in \mathscr{F}_{\infty}, \Xi_{t} \in \mathscr{F}_{t}$ with $\Xi \in \mathscr{F}_{\infty}^{*}, \Xi_{t} \in \mathscr{F}_{t}^{*}$. 
The next notion is stronger than PrAF and suitable to describe our main theorem.

Definition 4.6 (PrAFS). A process $A=\left(A_{t}\right)_{t \geq 0}$ with values in $\overline{\mathbb{R}}:=$ $[-\infty, \infty]$ is said to be a progressively additive functional in the strong sense (PrAFS) (resp., relaxed progressively additive functional in the strong sense $\left.\left(\operatorname{Pr} A F S^{*}\right)\right)$ if $A$ is $\left(\mathscr{F}_{t}\right)$-adapted (resp., $\left(\mathscr{F}_{t}^{*}\right)$-adapted) and there exist defining sets $\Xi \in \mathscr{F}_{\infty}, \Xi_{t} \in \mathscr{F}_{t}$ (resp., $\Xi \in \mathscr{F}_{\infty}^{*}, \Xi_{t} \in \mathscr{F}_{t}^{*}$ ) for each $t>0$ and an exceptional set $N$ satisfying the following conditions:

(1) $\mathbf{P}_{x}(\Xi)=1$ for all $x \in E \backslash N$, for every $t>s>0 \Xi \subset \Xi_{t} \subset \Xi_{s}$, and $\Xi=$ $\bigcap_{t>0} \Xi_{t}$

(2) $\theta_{s} \Xi \subset \Xi$ for all $s \geq 0$ and $\theta_{s}\left(\Xi_{t}\right) \subset \Xi_{t-s}$ for all $\left.s \in\right] 0, t[$; in particular, $\omega_{\partial} \in \Xi \subset \Xi_{t}$ and $\mathbf{P}_{\partial}(\Xi)=\mathbf{P}_{\partial}\left(\Xi_{t}\right)=1$ under $(1)$ :

(3) for all $\omega \in \Xi_{t}, A$.( $\left.\omega\right)$ is defined on $[0, t]$, and it is right-continuous on $[0, t] \cap\left[0, \zeta(\omega)\left[\right.\right.$ and has a left limit $A_{s-}(\omega)$ on $\left.\left.] 0, t\right] \cap\right] 0, \zeta(\omega)[$ such that $A_{0}(\omega)=0,\left|A_{s}(\omega)\right|<\infty$ for $s \in[0, t] \cap\left[0, \zeta(\omega)\left[\right.\right.$, and $A_{p+q}(\omega)=A_{p}(\omega)+$ $A_{q}\left(\theta_{p} \omega\right)$ for all $p, q \geq 0$ with $p+q \leq t ;$

(4) for all $t \geq 0, A_{t}\left(\omega_{\partial}\right)=0$; and

(5) for any $t>0$ and $t$-equivalent paths $\omega, \omega^{\prime} \in \Omega, \omega \in \Xi_{t}$ implies that $\omega^{\prime} \in$ $\Xi_{t}, A_{s}(\omega)=A_{s}\left(\omega^{\prime}\right)$ for any $s \in[0, t]$.

Further, $A$ is called an rcll-PrAFS if, for each $t>0$ and $\omega \in \Xi_{t}, s \mapsto A_{s}(\omega)$ is right-continuous on $[0, t]$ and a has left-hand limit on $] 0, t]$, and a PrAFS is said to be finite (resp., continuous) if $\left|A_{s}(\omega)\right|<\infty$, for all $s \in[0, t]$ (resp., continuous on $[0, t])$ for every $\omega \in \Xi_{t}$.

An $\overline{\mathbb{R}}$-valued process $A$ is called a $\operatorname{Pr} A F S$ on $\llbracket 0, \zeta \llbracket$ if $A$ is $\left(\mathscr{F}_{t}\right)$-adapted and there exist $\Xi \in \mathscr{F}_{\infty}, \Xi_{t} \in \mathscr{F}_{t}$ for each $t>0$ and an exceptional set $N$ such that $\left(1^{\prime}\right),(2),\left(3^{\prime \prime}\right),(4)$, and $\left(5^{\prime \prime}\right)$ hold:

$\left(3^{\prime \prime}\right)$ : for each $\omega \in \Xi_{t} \cap\{t<\zeta\}$, the same conclusion as in (3) holds;

$\left(5^{\prime \prime}\right)$ : for any $t>0$ and $t$-equivalent paths $\omega, \omega^{\prime} \in \Omega \cap\{t<\zeta\}$, the same conclusion as in (5) holds.

The notion of rcll-PrAFS on $\llbracket 0, \zeta \llbracket$ is similarly defined, and all notions can be formulated for PrAFS* (on $\llbracket 0, \zeta \llbracket$ ) by replacing $\Xi \in \mathscr{F}_{\infty}, \Xi_{t} \in \mathscr{F}_{t}$ with $\Xi \in \mathscr{F}_{\infty}^{*}, \Xi_{t} \in \mathscr{F}_{t}^{*}$.

Definition 4.7 (PrMFS). An $\left(\mathscr{F}_{t}\right)$-adapted (resp., $\left(\mathscr{F}_{t}^{*}\right)$-adapted) process $M=\left(M_{t}\right)_{t \geq 0}$ with values in $[0, \infty]$ is said to be a progressively multiplicative functional in the strong sense (PrMFS) (resp., relaxed progressively multiplicative functional in the strong sense $\left.\left(\operatorname{Pr} M F S^{*}\right)\right)$ if $M$ is $\left(\mathscr{F}_{t}\right)$-adapted (resp., $\left(\mathscr{F}_{t}^{*}\right)$-adapted) and there exist defining sets $\Xi \in \mathscr{F}_{\infty}$, 
$\Xi_{t} \in \mathscr{F}_{t}$ (resp., $\Xi \in \mathscr{F}_{\infty}^{*}, \Xi_{t} \in \mathscr{F}_{t}^{*}$ ) for each $t>0$ and an exceptional set $N$ satisfying conditions (1), (2), (3), and (4) in Definition 4.5 and

(5) for any $t>0$ and $t$-equivalent paths $\omega, \omega^{\prime} \in \Omega, \omega \in \Xi_{t}$ implies that $\omega^{\prime} \in$ $\Xi_{t}, M_{s}(\omega)=M_{s}\left(\omega^{\prime}\right)$ for any $s \in[0, t]$.

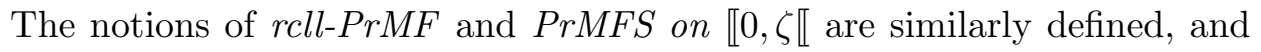
all notions can be formulated for PrMFS* (on $\llbracket 0, \zeta \llbracket$ ) by replacing $\Xi \in \mathscr{F}_{\infty}$, $\Xi_{t} \in \mathscr{F}_{t}$ with $\Xi \in \mathscr{F}_{\infty}^{*}, \Xi_{t} \in \mathscr{F}_{t}^{*}$.

REMARK 4.1.

(1) Our notions of PrAF and PrAFS are different from what is found in Walsh [22]. In [22], the notion of only positive PrAF is treated, and $\left(\mathscr{G}_{t}\right)-$ adaptedness for the positive $\operatorname{PrAF}$ is required, which is very restrictive for our purpose. Here, $\mathscr{G}_{t}=\bigcap_{\mu \in \mathcal{P}(E)} \mathscr{G}_{t}^{\mu}$, and $\mathscr{G}_{t}^{\mu}$ is the $\mathbb{P}_{\mu}$-completion of $\mathscr{F}_{t}^{0}$ smaller than $\mathscr{F}_{t}^{\mu}$. Moreover, the definition of the time reversal operator in [22] is slightly different from ours.

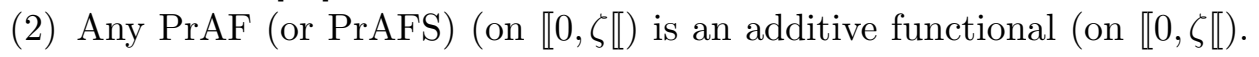
Note that any PrAFS (on $\llbracket 0, \zeta \llbracket$ ) cannot be regarded as a PrAF (on $\llbracket 0, \zeta \llbracket)$ with the same defining sets. But every PrAFS $A$ with defining sets $\Xi_{t}, \Xi$ can be regarded as a PrAF with larger defining sets $\Xi_{t}^{-}:=$ $\left\{\omega \in \bigcap_{s \in] 0, t[} \Xi_{s} \mid A_{t-}(\omega)\right.$ exists in $\left.\mathbb{R}\right\}, \Xi^{-}:=\bigcap_{t>0} \Xi_{t}^{-}$.

(3) Every PCAF $A$ can be regarded as a continuous PrAFS (hence PrAF) (see Lemma 6.1 below or [4, Lemma 2.14 with Remark 2.13]). But it may not be a positive PrAF treated in [22] for the strict $\left(\mathscr{G}_{t}\right)$-adaptedness.

(4) The MAF $M^{u}$ and the CAF $N^{u}$ of 0-energy appearing in the Fukushima decomposition (2.6) can be regarded as finite rcll-PrAFSs in view of [8, proof of Theorem 5.2.2] or Lemma 6.2 below. Hence, an MAF of stochastic integral type $\int_{0}^{t} g\left(X_{s-}\right) \mathrm{d} M_{s}^{u}\left(g, u \in \mathcal{F}\right.$ with $\left.g \in L^{2}\left(E ; \mu_{\langle u\rangle}\right)\right)$ can be regarded as a finite rcll-PrAFS in view of [10, Proposition 4.44]. Consequently, any $M \in \stackrel{\circ}{\mathcal{M}}$ also can be regarded as an rcll-PrAFS in view of the assertion of [8, Lemma 5.6.3] and Lemma 4.4 below. Recall the orthogonal decomposition

$$
M=M^{c}+M^{d}
$$

in the Hilbert space $(\stackrel{\circ}{\mathcal{M}}, \mathbf{e})$, where $M^{c} \in \stackrel{\circ}{\mathcal{M}}^{c}$ (resp., $\left.M^{d} \in \stackrel{\circ}{\mathcal{M}}^{d}\right) . M^{c}$ is the unique element as the minimizer of

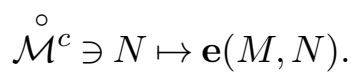


Hence, both $M^{c}$ and $M^{d}$ can be regarded as rcll-PrAFSs in view of the assertion of [8, Lemma 5.6.3] and Lemma 4.4 below.

(5) Every $M \in \mathcal{M}_{\mathrm{loc}}^{\llbracket 0, \zeta \llbracket}$ can be regarded as an rcll-PrAFS on $\llbracket 0, \zeta \llbracket$ from Theorem 5.1 below.

We recall the time reversal operator $r_{t}$ on the rcll path space $\Omega$. It is shown in [4, Lemma 2.10] that for $A \in \mathscr{F}_{t}^{\mathfrak{m}}$ we have $r_{t}^{-1}(A) \in \mathscr{F}_{t}^{\mathfrak{m}}$ and $(2.3)$ holds, where $\mathscr{F}_{t}^{\mathfrak{m}}$ is the $\mathbf{P}_{\mathfrak{m}^{-}}$-completion of $\mathscr{F}_{t}^{0}$ in $\mathscr{F}_{\infty}^{\mathfrak{m}}$.

Definition 4.8 (reversible defining set). Let $A$ be a PrAF (or PrAFS) on $\llbracket 0, \zeta \llbracket$. For each $t>0$, the defining set $\Xi_{t}$ for $A$ is said to be $r_{t}$-reversible or simply reversible if $\omega \in \Xi_{t}$ with $t<\zeta(\omega)$ implies that $r_{t} \omega \in \Xi_{t}$. It is easy to see that the reversibility of $\Xi_{t}$ is equivalent to $\Xi_{t} \subset r_{t}^{-1}\left(\Xi_{t}\right)$, because of $r_{t}^{-1}\left(\Xi_{t}^{c}\right) \cap\{t \geq \zeta\}=\emptyset$. A PrAF (or PrAFS) on $\llbracket 0, \zeta \llbracket$, or its defining sets $\left(\Xi_{t}\right)_{t>0}$, is called time-reversible, or simply reversible, if for every $t>0, \Xi_{t}$ is reversible. The notion of reversibility for PrMF (or PrMFS) on $\llbracket 0, \zeta \llbracket$ is similarly defined.

REMARK 4.2. For an $\omega \in \Omega$ with $t<\zeta(\omega), r_{t}\left(r_{t} \omega\right) \neq \omega$ even if on $[0, t]$. Indeed, $t<\zeta(\omega)$ implies that $t<\zeta\left(r_{t} \omega\right)$ and $r_{t}\left(r_{t} \omega\right)(s)=\omega(s)$ if $s \in[0, t[$ and that $r_{t}\left(r_{t} \omega\right)(s)=\omega(t-)$ if $s \geq t$. Then the reversibility of the defining set $\Xi_{t}$ of a $\operatorname{PrAF}$ on $\llbracket 0, \zeta \llbracket$ is equivalent to that under $t<\zeta(\omega), \omega \in \Xi_{t}$ if and only if $r_{t} \omega \in \Xi_{t}$; that is, if $\Xi_{t} \cap\{t<\zeta\}=r_{t}^{-1}\left(\Xi_{t}\right) \cap\{t<\zeta\}$. If $\Omega$ consists of continuous paths up to the lifetime, then the reversibility of the defining set $\Xi_{t}$ of a PrAFS on $\llbracket 0, \zeta \llbracket$ is equivalent to that under $t<\zeta(\omega), \omega \in \Xi_{t}$ if and only if $r_{t} \omega \in \Xi_{t}$.

LEMma 4.2. Let $\left(\Xi_{t}\right)_{t>0}$ be the family of reversible defining sets of a $\operatorname{PrAFS}$ on $\llbracket 0, \zeta \llbracket$. Then $r_{t}^{-1}\left(\Xi_{t}\right) \in \mathscr{F}_{t}^{*}$ for each $t>0$. If $\mathbf{P}_{x}\left(\Xi_{t}\right)=1$ for all $x \in E$, we have $r_{t}^{-1}\left(\Xi_{t}\right) \in \mathscr{F}_{t}$ for each $t>0$. Moreover, if $\left(\Xi_{t}\right)_{t>0}$ is the defining set of a $\operatorname{Pr} A F$ on $\llbracket 0, \zeta \llbracket$, or if $\Omega$ consists of continuous paths up to the lifetime, then $r_{t}^{-1}\left(\Xi_{t}\right) \in \mathscr{F}_{t}$ for each $t>0$.

Proof. By the definition of $\Xi_{t}$, we have $r_{t}^{-1}\left(\Xi_{t}^{c}\right) \cap\{t<\zeta\} \subset \Xi_{t}^{c} \cap\{t<$ $\zeta\}$; hence, $r_{t}^{-1}\left(\Xi_{t}^{c}\right) \subset \Xi_{t}^{c}$, because $r_{t}^{-1}\left(\Xi_{t}^{c}\right) \cap\{t \geq \zeta\}=\emptyset$. This implies that $r_{t}^{-1}\left(\Xi_{t}^{c}\right) \in \mathscr{F}_{t}^{\nu}$ holds for any $\nu \in S_{00}$ and that it holds for any $\nu \in \mathcal{P}(E)$ provided that $\mathbf{P}_{x}\left(\Xi_{t}\right)=1$ for all $x \in E$. Next, suppose that $\left(\Xi_{t}\right)_{t>0}$ is the defining set of a PrAF or that $\Omega$ consists of continuous paths up to the lifetime. By Remark 4.2, $r_{t}^{-1}\left(\Xi_{t}\right) \cap\{t<\zeta\}=\Xi_{t} \cap\{t<\zeta\}$. Hence, $r_{t}^{-1}\left(\Xi_{t}\right)=$ $\left(\Xi_{t} \cap\{t<\zeta\}\right) \cup\{t \geq \zeta\} \in \mathscr{F}_{t}$. 
Hereafter, we will use the convention that $X_{0-}(\omega):=X_{0}(\omega)$. For notational simplicity, we use the following convention for additive functionals: for an additive functional $A$ and each path $\omega$, we write $A_{s}^{t}(\omega):=A_{t}(\omega)-A_{s}(\omega)$ for $0 \leq s \leq t$, and we write $A_{s-}^{t-}(\omega):=A_{t-}(\omega)-A_{s-}(\omega)$ for $0<s \leq t$.

The following lemma is needed for the present article and can be proved in the same way of the proof of [4, Theorem 2.18]. Its proof is complicated by measurability issues, but the idea behind it is fairly transparent.

Lemma 4.3 (dual PrAF). Let $A$ be an rcll-PrAF on $\llbracket 0, \zeta \llbracket$ with defining sets $\Xi^{-} \in \mathscr{F}_{\infty}, \Xi_{t}^{-} \in \mathscr{F}_{t}$ obtained from a reversible PrAFS A with reversible defining sets $\Xi_{t}, \Xi$; that $i s, \Xi_{t}^{-}:=\left\{\omega \in \bigcap_{s \in] 0, t[} \Xi_{s} \mid A_{t-}(\omega)\right.$ exists in $\left.\mathbb{R}\right\}$ and $\Xi^{-}:=\bigcap_{t>0} \Xi_{t}^{-}$. Suppose that there is a Borel function $\varphi$ on $E \times E$ with $\varphi(x, x)=0$ for $x \in E$ such that $\varphi\left(X_{s-}(\omega), X_{s}(\omega)\right)=A_{s}(\omega)-A_{s-}(\omega)$, for all $s \in] 0, t[\cap] 0, \zeta\left[\right.$ and all $\omega \in \Xi_{t}^{-}$. For $\omega \in r_{t}^{-1}\left(\Xi_{t}^{-}\right)$, we set

$$
\begin{cases}\widehat{A}_{t}(\omega):=A_{t}\left(r_{t}(\omega)\right)+\varphi\left(X_{t}(\omega), X_{t-}(\omega)\right) & \text { for } t \in[0, \zeta(\omega)[, \\ \widehat{A}_{t}(\omega):=0 & \text { for } t \in[\zeta(\omega), \infty[.\end{cases}
$$

Suppose that $A_{t} \circ r_{t}$ is $\mathscr{F}_{t}^{*}$-measurable for each $t>0$. Then $\widehat{A}$ is a finite rcll-PrAF* on $\llbracket 0, \zeta \llbracket$ with defining sets $\widehat{\Xi}_{t}:=r_{t}^{-1}\left(\Xi_{t}^{-}\right)$and $\widehat{\Xi}:=\bigcap_{t>0} \widehat{\Xi}_{t}$ such that

$$
\widehat{A}_{t}=A_{t-} \circ r_{t}+\varphi\left(X_{t}, X_{t-}\right) \quad \text { and } \quad \widehat{A}_{t}-\widehat{A}_{t-}=\varphi\left(X_{t}, X_{t-}\right)
$$

for all $t \in] 0, \zeta\left[, \mathbf{P}_{x}\right.$-a.s. for q.e. $x \in E$.

\section{REMARK 4.3.}

(1) The reversibility of the PrAFS $A$ in Lemma 4.3 is necessary to obtain the $\operatorname{PrAF}^{*} \widehat{A}$. This is one of the key points of our results.

(2) The assumption on the $\mathscr{F}_{t}^{*}$-measurability of $A_{t} \circ r_{t}$ in Lemma 4.3 is satisfied provided that $A$ is $\left(\mathscr{F}_{t}^{0}\right)$-adapted by [4, Lemma 2.10]. If there exist a set $\left\{A^{i} \mid i=1, \ldots, m\right\}$ of finite rcll reversible PrAFSs with the common reversible defining sets $\left(\Xi_{t}\right)_{t>0}$ and a Borel function $f: \mathbb{R}^{\ell} \rightarrow$ $\mathbb{R}$ such that $A_{s}\left(r_{t} \omega\right)=f\left(\left(A^{1}\right)_{(t-s)_{-}}^{t-}(\omega), \ldots,\left(A^{\ell}\right)_{(t-s)_{-}}^{t-}(\omega)\right)$ for $\omega \in \Xi_{t} \cap$ $\{t<\zeta\}$ and $s \in[0, t]$, then $A_{t} \circ r_{t}$ is $\mathscr{F}_{t}^{*}$-measurable. Indeed, for $C \in$ $\mathscr{B}(\mathbb{R})$, we see that $r_{t}^{-1}\left(A_{t}^{-1}(C)\right) \cap \Xi_{t} \cap\{t<\zeta\} \in \mathscr{F}_{t}$ from the expression $A_{t}\left(r_{t} \omega\right)=f\left(A_{t-}^{1}(\omega), \ldots, A_{t-}^{\ell}(\omega)\right)$ for $\omega \in \Xi_{t} \cap\{t<\zeta\}$, and

$$
r_{t}^{-1}\left(A_{t}^{-1}(C)\right) \cap \Xi_{t} \cap\{t \geq \zeta\}= \begin{cases}\emptyset & \text { if } 0 \notin C, \\ \Omega & \text { if } 0 \in C .\end{cases}
$$


Since any subset of $\Xi_{t}^{c}$ belongs to $\mathscr{F}_{t}^{*}$, we have the desired $\mathscr{F}_{t}^{*}$ measurability.

Proof of Lemma 4.3. The first statement follows from Remark 4.1(2). The proof of the middle part is quite similar to [4, proof of Theorem 2.18], which is complicated by measurability issues, so we omit it. We should check that $\widehat{\Xi}_{t} \in \mathscr{F}_{t}^{*}$ and $\mathbf{P}_{x}\left(\widehat{\Xi}_{t}\right)=1, x \in E \backslash N$ for some exceptional set $N$. These follow from the reversibility of $\Xi_{t}$, because of $\Xi_{t} \subset r_{t}^{-1}\left(\Xi_{t}\right) \subset \widehat{\Xi}_{t}$ and because any subset of $\Xi_{t}^{c}$ is $\mathscr{F}_{t}^{*}$-measurable. Therefore, the $\mathscr{F}_{t}^{*}$-measurability of $\widehat{A}_{t}$ completes the proof.

LEMMA 4.4. Let $\left\{\left(A^{n, i}\right)_{n=1}^{\infty} \mid i=1, \ldots, \ell\right\}$ be a finite set of sequences of finite rcll-PrAFSs with common defining sets $\Xi^{n} \in \mathscr{F}_{\infty}, \Xi_{t}^{n} \in \mathscr{F}_{t}$. For each $t>0$, set

$$
\begin{aligned}
\Xi_{t}:= & \left\{\omega \in \bigcap_{n \in \mathbb{N}} \Xi_{t}^{n} \mid \text { for each } i=1,2, \ldots, \ell,\right. \\
& \left.A^{n, i}(\omega) \text { converges uniformly on }[0, t]\right\} \\
\in & \mathscr{F}_{t}
\end{aligned}
$$

and

$$
\begin{aligned}
\Xi:= & \left\{\omega \in \bigcap_{n \in \mathbb{N}} \Xi^{n} \mid \text { for each } i=1,2, \ldots, \ell,\right. \\
& A^{n, i}(\omega) \text { converges uniformly on }[0, t] \text { for every } t \in[0, \infty[\} \\
\in & \mathscr{F}_{\infty} .
\end{aligned}
$$

Suppose that there exists an exceptional set $N$ such that $\mathbf{P}_{x}(\Xi)=1$ for $x \in E \backslash N$. If we define $A_{t}^{i}:=\underline{\lim }_{n \rightarrow \infty} A_{t}^{n, i}$ on $\Omega$, then all $A^{i}$ are finite rcllPrAFSs (resp., PrAFs) with the defining sets $\Xi, \Xi_{t}$ (resp., $\Xi^{-}, \Xi_{t}^{-}$), where $\Xi_{t}^{-}:=\left\{\omega \in \bigcap_{s \in] 0, t[} \Xi_{s} \mid\right.$ all $A_{t-}^{i}(\omega)$ exist in $\left.\mathbb{R}\right\}$ and $\Xi^{-}:=\bigcap_{t>0} \Xi_{t}^{-}$. If, further, all $A^{n, i}$ are finite continuous PrAFSs, then all $A^{i}$ constructed above are finite continuous. Moreover, suppose that, for each $n \in \mathbb{N}, \Xi_{t}^{n}$ is reversible and that there exists a continuous function $f=\left(f_{1}, f_{2}, \ldots, f_{\ell}\right): \mathbb{R}^{\ell} \rightarrow \mathbb{R}^{\ell}$ such that, for each $i=1, \ldots, \ell$ and $\omega \in \Xi_{t}^{n}$ with $t<\zeta(\omega), A_{s}^{n, i}\left(r_{t} \omega\right)=$ $f_{i}\left(\left(A^{n, 1}\right)_{(t-s)-}^{t-}(\omega), \ldots,\left(A^{n, \ell}\right)_{(t-s)-}^{t-}(\omega)\right)$ for $s \in[0, t]$. Then $\Xi_{t}$ is reversible. 
Proof. The proof of the first part is quite similar to the proof of $[4$, Lemma 2.14], so all $A^{i}(i=1, \ldots, \ell)$ are PrAFSs with defining sets $\Xi, \Xi_{t}$ as claimed. As noted in Remark 4.1(2), those are also PrAFs with defining sets $\Xi^{-}, \Xi_{t}^{-}$. Now we prove the second statement. Suppose that each $\Xi_{t}^{n}$ is reversible for each $n \in \mathbb{N}$. The reversibility of $\Xi_{t}$ is clear from the expressions for $A_{s}^{n, i} \circ r_{t}$ on $\Xi_{t} \cap\{t<\zeta\}$ for $s \in[0, t]$.

\section{$\S 5$. Main theorems}

In this section, we give our main theorem. Recall that, for $M \in\left(\mathcal{M}_{\mathrm{loc}}^{\llbracket 0, \zeta \llbracket}\right)_{*}$ with its jump function $\varphi \in \mathcal{J}_{*}$, there exists $K \in\left(\mathcal{M}_{\text {loc }}^{d}\right)_{*}$ (resp., $K^{\ell} \in$ $\left.\left(\mathcal{M}_{\text {loc }}^{d}\right)_{*}\right)$ such that its jump function is given by $-\mathbf{1}_{E \times E}(\varphi+\bar{\varphi}) \in \mathcal{J}_{*}$ (resp., $\left.-\mathbf{1}_{E \times E}\left(\varphi_{\ell}+\overline{\varphi_{\ell}}\right) \in \mathcal{J}_{*}\right)$. We write $(f * \Gamma(M))_{t}$ instead of $\int_{0}^{t} f\left(X_{s}\right) \mathrm{d} \Gamma(M)_{s}$ for $M \in\left(\mathcal{M}_{\text {loc }}\right)_{*}$ and $f \in \dot{\mathcal{F}}_{\text {loc }}$.

Theorem 5.1. Let $Y \in \mathcal{M}_{\mathrm{loc}}^{\llbracket 0, \zeta \llbracket . ~ T h e n ~} Y$ can be extended to be an rcll reversible $\operatorname{Pr} A F S$ on $\llbracket 0, \zeta \llbracket$ with reversible defining sets $\left(\Xi_{t}\right)_{t>0}$. Moreover, $Y$ can be extended to be an rcll-PrAF on $\llbracket 0, \zeta \llbracket$ with some defining sets $\Xi_{t}^{-}$, $\Xi^{-}$containing $\Xi_{t}, \Xi$, respectively, and $\widehat{Y}$ defined as in (4.1) is a $\operatorname{Pr} A F^{*}$ on $\llbracket 0, \zeta \llbracket$ with defining sets $\widehat{\Xi}_{t}:=r_{t}^{-1}\left(\Xi_{t}^{-}\right), \widehat{\Xi}:=\bigcap_{t>0} \widehat{\Xi}_{t}$.

Theorem 5.2. Take $M \in\left(\mathcal{M}_{\mathrm{loc}}^{\llbracket 0, \zeta \llbracket}\right)_{*}$, and take $f \in \dot{\mathcal{F}}_{\text {loc }}$. Then additive functionals $f * M, f * K,[f(X), M+K], f * \Gamma(M)$, and $f * \Gamma(K)$ can be extended to be rcll reversible PrAFSs on $\llbracket 0, \zeta \llbracket$ with common reversible defining sets $\left(\Xi_{t}\right)_{t>0}$ such that, for $\omega \in \Xi_{T}, T<\zeta(\omega)$, and $t \in[0, T]$, $(f * \Gamma(K))_{t}(\omega)=0$ and

$$
\begin{aligned}
(f * M)_{t}\left(r_{T} \omega\right)= & -(f *(M+K))_{(T-t)-}^{T-}(\omega) \\
& -[f(X), M+K]_{(T-t)-}^{T-}(\omega)-2(f * \Gamma(M))_{T-t}^{T}(\omega) .
\end{aligned}
$$

In particular, there exist reversible defining sets $\left(\Xi_{t}\right)_{t>0}$ of $f * K$ such that for $\omega \in \Xi_{T}, T<\zeta(\omega)$, and $t \in[0, T]$,

$$
(f * K)_{t}\left(r_{T} \omega\right)=(f * K)_{(T-t)-}^{T-}(\omega)+[f(X), K]_{(T-t)-}^{T-}(\omega) .
$$

Moreover, the same assertion as in Theorem 5.1 for PrAFSs including $f * K$, $[f(X), M+K], f * \Gamma(M)$, and $f * \Gamma(K)$ holds. 
REMARK 5.1. The conclusions of Theorems 5.1 and 5.2 also include the assertion that, for each $t>0, \Xi_{t} \subset \widehat{\Xi}_{t}$ holds for the defining set $\Xi_{t}$ of the given PrAFS on $\llbracket 0, \zeta \llbracket$ and the defining set $\widehat{\Xi}_{t}$ of the constructed $\operatorname{PrAF}^{*}$ on $\llbracket 0, \zeta \llbracket$.

One of the consequences of Theorem 5.2 is the following refinement of the Lyons-Zheng decomposition.

Theorem 5.3 (Lyons-Zheng decomposition for the Fisk-Stratonovich integral). Take $M \in\left(\mathcal{M}_{\text {loc }}^{\llbracket 0, \zeta \llbracket}\right)_{*}$, and take $f \in \dot{\mathcal{F}}_{\text {loc }}$. Set $\check{M}:=M+\frac{1}{2} K$, and set $\bar{A}:=A+(1 / 2) K=\check{M}+\Gamma(\check{M})$. Then for q.e. $x \in E$, the following holds $\mathbf{P}_{x}$-a.s. on $\{T<\zeta\}$ : for all $t \in[0, T]$

$$
\begin{aligned}
\int_{0}^{t} f\left(X_{s}\right) \circ \mathrm{d} \bar{A}_{s}= & \frac{1}{2} \int_{0}^{t} f\left(X_{s-}\right) \mathrm{d} \check{M}_{s} \\
& -\frac{1}{2} \int_{(T-t)+}^{T-} f\left(X_{T-s}\right) \mathrm{d}\left(\check{M}_{s} \circ r_{T}\right), \\
\bar{A}_{t}= & \frac{1}{2} \check{M}_{t}-\frac{1}{2}\left(\check{M}_{T-} \circ r_{T}-\check{M}_{(T-t)-} \circ r_{T}\right) .
\end{aligned}
$$

Here $\int_{0}^{t} f\left(X_{s}\right) \circ \mathrm{d} \bar{A}_{s}$ is the Stratonovich-type integral defined for $t \in[0, \zeta[$ in [13, Definition 4.1] (see also [14]).

Proof. Let $\left(\Xi_{t}\right)_{t>0}$ be the common reversible defining sets that appeared in Theorem 5.2. By (5.1) and (5.2), we see that, for $\omega \in \Xi_{T}, T<\zeta(\omega)$, $t \in[0, T]$,

right-hand side of (5.3)

$$
\begin{aligned}
&= \frac{1}{2}(f * \check{M})_{t}(\omega)-\frac{1}{2}\left((f * \check{M})_{T-}\left(r_{T} \omega\right)-(f * \check{M})_{(T-t)-}\left(r_{T} \omega\right)\right) \\
&= \frac{1}{2}(f * M)_{t}(\omega)-\frac{1}{2}\left((f * M)_{T-}\left(r_{T} \omega\right)-(f * M)_{(T-t)-}\left(r_{T} \omega\right)\right) \\
&+\frac{1}{4}(f * K)_{t}(\omega)-\frac{1}{4}\left((f * K)_{T-}\left(r_{T} \omega\right)-(f * K)_{(T-t)-}\left(r_{T} \omega\right)\right) \\
& \stackrel{(5.1),(5.2)}{=} \frac{1}{2}(f * M)_{t}(\omega)+\frac{1}{2}\left\{(f *(M+K))_{t}(\omega)+[f(X), M+K]_{t}(\omega)\right. \\
&\left.\quad+2(f * \Gamma(M))_{t}(\omega)\right\} \\
& \quad+\frac{1}{4}(f * K)_{t}(\omega)-\frac{1}{4}\left((f * K)_{t}(\omega)+[f(X), K]_{t}(\omega)\right)
\end{aligned}
$$




$$
\begin{aligned}
= & \left(f *\left(M+\frac{1}{2} K\right)\right)_{t}(\omega)+\frac{1}{2}\left[f(X), M+\frac{1}{2} K\right]_{t}(\omega) \\
& +(f * \Gamma(M))_{t}(\omega) \\
= & \int_{0}^{t} f\left(X_{s}(\omega)\right) \circ \mathrm{d} \bar{A}_{s}(\omega) .
\end{aligned}
$$

The following corollaries are consequences of Theorem 5.3.

COROLlary 5.1 (Lyons-Zheng decomposition for the Fisk-Stratonovich integral by $\left.A:=M+\Gamma(M), M \in\left(\mathcal{M}_{\mathrm{loc}}^{\llbracket 0, \zeta \llbracket}\right)_{\text {as }}\right)$. Take $M \in\left(\mathcal{M}_{\mathrm{loc}}^{\llbracket 0, \zeta \llbracket}\right)_{\text {as }}$, and take $f \in \dot{\mathcal{F}}_{\text {loc }}$. Set $A:=M+\Gamma(M)$. Then for q.e. $x \in E$, the following holds $\mathbf{P}_{x}$-a.s. on $\{T<\zeta\}$ : for all $t \in[0, T]$,

$$
\begin{aligned}
\int_{0}^{t} f\left(X_{s}\right) \circ \mathrm{d} A_{s}= & \frac{1}{2} \int_{0}^{t} f\left(X_{s-}\right) \mathrm{d} M_{s} \\
& -\frac{1}{2} \int_{(T-t)+}^{T-} f\left(X_{T-s}\right) \mathrm{d}\left(M_{s} \circ r_{T}\right), \\
A_{t}= & \frac{1}{2} M_{t}-\frac{1}{2}\left(M_{T-} \circ r_{T}-M_{(T-t)-} \circ r_{T}\right) .
\end{aligned}
$$

Here $\int_{0}^{t} f\left(X_{s}\right) \circ \mathrm{d} A_{s}$ is the Stratonovich-type integral defined for $t \in[0, \zeta[$ in [13, Definition 4.1] (see also [14]).

Corollary 5.2 (Lyons-Zheng decomposition for the Fisk-Stratonovich integral by $\left.A^{u, d}, u \in \dot{\mathcal{F}}_{\text {loc }}^{\dagger}\right)$. Take $f \in \dot{\mathcal{F}}_{\text {loc }}$, and take $u \in \dot{\mathcal{F}}_{\text {loc }}^{\dagger}$. We set $A^{u, d}:=$ $M^{u, d}+\Gamma\left(M^{u, d}\right)$. Here $M^{u, d}$ is an element in $\left(\mathcal{M}_{\mathrm{loc}}^{d, \llbracket 0, \zeta \llbracket}\right)_{\text {as }}$ such that $\Delta M_{t}^{u, d}=$ $\left.u\left(X_{t}\right)-u\left(X_{t-}\right) t \in\right] 0, \zeta\left[\mathbf{P}_{x}\right.$-a.s. for q.e. $x \in E$. Then for q.e. $x \in E$, the following holds $\mathbf{P}_{x}$-a.s. on $\{T<\zeta\}$ : for all $t \in[0, T]$

$$
\begin{aligned}
\int_{0}^{t} f\left(X_{s}\right) \circ \mathrm{d} A_{s}^{u, d}= & \frac{1}{2} \int_{0}^{t} f\left(X_{s-}\right) \mathrm{d} M_{s}^{u, d} \\
& -\frac{1}{2} \int_{(T-t)+}^{T-} f\left(X_{T-s}\right) \mathrm{d}\left(M_{s}^{u, d} \circ r_{T}\right), \\
A_{t}^{u, d}= & \frac{1}{2} M_{t}^{u, d}-\frac{1}{2}\left(M_{T-}^{u, d} \circ r_{T}-M_{(T-t)-}^{u, d} \circ r_{T}\right) .
\end{aligned}
$$

Here $\int_{0}^{t} f\left(X_{s}\right) \circ \mathrm{d} A_{s}^{u, d}$ is the Stratonovich-type integral defined for $t \in[0, \zeta[$ in [13, Definition 4.1] (see also [14]). 
Corollary 5.3 (Lyons-Zheng decomposition for $A^{u}, u \in \dot{\mathcal{F}}_{\text {loc }}^{\dagger}$ ). Take $u \in \dot{\mathcal{F}}_{\text {loc }}^{\dagger}$. For q.e. $x \in E$, the following holds $\mathbf{P}_{x}$-a.s. on $\{T<\zeta\}$, respectively: for all $t \in[0, T]$,

$$
u\left(X_{t}\right)-u\left(X_{0}\right)=\frac{1}{2} M_{t}^{u}-\frac{1}{2}\left(M_{T-}^{u} \circ r_{T}-M_{(T-t)-}^{u} \circ r_{T}\right),
$$

where we regard $X_{0_{-}}=X_{0}$ and $M_{0_{-}}:=0$.

Corollary 5.4 (Lyons-Zheng decomposition for $M \in \mathcal{M}_{\mathrm{loc}}^{c, \llbracket 0, \zeta \llbracket}$ ). Take

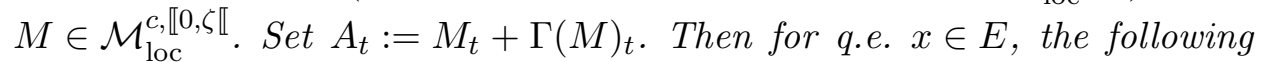
holds $\mathbf{P}_{x}$-a.s. on $\{T<\zeta\}$ : for all $t \in[0, T]$,

$$
A_{t}=\frac{1}{2} M_{t}-\frac{1}{2}\left(M_{T} \circ r_{T}-M_{T-t} \circ r_{T}\right) .
$$

Corollary 5.5 (Lyons-Zheng decomposition for the Fisk-Stratonovich integral by $\left.A^{u, c}\right)$. Take $u \in \dot{\mathcal{F}}_{\text {loc }}, f \in L_{\text {loc }}^{2}\left(\left\{G_{n}\right\} ; \mu_{\left\langle M^{u, c}\right\rangle}\right)$ for some nest $\left\{G_{n}\right\} \in \Theta$, and take $\left\{u_{n}\right\} \subset \mathcal{F}_{b}$ such that $u=u_{n} \mathfrak{m}$-a.e. on $G_{n}$ for each $n \in \mathbb{N}$. Then for q.e. $x \in E$, the following holds $\mathbf{P}_{x}$-a.s. on $\{T<\zeta\}$, respectively: for all $t \in[0, T]$,

$$
\begin{aligned}
\int_{0}^{t} f\left(X_{s}\right) \circ \mathrm{d} A_{s}^{u, c}= & \frac{1}{2} \int_{0}^{t} f\left(X_{s-}\right) \mathrm{d} M_{s}^{u, c} \\
& -\frac{1}{2} \int_{T-t}^{T} f\left(X_{T-s}\right) \mathrm{d}\left(M_{s}^{u, c} \circ r_{T}\right), \\
A_{t}^{u, c}= & \frac{1}{2} M_{t}^{u, c}-\frac{1}{2}\left(M_{T}^{u, c} \circ r_{T}-M_{T-t}^{u, c} \circ r_{T}\right) .
\end{aligned}
$$

Here $\int_{0}^{t} f\left(X_{s}\right) \circ \mathrm{d} A_{s}^{u, c}$ is the Stratonovich-type integral defined for $t \in[0, \zeta[$ in [13, Definition 4.1] (see also [14]).

REMARK 5.2. Corollary 5.5 strengthens Lyons-Zheng decompositions on Fisk-Stratonovich-type integrals by Nakao [18], Lyons and Zheng [16], and Lyons and Zhang [15] formulated under the condition that $J=\kappa=0$. Actually, $[15,(2.1 .6)]$ essentially proves that (5.11) holds for every $t \in[0, T] \mathbf{P}_{\mathfrak{m}^{-}}$ a.e. under the condition that $\mathbf{X}$ is a conservative diffusion process having no inside killing and the associated Dirichlet form admits the square-field operator. 
As another consequence of Theorem 5.2, we can connect two kinds of extensions of Nakao operators and Nakao integrals defined in [4] and [13]. More precisely, as in [4, Definition 3.3], we can define $\Lambda(M)$ for $M \in\left(\mathcal{M}_{\text {loc }}^{\llbracket 0, \zeta \llbracket}\right)_{*}$ having jump function $\varphi \in \mathcal{J}_{*}: \Lambda(M)_{0}:=0$ and

$$
\left.\Lambda(M)_{t}:=-\frac{1}{2}\left(M_{t}+M_{t} \circ r_{t}+\varphi\left(X_{t}, X_{t-}\right)+K_{t}\right), \quad t \in\right] 0, \zeta[
$$

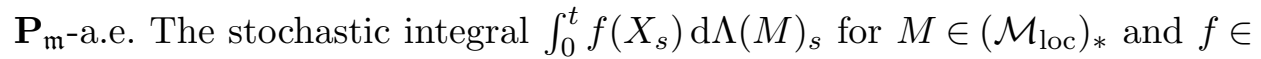
$\dot{\mathcal{F}}_{\text {loc }}^{\dagger}$ is now defined in the following way:

$$
\begin{aligned}
\int_{0}^{t} f\left(X_{s}\right) \mathrm{d} \Lambda(M)_{s}:= & \Lambda(f * M)_{t} \\
& -\frac{1}{2}\left\langle M^{f, c}+M^{f, j}, M^{c}+M^{j}+K\right\rangle_{t} \quad t \in[0, \zeta[
\end{aligned}
$$

$\mathbf{P}_{\mathfrak{m}}$-a.e. Here $M^{f, j}$ is the locally square integrable MAF of purely discontinuous type such that $\left.\Delta M_{t}^{f, j}=\left(f\left(X_{t}\right)-f\left(X_{t-}\right)\right) \mathbf{1}_{\{t<\zeta\}} t \in\right] 0, \infty\left[\mathbf{P}_{x^{-}}\right.$a.s. for q.e. $\in E$.

Theorem 5.4. For $M \in\left(\mathcal{M}_{\mathrm{loc}}^{\llbracket 0, \zeta \llbracket}\right)_{*}, \Lambda(M)_{t} t \in\left[0, \zeta\left[\right.\right.$ can be defined $\mathbf{P}_{x}$-a.s. for q.e. $x \in E$ and

$$
\left.\Gamma(M)_{t}=\Lambda(M)_{t} \quad t \in\right] 0, \zeta\left[\mathbf{P}_{x} \text {-a.s. for q.e. } x \in E .\right.
$$

Moreover, for $f \in \dot{\mathcal{F}}_{\text {loc }}^{\dagger}$ and also for $M \in\left(\mathcal{M}_{\mathrm{loc}}\right)_{*}$, it holds that the stochastic integral $\int_{0}^{t} f\left(X_{s}\right) \mathrm{d} \Lambda(M)_{s} t \in\left[0, \zeta\left[\right.\right.$ can be defined $\mathbf{P}_{x}$-a.s. for q.e. $x \in E$ and

$$
\begin{array}{rl}
\int_{0}^{t} & f\left(X_{s}\right) \mathrm{d} \Gamma(M)_{s} \\
\quad=\int_{0}^{t} f\left(X_{s}\right) \mathrm{d} \Lambda(M)_{s} \quad \text { for all } t \in\left[0, \zeta\left[\mathbf{P}_{x} \text {-a.s. for q.e. } x \in E .\right.\right.
\end{array}
$$

Proof. For $\omega \in \Xi_{T}$ with $T<\zeta(\omega)$ and $t \in[0, T]$, we have $\omega \in \Xi_{t}$; hence, by applying (5.1) to $\omega \in \Xi_{t}$ with $t<\zeta(\omega)$,

$$
\begin{aligned}
(f * \Gamma(M))_{t}(\omega)= & -\frac{1}{2}\left((f *(M+K))_{t-}(\omega)\right. \\
& \left.+\left[M^{f}, M+K\right]_{t-}(\omega)+(f * M)_{t}\left(r_{t} \omega\right)\right)
\end{aligned}
$$

in particular, if $f=\mathbf{1}_{E}$, we then have 


$$
\begin{aligned}
\Gamma(M)_{t}(\omega) & =-\frac{1}{2}\left(M_{t-}(\omega)+M_{t}\left(r_{t} \omega\right)+K_{t-}(\omega)\right) \\
& =-\frac{1}{2}\left(M_{t}(\omega)+M_{t}\left(r_{t} \omega\right)+\varphi\left(X_{t}(\omega), X_{t-}(\omega)\right)+K_{t}(\omega)\right) \\
& =\Lambda(M)_{t}(\omega) .
\end{aligned}
$$

Let $\bar{K}$ be the local MAF of purely discontinuous type such that $\Delta \bar{K}_{t}=$ $-\left(f\left(X_{t-}\right) \varphi\left(X_{t-}, X_{t}\right)+f\left(X_{t}\right) \varphi\left(X_{t}, X_{t-}\right)\right) \mathbf{1}_{\{t<\zeta\}}$ for all $\left.t \in\right] 0, \infty\left[\mathbf{P}_{x^{-}}\right.$a.s. for q.e. $x \in E$ (see [4, Lemma $3.2($ ii) $]$ ); $\bar{K}$ can be also regarded as a PrAFS with reversible defining sets. Indeed, let $\left(\widehat{\Xi}_{t}^{\ell}\right)_{t>0}$ be the time-reversible defining sets for the CAF $\int_{0}^{t} N\left(\mathbf{1}_{E \times E}\left(f \varphi_{\ell}+\overline{f \varphi_{\ell}}\right)\right)\left(X_{s}\right) \mathrm{d} H_{s}$. Then

$$
\begin{aligned}
\Xi_{T}^{\ell}:= & \left\{\omega \in \widehat{\Xi}_{T}^{\ell} \mid \sum_{0<s \leq t}\left(f \varphi_{\ell}+\overline{f \varphi_{\ell}}\right)\left(X_{s-}, X_{s}\right) \mathbf{1}_{\{s<\zeta\}}\right. \\
& \text { absolutely converges on }[0, T]\}
\end{aligned}
$$

is the time-reversible defining set for the purely discontinuous local MAF $\bar{K}^{\ell} \in\left(\mathcal{M}_{\text {loc }}^{d}\right)_{*}$ whose jump function is given by $-\mathbf{1}_{E \times E}\left(f \varphi_{\ell}+\overline{f \varphi_{\ell}}\right)$. Since $f \varphi_{\ell}+\overline{f \varphi_{\ell}}=f\left(\varphi_{\ell}+\overline{\varphi_{\ell}}\right)+(\bar{f}-f) \overline{\varphi_{\ell}}$, we can see that $\mathbf{1}_{G_{n}} * \bar{K}^{\ell} \in \stackrel{\circ}{\mathcal{M}}$ and that $\left\{\mathbf{1}_{G_{n}} *\left(\bar{K}^{\ell}-\bar{K}^{1}\right)\right\}_{\ell}$ forms an e-Cauchy sequence in $\stackrel{\circ}{\mathcal{M}}$. Taking a subsequence $\left\{\ell_{k}\right\}$ of $\{\ell\}$, we see that $\bar{K}^{\ell_{k}}$ uniformly converges to $\bar{K}$ on each compact interval $\mathbf{P}_{x}$-a.s. for q.e. $x \in E$. Then one can construct an adequate timereversible common defining set $\Xi_{T}$ for $\bar{K}$ and $f * M$. We define, for $\omega \in \Xi_{T}$ with $T<\zeta(\omega)$ and $t \in[0, T]$,

$$
\Lambda(f * M)_{t}(\omega):=-\frac{1}{2}\left((f * M)_{t-}(\omega)+(f * M)_{t}\left(r_{t} \omega\right)+\bar{K}_{t}(\omega)\right) .
$$

This means that $\Lambda(f * M)$ defined under $\mathbf{P}_{\mathfrak{m}}$ in [4, Definition 3.3] can be redefined $\mathbf{P}_{x}$-a.s. for q.e. $x \in E$. Putting $\bar{M}^{j}:=-\left(M^{j}+K\right)$ and $\overline{f * M^{j}}:=$ $-\left(f * M^{j}+\bar{K}\right)$, we see that $\omega \in \Xi_{T}$ with $T<\zeta(\omega)$ and $t \in[0, T]$

$$
\begin{aligned}
& \int_{0}^{t} f\left(X_{s}(\omega)\right) \mathrm{d} \Lambda(M)_{s}(\omega) \\
& \quad=\Lambda(f * M)_{t}(\omega)-\frac{1}{2}\left\langle M^{f, c}+M^{f, j}, M^{c}-\bar{M}^{j}\right\rangle_{t}(\omega) \\
& =-\frac{1}{2}\left((f * M)_{t-}(\omega)+(f * M)_{t}\left(r_{t} \omega\right)-\left(f * M^{j}\right)_{t}(\omega)\right.
\end{aligned}
$$




$$
\begin{aligned}
& \left.-\left(\overline{f * M^{j}}\right)(\omega)+\left\langle M^{f, c}, M^{c}\right\rangle_{t}(\omega)-\left\langle M^{f, j}, \bar{M}^{j}\right\rangle_{t}(\omega)\right) \\
= & -\frac{1}{2}(f *(M+K))_{t-}(\omega)+\left[M^{f}, M+K\right]_{t-}(\omega)+(f * M)_{t}\left(r_{t} \omega\right) \\
= & (f * \Gamma(M))_{t}(\omega),
\end{aligned}
$$

which implies the desired assertion.

ExAmple 5.1 (d-dimensional Brownian motion). Let $\mathbf{X}=\left(\Omega, X_{t}, \mathbf{P}_{x}\right)$ be the $d$-dimensional Brownian motion. We take a measurable vector field $b$ : $\mathbb{R}^{d} \rightarrow \mathbb{R}^{d}$, and we assume that the measure $|b(x)|^{2} \mathrm{~d} x$ is a smooth measure. We consider the Itô integral $M_{t}:=\int_{0}^{t} b\left(X_{s}\right) \mathrm{d} X_{s}$. Then $M \in \mathcal{M}_{\mathrm{loc}}^{c}=\mathcal{M}_{\mathrm{loc}}^{c}$ and $N:=\Gamma(M) \in \mathcal{N}_{c, \text { loc }}$. Note that if we assume that $b \in C^{1}\left(\mathbb{R}^{d} \rightarrow \mathbb{R}^{d}\right)$, then we have the expression $N_{t}=\int_{0}^{t}(\operatorname{div} b)\left(X_{s}\right) \mathrm{d} s$. By Theorem 5.2, for each $f \in H_{\mathrm{loc}}^{1}\left(\mathbb{R}^{d}\right), M, N, f * M$ and $f * N$ can be regarded as finite continuous PrAFSs with a common time-reversible defining set $\left(\Xi_{t}\right)_{t>0}$ such that, for $T>0, \omega \in \Xi_{T}$ and $t \in[0, T]$,

$$
\begin{aligned}
M_{t}\left(r_{T} \omega\right) & =-M_{T-t}^{T}(\omega)-2 N_{T-t}^{T}(\omega), \quad N_{t}\left(r_{T}(\omega)\right)=N_{T-t}^{T}(\omega), \\
(f * M)_{t}\left(r_{T} \omega\right) & =-(f * M)_{T-t}^{T}(\omega)-2(f * N)_{T-t}^{T}(\omega), \\
(f * N)_{t}\left(r_{T}(\omega)\right) & =(f * N)_{T-t}^{T}(\omega) .
\end{aligned}
$$

That is, these hold for $\mathbf{P}_{x}$-a.s. for q.e. $x \in \mathbb{R}^{d}$ (for all $x \in \mathbb{R}$ provided that $d=1)$.

EXAmple 5.2 (symmetric Lévy process on $\left.\mathbb{R}^{d}\right)$. Let $\mathbf{X}=\left(\Omega, X_{t}, \zeta\right.$, $\left.\mathbf{P}_{x}\right)_{x \in \mathbb{R}^{d}}$ be the symmetric Lévy process. That is, $\mathbf{X}$ is a time-homogeneous additive process determined by a family $\left\{\nu_{t}\right\}$ of probability measures on $\mathbb{R}^{d}$ satisfying $[8,(4.17),(4.18),(4.19)]$. Let $(\mathcal{E}, \mathcal{F})$ be the corresponding Dirichlet form on $L^{2}\left(\mathbb{R}^{d}\right)$. Then $(\mathcal{E}, \mathcal{F})$ is given by

$$
\left\{\begin{array}{l}
\mathcal{E}(u, v)=\int_{\mathbb{R}^{d}} \hat{u}(\xi) \overline{\hat{v}}(\xi) \psi(\xi) \mathrm{d} \xi, \\
\mathcal{F}=\left\{\left.u \in L^{2}\left(\mathbb{R}^{d}\right)\left|\int_{\mathbb{R}^{d}}\right| \hat{u}(\xi)\right|^{2} \psi(\xi) \mathrm{d} \xi<\infty\right\},
\end{array}\right.
$$

where $\hat{u}(\xi):=\left(1 /(2 \pi)^{d / 2}\right) \int_{\mathbb{R}^{d}} e^{i\langle\xi, x\rangle} u(x) \mathrm{d} x$ and where $\psi(x)$ is the function determined by $\mathbf{E}_{0}\left[e^{i\left\langle\xi, X_{t}\right\rangle}\right]=e^{-t \psi(\xi)}$. We assume that $\mathbf{X}$ is purely discontinuous, namely, that $\psi$ has the expression

$$
\psi(\xi)=\int_{\mathbb{R}^{d}}(1-\cos \langle\xi, \eta\rangle) \nu(\mathrm{d} \eta),
$$


where $\nu$ is a symmetric measure on $\mathbb{R}^{d} \backslash\{0\}$ such that $\int_{\mathbb{R}^{d} \backslash\{0\}}\left(|\xi|^{2} \wedge 1\right) \nu(\mathrm{d} \xi)<$ $\infty$, which is called the Lévy measure of $\mathbf{X}$. We see that $C_{0}^{\text {Lip }}\left(\mathbb{R}^{d}\right) \subset H_{1}\left(\mathbb{R}^{d}\right) \subset$ $\mathcal{F}$; hence, $C_{\text {loc }}^{\text {Lip }}\left(\mathbb{R}^{d}\right) \subset H_{\text {loc }}^{1}\left(\mathbb{R}^{d}\right) \subset \mathcal{F}_{\text {loc }}$, because of

$$
1+\psi(\xi) \leq c\left(1+|\xi|^{2}\right) \quad{ }^{\forall} \xi \in \mathbb{R}^{d}
$$

for some constant $c>0$ (see [1, Corollary 7.16]). Here $C_{0}^{\mathrm{Lip}}\left(\mathbb{R}^{d}\right)$ (resp., $\left.C_{\text {loc }}^{\text {Lip }}\left(\mathbb{R}^{d}\right)\right)$ is the family of Lipschitz continuous functions with compact support (resp., locally Lipschitz continuous functions), and $\mathcal{F}_{\text {loc }}$ is the space of functions locally in $\mathcal{F}$ in the ordinary sense (see [8]). Further, $(\mathcal{E}, \mathcal{F})$ is a regular Dirichlet form having $C_{0}^{\infty}\left(\mathbb{R}^{d}\right)$ as its core (see [21]). Define $N(x, A):=\nu(A-x), N(x,\{\partial\})=0$ for $A \in \mathscr{B}\left(\mathbb{R}^{d}\right), x \in \mathbb{R}^{d}$, and $H_{t}=t$. By [19, Theorem 19.2(i)], we have

$$
N(x, A)=\mathbf{E}_{x}\left[\sum_{0<s \leq 1} \mathbf{1}_{A}\left(X_{s}-X_{s-}\right)\right] \quad A \in \mathscr{B}\left(\mathbb{R}^{d}\right) ;
$$

hence, $(N, H)$ becomes a Lévy system of $\mathbf{X}$. Further, we assume that $\nu(\mathrm{d} y)=$ $f(|y|) \mathrm{d} y$, where $f$ is a Borel function satisfying

$$
\int_{c}^{\infty} f(r) r^{d+1} \mathrm{~d} r<\infty \text { for some } c>0 .
$$

Equation (5.14) is not satisfied for $f(r)=\frac{A(d,-\alpha)}{2} r^{-d-\alpha}$, where $\left.\alpha \in\right] 0,2[$, $A(d,-\alpha):=\frac{\alpha 2^{d+\alpha} \Gamma\left(\frac{d+\alpha}{2}\right)}{2^{d+1} \pi^{d / 2} \Gamma\left(1-\frac{\alpha}{2}\right)}$, the case of symmetric $\alpha$-stable processes, but is satisfied for processes with finite range jumps, or processes with jumps exponentially decayed, and so on. More concretely, (5.14) is satisfied for relativistic symmetric $\alpha$-stable processes with mass $m>0$ by setting $f(r)=\frac{A(d,-\alpha)}{2} r^{-d-\alpha} \Psi\left(m^{1 / \alpha} r\right)$, where $\Psi(r):=I(r) / I(0)$ with $I(r):=$ $\int_{0}^{\infty} s^{\frac{d+\alpha}{2}-1} e^{-\frac{s}{4}-\frac{r^{2}}{s}} \mathrm{~d} s$ satisfies $\Psi \asymp e^{-r}\left(1+r^{d+\alpha-1}\right)$ at $r=\infty$. Let $u \in$ $C^{\operatorname{Lip}}\left(\mathbb{R}^{d}\right)$. Then under (5.14),

$$
\sup _{x \in K} \int_{G^{c}}(u(x)-u(y))^{2} f(|x-y|) \mathrm{d} y<\infty
$$

for any compact set $K$ and its relatively compact open neighborhood $G$; hence, $u \in \mathcal{F}_{\text {loc }}^{\dagger}$ (equivalently, $\left.(u(\cdot)-u)^{2} \in \mathcal{J}\right)$. Then there exists $M^{u} \in$ $\left(\mathcal{M}_{\text {loc }}^{\llbracket 0, \zeta \llbracket}\right)_{\text {as }}$ such that $M_{t}^{u}-M_{t-}^{u}=u\left(X_{t}\right)-u\left(X_{t-}\right) t<\zeta \mathbf{P}_{x^{-a}}$.s. for q.e. $x \in \mathbb{R}^{d}$. We set $N^{u}:=\Gamma\left(M^{u}\right) \in \mathcal{N}_{c, \text { loc }}$. By Theorem 5.2, for each $f \in \mathcal{F}_{\text {loc }}$, 
$M^{u}, N^{u}, f * M^{u}$ and $f * N^{u}$ can be regarded as PrAFSs with common reversible defining sets $\left(\Xi_{t}\right)_{t>0}$ such that for $T>0, \omega \in \Xi_{T}$ with $T<\zeta(\omega)$ and $t \in[0, T]$,

$$
\begin{aligned}
M_{t}^{u}\left(r_{T} \omega\right)= & -\left(M^{u}\right)_{(T-t)-}^{T-}(\omega)-2\left(N^{u}\right)_{T-t}^{T}(\omega), \\
N_{t}^{u}\left(r_{T} \omega\right)= & \left(N^{u}\right)_{T-t}^{T}(\omega), \\
\left(f * M^{u}\right)_{t}\left(r_{T} \omega\right)= & -\left(f * M^{u}\right)_{(T-t)-}^{T-}(\omega)-2\left(f * N^{u}\right)_{T-t}^{T}(\omega) \\
& -\sum_{T-t \leq s<T} f\left(X_{s}(\omega)\right) \\
& -f\left(X_{s-}(\omega)\right)\left(u\left(X_{s}(\omega)\right)-u\left(X_{s-}(\omega)\right)\right), \\
\left(f * N^{u}\right)_{t}\left(r_{T} \omega\right)= & \left(f * N^{u}\right)_{T-t}^{T}(\omega) .
\end{aligned}
$$

Under (5.14), each coordinate function $u_{i}(x):=x_{i}(i=1,2, \ldots, d)$ satisfies (5.15). We set $M_{t}:=\left(M_{t}^{u_{1}}, \ldots, M_{t}^{u_{d}}\right)$, and we set $N_{t}:=\left(N_{t}^{u_{1}}, \ldots, N_{t}^{u_{d}}\right)$. Then we have a Fukushima decomposition by [13, Theorem 4.2] (see also [14, Theorem 1.2]):

$$
X_{t}-X_{0}=M_{t}+N_{t} \quad \text { for all } t \in[0, \zeta[
$$

$\mathbf{P}_{x^{-}}$a.s. for q.e. $x \in \mathbb{R}^{d}$; and a Lyons-Zheng decomposition by Corollary 5.3:

$$
X_{t}-X_{0}=\frac{1}{2} M_{t}-\frac{1}{2}\left(M_{T-} \circ r_{T}-M_{(T-t)-} \circ r_{T}\right) \quad \text { for all } t \in[0, T]
$$

$\mathbf{P}_{x^{-}}$a.s. on $\{T<\zeta\}$ for q.e. $x \in \mathbb{R}^{d}$.

\section{§6. Proofs of Theorems 5.1 and 5.2}

Recall that $\Omega$ consists of rcll paths. First we show that any PCAF can be regarded as a continuous reversible PrAFS.

LEMma 6.1. Every PCAF A can be regarded as a continuous reversible $\operatorname{PrAFS}$ with reversible defining sets $\left(\Xi_{t}\right)_{t>0}$ and for $\omega \in \Xi_{T}$ with $T<\zeta(\omega)$, $A_{t}\left(r_{T} \omega\right)=A_{T-t}^{T}(\omega)$ for all $t \in[0, T]$. If the Revuz measure $\mu$ of $A$ has finite total mass, then $A$ can be taken to be a finite continuous reversible PrAFS. Moreover, $A$ can be extended to be a continuous PrAF with some defining sets $\Xi_{t}^{-}\left(\supset \Xi_{t}\right), \Xi^{-}(\supset \Xi)$, and $\widehat{A}$ defined as in (4.1) is a PrAF* with defining sets $\widehat{\Xi}_{t}:=r_{t}^{-1}\left(\Xi_{t}^{-}\right), \widehat{\Xi}:=\bigcap_{t>0} \widehat{\Xi}_{t}$. 


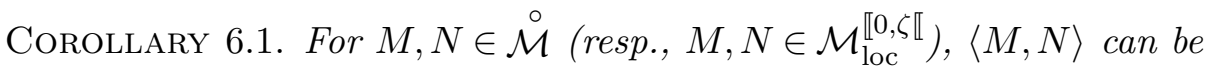
regarded as a finite continuous (resp., continuous) reversible PrAFS having a family of reversible defining sets $\left(\Xi_{t}\right)_{t>0}$, and for $\omega \in \Xi_{T}$ with $T<\zeta(\omega)$, $\langle M, N\rangle_{t}\left(r_{T} \omega\right)=\langle M, N\rangle_{T-t}^{T}(\omega)$ for all $t \in[0, T]$. Moreover, $\langle M, N\rangle$ can be extended to be a continuous $\operatorname{Pr} A F$ with some defining sets $\Xi_{t}^{-}\left(\supset \Xi_{t}\right), \Xi^{-}(\supset$ $\Xi)$, and $\langle\widehat{M, N}\rangle$ defined as in (4.1) is a PrAF* with defining sets $\widehat{\Xi}_{t}:=$ $r_{t}^{-1}\left(\Xi_{t}^{-}\right), \widehat{\Xi}:=\bigcap_{t>0} \widehat{\Xi}_{t}$.

Proof of Lemma 6.1. The last assertion follows from Lemma 4.3 with Remark 4.3. So we prove only the first assertion of Lemma 6.1. First, we suppose that $A$ is a PCAF whose Revuz measure $\mu$ belongs to $S_{0}$. We can reconstruct $A$ as a PrAFS having reversible defining sets in the following way. As in [8, proof of Theorem 5.5.1], let $u$ be a nonnegative finite Borel $\mathcal{E}$-quasicontinuous $\mathfrak{m}$-version of $U_{1} \mu$ such that $n R_{n+1} u(x) \uparrow u(x), n \rightarrow \infty$ for $x \in E \backslash N_{3}$, and $u(x)=0 x \in N_{3}$ for some properly exceptional set. We set $A_{t}^{n}(\omega):=\int_{0}^{t} g_{n}\left(X_{s}(\omega)\right) \mathrm{d} s$, and set $\tilde{A}_{t}^{n}(\omega):=\int_{0}^{t} e^{-s} \mathrm{~d} A_{s}^{n}(\omega)$ for $\omega \in \Omega$, where $g_{n}(x):=n\left(u(x)-n R_{n+1} u(x)\right)(\geq 0), x \in E \backslash N_{3}$, and $g_{n}(x):=0, x \in N_{3}$. In view of [20, Proposition $4.22(\mathrm{iv})]$, there exists an exceptional set $N_{1}$ such that

$$
\mathbf{P}_{x}\left(\{s \in] 0, \zeta\left[\mid X_{s} \neq X_{s-}\right\} \text { is at most countable }\right)=1
$$

for $x \in E \backslash N_{1}$. Taking a common properly exceptional set $N$ including $N_{3}$ and $N_{1}$, we can set

$$
\begin{aligned}
\Omega_{t}:= & \left\{\omega \in \Omega \mid X_{s}(\omega) \in E \backslash N \text { for } s \in\right] 0, t[\text { and } \\
& \left.X_{s-}(\omega) \in E \backslash N \text { for } s \in\right] 0, t[\} .
\end{aligned}
$$

Then $\Omega_{t} \in \mathscr{F}_{t}$ is an $r_{t}$-reversible set. Since $N$ is properly exceptional, we have $\mathbf{P}_{x}\left(\Omega_{t}\right)=1$ for $x \in E \backslash N$. Further, we set

$$
\begin{aligned}
\Xi_{t}\left(A^{n}\right):= & \left\{\omega \in \Omega_{t} \mid \int_{0}^{t} g_{n}\left(X_{s}(\omega)\right) \mathrm{d} s<\infty\right. \text { and } \\
& \left.\{s \in] 0, t\left[\mid X_{s}(\omega) \neq X_{s-}(\omega)\right\} \text { is countable }\right\} .
\end{aligned}
$$

Then $A^{n}$ is a finite continuous PrAFS with the reversible defining set $\Xi_{t}\left(A^{n}\right)$ of $A^{n}$, and for $\omega \in \Xi_{T}\left(A^{n}\right)$ with $T<\zeta(\omega), A_{t}^{n}\left(r_{T} \omega\right)=\left(A^{n}\right)_{T-t}^{T}(\omega)$ for $t \in$ $[0, T]$. We observe that the (pre-)t-equivalence between $\omega_{1}$ and $\omega_{2}$ together with $\omega_{1} \in \Xi_{t}\left(A^{n}\right)$ implies that $\omega_{2} \in \Xi_{t}\left(A^{n}\right)$. In the same way as [8, proof of Theorem 5.2.2], there exists a subsequence $\left\{n_{k}\right\}$ such that $A^{n_{k}}$ converges 
uniformly on each compact interval $\mathbf{P}_{x}$-a.s. for $x \in E \backslash \tilde{N}$, where $\tilde{N}$ is an adequate properly exceptional set. Note that uniform convergence for $\tilde{A}^{n}$ is equivalent to that for $A^{n}$, because $\tilde{A}_{t}^{n}=e^{-t} A_{t}^{n}+\int_{0}^{t} e^{-s} A_{s}^{n} \mathrm{~d} s$ and $A_{t}^{n}=$ $e^{t} \tilde{A}_{t}^{n}-\int_{0}^{t} e^{s} \tilde{A}_{s}^{n} \mathrm{~d} s$. We further set

$$
\Xi_{t}(A):=\left\{\omega \in \bigcap_{k=1}^{\infty} \Xi_{t}\left(A^{n_{k}}\right) \mid A^{n_{k}}(\omega) \text { converges uniformly on }[0, t]\right\},
$$

and we set $A_{t}(\omega):=\varliminf_{k \rightarrow \infty} A_{t}^{n_{k}}(\omega)$ for $\omega \in \Omega$. Then, by Lemma $4.4, A$ is a PrAFS having the reversible defining set $\Xi_{t}(A)$, and for $\omega \in \Xi_{T}(A)$ with $T<\zeta(\omega), A_{t}\left(r_{T} \omega\right)=A_{T-t}^{T}(\omega)$ for $t \in[0, T]$. Next we prove the general case.

Let $A$ be a PCAF with Revuz measure $\mu \in S$. Then there exists an $\mathcal{E}$-nest $\left\{F_{n}\right\}$ of closed sets such that $\mathbf{1}_{F_{n}} \mu \in S_{0}$. Let $A^{(n)}$ be a PCAF which is also a finite continuous PrAFS having a reversible defining set $\Xi_{t}\left(A^{(n)}\right)$ with an exceptional set $N_{n}$ whose Revuz measure is $\mathbf{1}_{F_{n}} \mu \in S_{0}$. We know that for $n<\ell$ there is an exceptional set $N_{n, \ell}$ such that $A_{t}^{(n)}=A_{t}^{(\ell)}$ for all $t<\tau_{F_{n}}$, $\mathbf{P}_{x^{-}}$a.s. for $x \in E \backslash N_{n, \ell}$. Note that

$$
\mathbf{P}_{x}\left(\lim _{n \rightarrow \infty} \sigma_{E \backslash F_{n}} \geq \zeta\right)=\mathbf{P}_{x}\left(\lim _{n \rightarrow \infty} \widehat{\sigma}_{E \backslash F_{n}} \geq \zeta\right)=1
$$

holds for $x \in E \backslash N_{0}$ for some properly exceptional set $N_{0}$ in view of [8, Theorem A.2.3].

Choose a properly exceptional set $N$ containing all $N_{n}, n \in \mathbb{N} \cup\{0\}$ and $N_{n, \ell}, n, \ell \in \mathbb{N}$ with $n<\ell$. We set

$$
\begin{aligned}
\Xi_{t}(A):= & \left\{\omega \in \Omega_{t} \cap \bigcap_{n=1}^{\infty} \Xi_{t}\left(A^{(n)}\right) \mid \text { there exists } n \in \mathbb{N}\right. \text { such that } \\
& t<\sigma_{E \backslash F_{n}}(\omega) \wedge \widehat{\sigma}_{E \backslash F_{n}}(\omega), A_{s}^{(n)}(\omega)=A_{s}^{(\ell)}(\omega) \\
& \text { holds for all } s \in[0, t] \text { and } n<\ell\}
\end{aligned}
$$

where $\Omega_{t}$ is the set for $N$ as in (6.1), and for $\omega \in \Xi_{T}(A)$ we reset $A_{t}(\omega):=$ $A_{t}^{(n)}(\omega), t \in[0, T]$ if $\sigma_{E \backslash F_{n-1}}(\omega) \leq t<\sigma_{E \backslash F_{n}}(\omega), n \in \mathbb{N}$, and $A_{t}(\omega):=$ $A_{\sigma(\omega)-}(\omega)$ if $t \geq \sigma(\omega):=\lim _{n \rightarrow \infty} \sigma_{E \backslash F_{n}}(\omega)$. Note that, for $\omega \in \Xi_{t}(Y), t<$ $\sigma_{E \backslash F_{n}}(\omega)$ (resp., $t<\widehat{\sigma}_{E \backslash F_{n}}(\omega)$ ) implies that $t<\widehat{\sigma}_{E \backslash F_{n}}\left(r_{t} \omega\right)$ (resp., $t<$ $\left.\sigma_{E \backslash F_{n}}\left(r_{t} \omega\right)\right)$. Then $A$ is a continuous PrAFS having the reversible defining set $\Xi_{t}(A)$, and for $\omega \in \Xi_{T}(A)$ with $T<\zeta(\omega), A_{t}\left(r_{T} \omega\right)=A_{T-t}^{T}(\omega)$ for $t \in[0, T]$. 
Finally, suppose that the Revuz measure $\mu$ of $A$ has total mass. Then $\mathbf{E}_{x}\left[A_{t}\right]<\infty$ for each $t \in\left[0, \infty\right.$ [ for q.e. $x \in E$; hence, $\mathbf{P}_{x}\left(A_{t}<\infty\right.$ for all $t \in$ $\left[0, \infty[)=1\right.$ for q.e. $x \in E$. So $\widetilde{\Xi}_{t}(A):=\left\{\omega \in \Xi_{t}(A) \mid A_{t}(\omega)<\infty\right\}$ forms a reversible defining set for $A$. This implies that $A$ is a finite continuous PrAFS.

Next we show that any MAF of finite energy and CAF of zero energy appearing in the Fukushima decomposition can be regarded as finite rcll reversible PrAFSs with reversible defining sets.

Lemma 6.2. Take $u \in \mathcal{F}_{e}$, let $M^{u} \in \stackrel{\circ}{\mathcal{M}}$ be the $M A F$ of finite energy, and let $N^{u} \in \mathcal{N}_{c}$ be the CAF of zero energy appearing in the Fukushima decomposition for $u$. Then $M^{u}$ (resp., $N^{u}$ ) can be regarded as a finite rcll (resp., finite continuous) reversible PrAFS with a family of common reversible defining sets $\left(\Xi_{t}(u)\right)_{t>0}$ such that, for $\omega \in \Xi_{T}(u)$ with $T<\zeta(\omega)$, $M_{t}^{u}\left(r_{T} \omega\right)=-\left(M^{u}\right)_{(T-t)-}^{T-}(\omega)-2\left(N^{u}\right)_{T-t}^{T}(\omega)$ and $N_{t}^{u}\left(r_{T} \omega\right)=\left(N^{u}\right)_{T-t}^{T}(\omega)$. Moreover, for $f, u \in \mathcal{F}_{e}$ with $f \in L^{2}\left(E ; \mu_{\left\langle M^{u}\right\rangle}\right), f * M^{u}\left(\right.$ resp., $\left.\Gamma\left(f * M^{u}\right)\right)$ can be regarded as a finite rcll (resp., finite continuous) reversible PrAFS with a family of common reversible defining sets $\left(\Xi_{t}(f, u)\right)_{t>0}$ such that, for $\omega \in \Xi_{T}(f, u)$ with $T<\zeta(\omega)$,

$$
\begin{aligned}
\left(f * M^{u}\right)_{t}\left(r_{T} \omega\right)= & -\left(f * M^{u}\right)_{(T-t)-}^{T-}(\omega) \\
& -\left[M^{f}, M^{u}\right]_{(T-t)-}^{T-}(\omega)-2\left(f * \Gamma\left(M^{u}\right)\right)_{T-t}^{T}(\omega)
\end{aligned}
$$

and $\Gamma\left(f * M^{u}\right)_{t}\left(r_{T} \omega\right)=\Gamma\left(f * M^{u}\right)_{T-t}^{T}(\omega)$.

Corollary 6.2. Take $M \in \stackrel{\circ}{\mathcal{M}}$, and take $f \in \mathcal{F}_{e} \cap L^{2}\left(E ; \mu_{\langle M\rangle}\right)$. Then $\Gamma(M), f * \Gamma(M), \Gamma(f * M)$, and $\left\langle M^{f, c}+M^{f, j}, M^{c}+M^{j}+K\right\rangle$ can be regarded as finite continuous reversible PrAFSs with a family of common reversible defining sets $\left(\Xi_{t}(u)\right)_{t>0}$ such that, for $\omega \in \Xi_{T}(u)$ with $T<\zeta(\omega)$ and $t \in[0, T], \quad \Gamma(M)_{t}\left(r_{T} \omega\right)=\Gamma(M)_{T-t}^{T}(\omega)$ and $(f * \Gamma(M))_{t}\left(r_{T} \omega\right)=$ $(f * \Gamma(M))_{T-t}^{T}(\omega)$.

Proof. Applying Lemma 6.2 to (3.2), we first obtain the assertion for $\Gamma(M)$. The second assertion is clear from the first assertion with (3.4) and Corollary 6.1.

Proof of Lemma 6.2. We prove only the latter assertion. The proof of the former one is easy. We first prove for the case $u=R_{1} g$ with Borel 
measurable $g \in L^{2}(E ; \mathfrak{m})$ and $f \in\left(\mathcal{F}_{e}\right)_{b}$. In this case, $N^{u}=\Gamma\left(M^{u}\right)$ is a CAF of locally bounded variation. In particular, from [18, Theorem 3.1], the FiskStratonovich integral

$$
\int_{0}^{t} f\left(X_{s}\right) \circ \mathrm{d} A_{s}^{u}=\int_{0}^{t} f\left(X_{s-}\right) \mathrm{d} A_{s}^{u}+\frac{1}{2}\left[M^{f}, M^{u}\right]_{t}
$$

is a semimartingale on $\left[0, \infty\left[\right.\right.$ under $\mathbf{P}_{x}$ for q.e. $x \in E$. Set $t_{i}^{n}:=i T / 2^{n}$, $i=0,1, \ldots, 2^{n}$. Then $\left\{t_{i}^{n}\right\}$ forms a subdivision of $[0, T]$. For simplicity, we write $t_{i}=t_{i}^{n}$. We may assume that $X_{t_{i}}=X_{t_{i}-}$ (hence, $M_{t_{i}}^{u}=M_{t_{i}-}^{u}$ ), $i=$ $0,1, \ldots, 2^{n}, n \in \mathbb{N}, \mathbf{P}_{x}$-a.s. for q.e. $x \in E$.

By [10, Proposition 4.44 and Theorem 4.47 in Chapter I], we see that

$$
\begin{aligned}
& \sup _{t \in[0, T]}\left|\sum_{i=0}^{2^{n}-1}\left(f\left(X_{t_{i+1} \wedge t}\right)-f\left(X_{t_{i} \wedge t}\right)\right)\left(M_{t_{i+1} \wedge t}^{u}-M_{t_{i} \wedge t}^{u}\right)-\left[M^{f}, M^{u}\right]_{t}\right|, \\
& \sup _{t \in[0, T]}\left|\sum_{i=0}^{2^{n}-1}\left(f\left(X_{t_{i+1} \wedge t}\right)-f\left(X_{t_{i} \wedge t}\right)\right)\left(N_{t_{i+1} \wedge t}^{u}-N_{t_{i} \wedge t}^{u}\right)\right| \\
& \sup _{t \in[0, T]}\left|\sum_{i=0}^{2^{n}-1} f\left(X_{t_{i}}\right)\left(M_{t_{i+1} \wedge t}^{u}-M_{t_{i} \wedge t}^{u}\right)-\int_{0}^{t} f\left(X_{s-}\right) \mathrm{d} M_{s}^{u}\right|
\end{aligned}
$$

and

$$
\sup _{t \in[0, T]}\left|\sum_{i=0}^{2^{n}-1} f\left(X_{t_{i}}\right)\left(N_{t_{i+1} \wedge t}^{u}-N_{t_{i} \wedge t}^{u}\right)-\int_{0}^{t} f\left(X_{s}\right) \mathrm{d} N_{s}^{u}\right|
$$

converge to 0 in $\mathbf{P}_{x}$-probability for q.e. $x \in E$. Note that $s \mapsto f\left(X_{s-}\right)$ is bounded left-continuous. In particular,

$$
t \mapsto \sum_{i=0}^{2^{n}-1} \frac{f\left(X_{t_{i+1} \wedge t}\right)+f\left(X_{t_{i}}\right)}{2}\left(A_{t_{i+1} \wedge t}^{u}-A_{t_{i} \wedge t}^{u}\right)
$$

uniformly converges to $\int_{0}^{t} f\left(X_{s-}\right) d A_{s}^{u}+\frac{1}{2}\left[M^{f}, M^{u}\right]_{t}$ on $[0, T]$ in $\mathbf{P}_{x^{-}}$ probability for q.e. $x \in E$. By way of the usual diagonal argument, we can construct a suitable subsequence $\left\{n_{k}\right\}$ (we write it $\{n\}$ again for simplicity) such that the above Riemann sums uniformly converge on $[0, T] \mathbf{P}_{x^{-a} \text {.s. for }}$ q.e. $x \in E$. Let $\left(\Xi_{t}\right)_{t>0}$ be the common reversible defining sets of $M^{u}, N^{u}$, and $\left\langle M^{f, c}+M^{f, j}, M^{u, c}+M^{u, j}\right\rangle$, which admits an exceptional set. Let $\Omega_{T}$ be 
the set analogously defined as in (6.1) for an adequate properly exceptional set $N$ containing all exceptional sets that appeared so far in this proof. Set

$$
\begin{aligned}
\Xi_{T}(f, u):= & \left\{\omega \in \Omega_{T} \cap \Xi_{T}(u) \mid\right. \\
& \sum_{i=0}^{2^{n}-1} f\left(X_{t_{i+1} \wedge t}(\omega)-f\left(X_{t_{i} \wedge t}(\omega)\right)\left(M_{t_{i+1} \wedge t}^{u}(\omega)-M_{t_{i} \wedge t}^{u}(\omega)\right),\right. \\
& \sum_{i=0}^{2^{n}-1} f\left(X_{t_{i+1} \wedge t}(\omega)-f\left(X_{t_{i} \wedge t}(\omega)\right)\right)\left(N_{t_{i+1} \wedge t}^{u}(\omega)-N_{t_{i} \wedge t}^{u}(\omega)\right), \\
& \sum_{i=0}^{2^{n}-1} f\left(X_{t_{i}}(\omega)\right)\left(M_{t_{i+1} \wedge t}^{u}(\omega)-M_{t_{i} \wedge t}^{u}(\omega)\right), \text { and } \\
& \sum_{i=0}^{2^{n}-1} f\left(X_{t_{i}}(\omega)\right)\left(N_{t_{i+1} \wedge t}^{u}(\omega)-N_{t_{i} \wedge t}^{u}(\omega)\right)
\end{aligned}
$$

uniformly converge on $[0, T]$;

$M_{t}^{u}(\omega)\left(\right.$ resp., $\left.M_{t-}^{u}(\omega)\right)$ is right-continuous

(resp., left-continuous); and $N_{t}^{u}(\omega)$ is continuous on $\left.[0, T]\right\}$.

Then $\left(\Xi_{T}(f, u)\right)_{T>0}$ is a family of reversible defining sets. Indeed, by setting $i_{t}:=\left[2^{n} t / T\right]$ and $j_{t}:=2^{n}-i_{t}$ and noting that $t_{j}=T-t_{2^{n}-j}$, we see that $t_{i_{t}} \leq t<t_{i_{t}+1}$ and $t_{j_{t}-1}<T-t \leq t_{j_{t}}$. We then have that, for $\omega \in \Xi_{T}$ with $T<\zeta(\omega)$,

$$
\begin{aligned}
& \left(\sum_{i=0}^{2^{n}-1} f\left(X_{t_{i}}(\omega)\right)\left(M_{t_{i+1} \wedge t}^{u}(\omega)-M_{t_{i} \wedge t}^{u}(\omega)\right)\right) \circ r_{T} \\
& =\sum_{i=0}^{2^{n}-1} f\left(X_{\left(T-t_{i}\right)-}(\omega)\right)\left(M_{t_{i+1} \wedge t}^{u}\left(r_{T} \omega\right)-M_{t_{i} \wedge t}^{u}\left(r_{T} \omega\right)\right) \\
& =\sum_{i=0}^{i_{t}-1} f\left(X_{\left(T-t_{i}\right)-}(\omega)\right)\left(M_{t_{i+1}}^{u}\left(r_{T} \omega\right)-M_{t_{i}}^{u}\left(r_{T} \omega\right)\right) \\
& \quad+f\left(X_{\left(T-t_{i_{t}}\right)-}(\omega)\right)\left(M_{t}^{u}\left(r_{T} \omega\right)-M_{t_{i_{t}}}^{u}\left(r_{T} \omega\right)\right) \\
& =\sum_{i=0}^{i_{t}-1} f\left(X_{\left(T-t_{i}\right)-}(\omega)\right)\left(M_{\left(T-t_{i+1}\right)-}^{u}(\omega)-M_{\left(T-t_{i}\right)-}^{u}(\omega)\right)
\end{aligned}
$$




$$
\begin{aligned}
& +2 \sum_{i=0}^{i_{t}-1} f\left(X_{\left(T-t_{i}\right)-}(\omega)\right)\left(N_{T-t_{i+1}}^{u}(\omega)-N_{T-t_{i}}^{u}(\omega)\right) \\
& +f\left(X_{\left(T-t_{i_{t}}\right)-}(\omega)\right)\left(M_{(T-t)-}^{u}(\omega)-M_{\left(T-t_{i_{t}}\right)-}^{u}(\omega)\right) \\
& +2 f\left(X_{\left(T-t_{i_{t}}\right)-}(\omega)\right)\left(N_{T-t}^{u}(\omega)-N_{T-t_{i_{t}}}^{u}(\omega)\right) \\
& =\sum_{j=j_{t}}^{2^{n}-1} f\left(X_{t_{j+1}-}(\omega)\right)\left(M_{t_{j}-}^{u}(\omega)-M_{t_{j+1}-}^{u}(\omega)\right) \\
& +2 \sum_{j=j_{t}}^{2^{n}-1} f\left(X_{t_{j+1}-}(\omega)\right)\left(N_{t_{j}}^{u}(\omega)-N_{t_{j+1}}^{u}(\omega)\right) \\
& +f\left(X_{t_{j_{t}}-}(\omega)\right)\left(M_{(T-t)-}^{u}(\omega)-M_{t_{j_{t}}}^{u}(\omega)\right) \\
& +2 f\left(X_{t_{j_{t}}-}(\omega)\right)\left(N_{(T-t)}^{u}(\omega)-N_{t_{j_{t}}}^{u}(\omega)\right) \\
& =\sum_{j=0}^{2^{n}-1} f\left(X_{t_{j+1}-}(\omega)\right)\left(M_{t_{j}-}^{u}(\omega)-M_{t_{j+1}-}^{u}(\omega)\right) \\
& +2 \sum_{j=0}^{2^{n}-1} f\left(X_{t_{j+1}-}(\omega)\right)\left(N_{t_{j}}^{u}(\omega)-N_{t_{j+1}}^{u}(\omega)\right) \\
& -\sum_{j=0}^{2^{n}-1} f\left(X_{t_{j+1} \wedge(T-t)-}(\omega)\right)\left(M_{t_{j} \wedge(T-t)-}^{u}(\omega)-M_{t_{j+1} \wedge(T-t)-}^{u}(\omega)\right) \\
& -2 \sum_{j=0}^{2^{n}-1} f\left(X_{t_{j+1} \wedge(T-t)-}(\omega)\right)\left(N_{t_{j} \wedge(T-t)}^{u}(\omega)-N_{t_{j+1} \wedge(T-t)}^{u}(\omega)\right) \\
& +\left(f\left(X_{t_{j_{t}}-}(\omega)\right)-f\left(X_{(T-t)-}(\omega)\right)\right)\left(M_{t_{j_{t}-1}-}^{u}(\omega)-M_{(T-t)-}^{u}(\omega)\right) \\
& +2\left(f\left(X_{t_{j_{t}}}(\omega)\right)-f\left(X_{(T-t)-}(\omega)\right)\right)\left(N_{t_{j_{t}-1}}^{u}(\omega)-N_{T-t}^{u}(\omega)\right),
\end{aligned}
$$

which uniformly converges to

$$
-\left(f * M^{u}\right)_{(T-t)-}^{T-}(\omega)-2\left(f * N^{u}\right)_{T-t}^{T}(\omega)-\left[M^{f}, M^{u}\right]_{(T-t)-}^{T-}(\omega) .
$$

We will explain why the last two terms uniformly converge to 0 . Since $t \mapsto M_{t-}^{u}(\omega)\left(M_{0-}(\omega):=M_{0}^{u}(\omega)=0\right)$ is left-continuous on $[0, T]$, we can extend $M_{t-}(\omega)$ as a left-continuous function on $\left[0, \infty\left[\right.\right.$ by putting $M_{s-}^{u}(\omega):=$ 
$M_{t-}^{u}(\omega)$ for $s \geq t$. Then we see that $M_{t-}^{u}(\omega)$ is uniformly left-continuous on $[0, T]$ in the following sense: for any $\varepsilon>0$, there exists $\delta>0$ such that

$$
\sup _{t \in[0, T]} \sup _{s \in] t-\delta, t[\cap[0, T]}\left|M_{t-}^{u}(\omega)-M_{s-}^{u}(\omega)\right|<\varepsilon .
$$

The proof of this uniform left-continuity is the same as in the proof of the uniform continuity of continuous functions on compact sets. Noting that $0<(T-t)-t_{j_{t}-1}<T / 2^{n}$ and $0<t_{j_{t}}-(T-t)<T / 2^{n}$, the last two terms in the right-hand side uniformly converge to 0 with respect to $t \in[0, T]$.

The uniform convergences of

$$
\begin{aligned}
& t \mapsto\left(\sum_{i=0}^{2^{n}-1} f\left(X_{t_{i}}(\omega)\right)\left(N_{t_{i+1} \wedge t}^{u}(\omega)-N_{t_{i} \wedge t}^{u}(\omega)\right)\right) \circ r_{T}, \\
& t \mapsto\left(\sum_{i=0}^{2^{n}-1}\left(f\left(X_{t_{i+1} \wedge t}(\omega)\right)-f\left(X_{t_{i} \wedge t}(\omega)\right)\right)\left(M_{t_{i+1} \wedge t}^{u}(\omega)-M_{t_{i} \wedge t}^{u}(\omega)\right)\right) \circ r_{T}, \\
& t \mapsto\left(\sum_{i=0}^{2^{n}-1}\left(f\left(X_{t_{i+1} \wedge t}(\omega)\right)-f\left(X_{t_{i} \wedge t}(\omega)\right)\right)\left(N_{t_{i+1} \wedge t}^{u}(\omega)-N_{t_{i} \wedge t}^{u}(\omega)\right)\right) \circ r_{T}
\end{aligned}
$$

are similarly confirmed. Hence we, have the $r_{T}$-reversibility of $\Xi_{T}(f, u)$. Then, for such $\omega \in \Xi_{T}(f, u)$ with $T<\zeta(\omega)$, we have (6.2) for all $t \in[0, T]$. Therefore, we obtain the desired formula (5.1).

Next we prove the case for $u \in \mathcal{F}, f \in\left(\mathcal{F}_{e}\right)_{b}$. Taking $u_{n}=R_{1} g_{n}, g_{n}:=$ $n\left(u-n R_{n+1} u\right) \in L^{2}(E ; \mathfrak{m}) \cap \mathscr{B}(E)$. We know that $f * M^{u_{n}} \in \stackrel{\circ}{\mathcal{M}}$ is econvergent to $f * M^{u}$ in $\stackrel{\circ}{\mathcal{M}}$. Moreover, let $u_{n, k}:=u_{n}-u_{k}$, and for $\nu \in S_{00}$, we see that

$$
\begin{aligned}
& \mathbf{E}_{\nu}\left[\sup _{t \in[0, T]}\left|\sum_{0<s \leq t}\left(f\left(X_{s}\right)-f\left(X_{s-}\right)\right)\left(u_{n, k}\left(X_{s}\right)-u_{n, k}\left(X_{s-}\right)\right)\right|\right] \\
& \leq \mathbf{E}_{\nu}\left[\sum_{0<s \leq T}\left(f\left(X_{s}\right)-f\left(X_{s-}\right)\right)^{2}\right]^{1 / 2} \\
& \quad \times \mathbf{E}_{\nu}\left[\sum_{0<s \leq T}\left(u_{n, k}\left(X_{s}\right)-u_{n, k}\left(X_{s-}\right)\right)^{2}\right]^{1 / 2} \\
& \leq \mathbf{E}_{\nu}\left[\left\langle M^{f, d}\right\rangle_{T}\right]^{1 / 2} \mathbf{E}_{\nu}\left[\left\langle M^{u_{n, k}, d}\right\rangle_{T}\right]^{1 / 2} \\
& \leq(1+T)\left\|U_{1} \nu\right\|_{\infty} \mathbf{e}\left(M^{f, d}\right)^{1 / 2} \mathbf{e}\left(M^{u_{n, k}, d}\right)^{1 / 2} \rightarrow 0 \quad \text { as } n, k \rightarrow \infty
\end{aligned}
$$


Then one can construct a common subsequence $\left\{n_{k}\right\}$ such that $f * M^{u_{n_{k}}}$ (resp., $\left[M^{f}, M^{u_{n}}\right], f * \Gamma\left(M^{u_{n}}\right)$ ) uniformly converges to $f * M^{u}$ (resp., $\left.\left[M^{f}, M^{u}\right], f * M^{u}\right)$ on $[0, T], \mathbf{P}_{x^{-}}$a.s. for q.e. $x \in E$. We can take a common subsequence so that both convergences hold. Set

$$
\begin{aligned}
\Xi_{T}:= & \left\{\omega \in \bigcap_{k=1}^{\infty} \Xi_{T}\left(f, u_{n_{k}}\right) \cap \Xi_{T}\left(f * \Gamma\left(M^{u_{n_{k}}}\right)\right) \mid\left(f * M^{u_{n_{k}}}\right) .(\omega),\right. \\
& \left.\quad f * \Gamma\left(M^{u_{n_{k}}}\right) .(\omega) \text { and }\left[M^{f}, M^{u_{n_{k}}}\right] .(\omega) \text { uniformly converge on }[0, T]\right\},
\end{aligned}
$$

where $\Xi_{t}\left(f * \Gamma\left(M^{u_{n_{k}}}\right)\right)$ is the reversible defining set of $f * \Gamma\left(M^{u_{n_{k}}}\right)$. Then $\left(\Xi_{T}\right)_{T>0}$ is a family of reversible defining sets. Indeed, by use of Corollary 6.1, we have $\Gamma\left(f * M^{u_{n_{k}}}\right)\left(r_{T} \omega\right)=\Gamma\left(f * M^{u_{n_{k}}}\right)_{T-t}^{T}(\omega)$ and

$$
\begin{aligned}
f * M_{t}^{u_{n_{k}}}\left(r_{T} \omega\right)= & -\left(f * M^{u_{n_{k}}}\right)_{(T-t)-}^{T-}(\omega) \\
& -2\left(f * \Gamma\left(M^{u_{n_{k}}}\right)\right)_{T-t}^{T}(\omega)-\left[M^{f}, M^{u_{n_{k}}}\right]_{(T-t)-}^{T-}(\omega),
\end{aligned}
$$

which means that $r_{T} \omega \in \Xi_{T}$ and that (6.2) holds for $\omega \in \Xi_{T}$ with $T<\zeta(\omega)$. The strategy of the proof for the case $f \in\left(\mathcal{F}_{e}\right)_{b}, u \in \mathcal{F}_{e}$ is similar to this proof by $\mathcal{E}$-approximating $u_{n} \in \mathcal{F}$. For general $f \in \mathcal{F}_{e} \cap L^{2}\left(E ; \mu_{\left\langle M^{u}\right\rangle}\right)$ with $u \in \mathcal{F}_{e}$, we can approximate $f$ by $f_{n}:=(-n) \vee f \wedge n \in\left(\mathcal{F}_{e}\right)_{b}$. Then $f_{n} * M^{u}$ is e-convergent to $f * M^{u}$. The rest is similar.

Proofs of Theorems 5.1 and 5.2.

(Step 1): Proof of Theorem 5.2 with (5.1) for $M \in \stackrel{\circ}{M}^{d}, f \in \mathcal{F}_{e} \cap$ $L^{2}\left(E ; \mu_{\langle M\rangle}\right)$.

In this case, $M=M^{d}$. For any $\nu \in S_{0}$, we have

$$
\begin{aligned}
\mathbf{E}_{\nu}\left[\sum_{0<s \leq t}\left|\frac{f\left(X_{s}\right)+f\left(X_{s-}\right)}{2} \mathbf{1}_{E \times E} \varphi_{\ell}\left(X_{s-}, X_{s}\right)\right|\right] \\
\leq \frac{1}{2} \mathbf{E}_{\nu}\left[\sum_{0<s \leq t}\left|\left(f\left(X_{s}\right)-f\left(X_{s-}\right)\right) \mathbf{1}_{E \times E} \varphi_{\ell}\left(X_{s-}, X_{s}\right)\right|\right] \\
\quad+\mathbf{E}_{\nu}\left[\sum_{0<s \leq t}\left|f\left(X_{s-}\right) \mathbf{1}_{E \times E} \varphi_{\ell}\left(X_{s-}, X_{s}\right)\right|\right] \\
\leq \frac{1}{2} \mathbf{E}_{\nu}\left[\sum_{0<s \leq t}\left(f\left(X_{s}\right)-f\left(X_{s-}\right)\right)^{2}\right]^{1 / 2} \mathbf{E}_{\nu}\left[\sum_{0<s \leq t} \varphi^{2}\left(X_{s-}, X_{s}\right) \mathbf{1}_{\{s<\zeta\}}\right]^{1 / 2}
\end{aligned}
$$




$$
\begin{aligned}
& +\ell \mathbf{E}_{\nu}\left[\int_{0}^{t}\left|f\left(X_{s}\right)\right| \int_{E} \varphi^{2}\left(X_{s}, y\right) N\left(X_{s}, \mathrm{~d} y\right) \mathrm{d} H_{s}\right] \\
\leq & \frac{1}{2} \mathbf{E}_{\nu}\left[\left\langle M^{f, j}\right\rangle_{t}\right]^{1 / 2} \mathbf{E}_{\nu}\left[\left\langle M^{j}\right\rangle_{t}\right]^{1 / 2}+\ell \mathbf{E}_{\nu}\left[\left\langle|f| * M^{j}, M^{j}\right\rangle_{t}\right]<\infty
\end{aligned}
$$

and, by [8, Lemma 5.1.9],

$$
\begin{aligned}
\mathbf{E}_{\nu}\left[\sum_{0<s \leq t}\left|\frac{f\left(X_{s}\right)+f\left(X_{s-}\right)}{2} \mathbf{1}_{E \times E} \overline{\varphi_{\ell}}\left(X_{s-}, X_{s}\right)\right|\right] \\
\leq \frac{1}{2} \mathbf{E}_{\nu}\left[\sum_{0<s \leq t}\left|\left(f\left(X_{s-}\right)-f\left(X_{s}\right)\right) \mathbf{1}_{E \times E} \overline{\varphi_{\ell}}\left(X_{s-}, X_{s}\right)\right|\right] \\
\quad+\mathbf{E}_{\nu}\left[\sum_{0<s \leq t}\left|f\left(X_{s}\right) \mathbf{1}_{E \times E} \overline{\varphi_{\ell}}\left(X_{s-}, X_{s}\right)\right|\right] \\
\leq \frac{1+t}{2}\left\|U_{1} \nu\right\|_{\infty} \mathbf{e}\left(M^{f, j}\right)^{1 / 2} \mathbf{e}\left(M^{j}\right)^{1 / 2}+\ell(1+t)\left\|U_{1} \nu\right\|_{\infty} \mathbf{e}\left(|f| * M^{j}, M^{j}\right) \\
<\infty
\end{aligned}
$$

By [13, Lemma 4.1(2)], we have that $\Gamma\left(K^{\ell}\right)_{t}=f * \Gamma\left(K^{\ell}\right)_{t}=0$ holds for all $t \in$ $\left[0, \zeta\left[\mathbf{P}_{x^{-}}\right.\right.$a.s. for q.e. $x \in E$. In particular, $\Gamma\left(K^{\ell}\right)$ and $f * \Gamma\left(K^{\ell}\right)$ are PCAFs by setting $\Gamma\left(K^{\ell}\right)_{t}=f * \Gamma\left(K^{\ell}\right)_{t}=0$ for $t \geq \zeta$. Hence, they can be regarded as PrAFS with the common reversible defining set $\Xi_{T}\left(\Gamma\left(K^{\ell}\right)\right) \cap \Xi_{T}\left(f * \Gamma\left(K^{\ell}\right)\right)$ in view of Lemma 6.1 ; in particular, $\omega \in \Xi_{T}\left(\Gamma\left(K^{\ell}\right)\right) \cap \Xi_{T}\left(f * \Gamma\left(K^{\ell}\right)\right)$ implies that $\Gamma\left(K^{\ell}\right)_{t}(\omega)=f * \Gamma\left(K^{\ell}\right)_{t}(\omega)=0$ for all $t \in[0, T]$. Then

$$
\begin{aligned}
\Xi_{T}(f, \ell):= & \left\{\omega \in \Omega_{T} \cap \Xi_{T}\left(\Gamma\left(f *\left(M^{d, \ell}+K^{\ell}\right)\right)\right) \cap \Xi_{T}\left(\left\langle M^{f}, M^{d, \ell}+K^{\ell}\right\rangle\right)\right. \\
& \cap \Xi_{T}\left(\Gamma\left(K^{\ell}\right)\right) \cap \Xi_{T}\left(f * \Gamma\left(K^{\ell}\right)\right) \mid \\
& \left.\{s \in] 0, T] \mid X_{s}(\omega) \neq X_{s-}(\omega)\right\} \text { is countable, } \\
& t \mapsto \sum_{0<s \leq t} f\left(X_{s-}(\omega)\right) \mathbf{1}_{E \times E} \varphi_{\ell}\left(X_{s-}(\omega), X_{s}(\omega)\right), \text { and } \\
& t \mapsto \sum_{0<s \leq t} f\left(X_{s-}(\omega)\right) \mathbf{1}_{E \times E} \overline{\varphi_{\ell}}\left(X_{s-}(\omega), X_{s}(\omega)\right)
\end{aligned}
$$

are absolutely uniformly convergent on $[0, T]\}$

is an $r_{T}$-reversible defining set of $f *\left(M^{d, \ell}+K^{\ell}\right), f * \Gamma\left(M^{d, \ell}+K^{\ell}\right)$, and $\left[M^{f}, M^{d, \ell}+K^{\ell}\right]$. Here $\Omega_{T}$ is analogously defined as in (6.1) for an adequate 
properly exceptional set $N$ containing all exceptional sets that appeared so far in this proof. Then for $\omega \in \Xi_{T}(f, \ell)$ with $T<\zeta(\omega)$ and $t \in[0, T]$, we have $f * \Gamma\left(K^{\ell}\right)_{t}(\omega)=0, r_{T} \omega \in \Xi_{T}(f, \ell)$, and

$$
\begin{aligned}
\left(f * M^{d, \ell}\right)_{t}\left(r_{T} \omega\right)= & -\left(f *\left(M^{d, \ell}+K^{\ell}\right)\right)_{(T-t)-}^{T-}(\omega)-2\left(f * \Gamma\left(M^{d, \ell}\right)\right)_{T-t}^{T}(\omega) \\
& -\left[M^{f}, M^{d, \ell}+K^{\ell}\right]_{(T-t)-}^{T-}(\omega) .
\end{aligned}
$$

Since $f * M^{d, \ell}$ (resp., $f * K^{\ell}, M^{d, \ell}, K^{\ell}$ ) is e-convergent to $f * M^{d}$ (resp., $\left.f * K, M^{d}, K\right)$, by [18, Theorem 3.2] there exists a common subsequence $\left\{\ell_{k}\right\}$ such that $f * M^{d, \ell_{k}}, K^{\ell_{k}}$, and $f * \Gamma\left(M^{d, \ell_{k}}\right)$ uniformly converge to $f *$ $M^{d}, f * K$, and $f * \Gamma\left(M^{d}\right)$, and $\left[M^{f}, M^{d, \ell_{k}}+K^{\ell_{k}}\right]$ uniformly converges to $\left[M^{f}, M^{d}+K\right]$ on each compact subinterval of $\left[0, \infty\left[\mathbf{P}_{x^{-}}\right.\right.$a.s. for q.e. $x \in E$. Then for such subsequence $\left\{\ell_{k}\right\}$ we can define the following defining set with an adequate properly exceptional $N$ containing all countable exceptional sets that appeared so far in the proof:

$$
\begin{aligned}
\Xi_{T}\left(f * M^{d}\right):= & \left\{\omega \in \Omega_{T} \cap \bigcap_{k=1}^{\infty} \Xi_{T}\left(f, \ell_{k}\right) \mid \text { all } f *\left(M^{d, \ell_{k}}+K^{\ell_{k}}\right)(\omega),\right. \\
& f * \Gamma\left(M^{d, \ell_{k}}+K^{\ell_{k}}\right)(\omega) \text { and } \\
& {\left.\left[M^{f}, M^{d, \ell_{k}}+K^{\ell_{k}}\right](\omega) \text { uniformly converge on }[0, T]\right\}, }
\end{aligned}
$$

where $\Omega_{T}$ is the set defined for $N$ as in (6.1). We see that $\Xi_{T}\left(f * M^{d}\right)$ is an $r_{T}$-reversible set. Then, for $\omega \in \Xi_{T}\left(f * M^{d}\right)$ with $T<\zeta(\omega)$, we have, for $t \in[0, T], f * \Gamma(K)_{t}(\omega)=0$ and

$$
\begin{aligned}
\left(f * M^{d}\right)_{t}\left(r_{T} \omega\right)= & -\left(f *\left(M^{d}+K\right)\right)_{(T-t)-}^{T-}(\omega) \\
& -2\left(f * \Gamma\left(M^{d}\right)\right)_{T-t}^{T}(\omega)-\left[M^{f}, M^{d}+K\right]_{(T-t)-}^{T-}(\omega),
\end{aligned}
$$

which shows (5.1).

(Step 2): Proof of Theorem 5.2 with (5.1) for $M \in \stackrel{\circ}{\mathcal{M}}^{c}$ and $f \in \mathcal{F}_{e} \cap$ $L^{2}\left(E ; \mu_{\langle M\rangle}\right)$.

In this case, $M=M^{c}$. Consider a subfamily

$$
\stackrel{\circ}{\mathcal{M}}_{0}^{c}:=\left\{f * M^{u, c} \in \stackrel{\circ}{\mathcal{M}}^{c} \mid f \in \mathcal{F} \cap C_{0}(E), u \in \mathcal{F}\right\}
$$


of $\stackrel{\circ}{\mathcal{M}}^{c}$. In view of [8, proof of Lemma 5.6.1], we have that $\stackrel{\circ}{\mathcal{M}}_{0}^{c}$ is dense in $\left(\stackrel{\circ}{\mathcal{M}}^{c}, \mathbf{e}\right)$. Hence, for any $M \in \stackrel{\circ}{\mathcal{M}}^{c}$, we can take an e-convergent sequence $\left\{M^{n}\right\} \subset \dot{\mathcal{M}}_{0}^{c}$ to $M$. Note that $M^{n}=f_{n} * M^{u_{n}, c}$ for some $f_{n} \in \mathcal{F} \cap C_{0}(E)$, $u_{n} \in \mathcal{F}$. Then there exists a subsequence $\left\{n_{k}\right\}$ such that $M_{t}^{n_{k}}$ uniformly converges on $[0, T], \mathbf{P}_{x^{-}}$a.s. for q.e. $x \in E$. By [18, Theorem 3.2], we have that there exists a subsequence $\left\{n_{k}\right\}$ such that $\Gamma\left(M^{n_{k}}\right)_{t}$ uniformly converges to $\Gamma(M)_{t}$ on $[0, T], \mathbf{P}_{x}$-a.s. for q.e. $x \in E$. Take a common subsequence so that both convergences hold. Set

$$
\begin{aligned}
\Xi_{T}\left(f * M^{c}\right):= & \left\{\omega \in \bigcap_{k=1}^{\infty} \Xi_{T}\left(f_{n_{k}}, u_{n_{k}}\right) \cap \Xi_{T}\left(\Gamma\left(M^{n_{k}}\right)\right) \mid M^{n_{k}}(\omega)\right. \text { and } \\
& \left.\Gamma\left(M^{n_{k}}\right)(\omega) \text { uniformly converge on }[0, T]\right\}
\end{aligned}
$$

where $\Xi_{T}\left(f_{n_{k}}, u_{n_{k}}\right)$ is the intersection of the reversible defining sets of $f_{n_{k}}$ * $M^{u_{n_{k}}}$ and $f_{n_{k}} * M^{u_{n_{k}}, d}$, which are defined in the proof of Lemma 6.2 and in the proof for the case that $M \in \stackrel{\circ}{ }^{d}$ and $\Xi_{T}\left(\Gamma\left(M^{n_{k}}\right)\right)$ is the reversible defining set of $\Gamma\left(M^{n_{k}}\right)$. Then $\left(\Xi_{T}\left(f * M^{c}\right)\right)_{T>0}$ is a family of common reversible defining sets of $M$ and $\Gamma(M)$. Indeed, by use of Corollary 6.2, we have $\Gamma\left(M^{n_{k}}\right)\left(r_{T} \omega\right)=\Gamma\left(M^{n_{k}}\right)_{T-t}^{T}(\omega)$ and

$$
\begin{aligned}
M_{t}^{n_{k}}\left(r_{T} \omega\right) & =A_{t}^{n_{k}}\left(r_{T} \omega\right)-\Gamma\left(M^{n_{k}}\right)_{t}\left(r_{T} \omega\right) \\
& =-\left(A^{n_{k}}\right)_{T-t}^{T}(\omega)-\Gamma\left(M^{n_{k}}\right)_{T-t}^{T}(\omega) \\
& =-\left(M^{n_{k}}\right)_{T-t}^{T}(\omega)-2 \Gamma\left(M^{n_{k}}\right)_{T-t}^{T}(\omega),
\end{aligned}
$$

which means that $r_{T} \omega \in \Xi_{T}, M_{t}\left(r_{T} \omega\right)=-M_{T-t}^{T}(\omega)-2 \Gamma(M)_{T-t}^{T}(\omega)$, and $\Gamma(M)_{t}\left(r_{T} \omega\right)=\Gamma(M)_{T-t}^{T}(\omega)$.

Therefore, we obtain the formula (5.1) for the case $M \in \stackrel{\circ}{\mathcal{M}}^{c}$ and $f=$ $\mathbf{1}_{E}$. Replacing $M \in \stackrel{\circ}{\mathcal{M}}^{c}$ with $f * M \in \stackrel{\circ}{\mathcal{M}}^{c}, f \in L^{2}\left(E ; \mu_{\langle M\rangle}\right)$, we obtain the formula (5.1) for all $t \in[0, T]$.

(Step 3): Proof of Theorem 5.2 with (5.1) for $M \in \stackrel{\circ}{\mathcal{M}}$ and $f \in \mathcal{F}_{e} \cap$ $L^{2}\left(E ; \mu_{\langle M\rangle}\right)$.

In this case, we have $M=M^{c}+M^{d}$, and we employ the $r_{T}$-reversible defining set $\Xi_{T}(f * M):=\Xi_{T}\left(f * M^{d}\right) \cap \Xi_{T}\left(f * M^{c}\right)$. We easily get (5.1) for all $t \in[0, T]$.

(Step 4): Finally, we prove Theorems 5.1 and 5.2 for the general case. 
Proof of Theorem 5.1. Take a $Y \in \mathcal{M}_{\text {loc }}^{\llbracket 0, \zeta \llbracket}$ and its corresponding jump function $\varphi \in \mathcal{J}$. Let $\left\{G_{n}\right\}$ be a nest of q.e. finely open Borel sets satisfying $\mathbf{1}_{G_{n} \times E} \varphi \in \stackrel{\circ}{\mathcal{J}}$, and set $Y_{t}^{(n)}:=\mathbf{1}_{G_{n}} * Y \in \stackrel{\circ}{\mathcal{M}}$. Recall that $\mathbf{P}_{x}\left(\lim _{n \rightarrow \infty} \sigma_{E \backslash G_{n}} \geq\right.$ $\zeta)=\mathbf{P}_{x}\left(\lim _{n \rightarrow \infty} \widehat{\sigma}_{E \backslash G_{n}} \geq \zeta\right)=1$ holds for $x \in E \backslash N_{0}$ for some properly exceptional set $N_{0}$ in view of [8, Theorem A.2.3].

Take the reversible defining sets $\left(\Xi_{t}\left(Y^{(n)}\right)\right)$ and $\Xi\left(Y^{(n)}\right)=\bigcap_{t>0} \Xi_{t}\left(Y^{(n)}\right)$ of $Y^{(n)}$. Note that those are still defining sets for $\Gamma\left(Y^{(n)}\right)$ as shown in Steps $1-3$; that is, $\Gamma\left(Y^{(n)}\right)$ is a PrAFS with defining sets $\Xi_{t}\left(Y^{(n)}\right), \Xi\left(Y^{(n)}\right)$.

Let $N_{n}$ be the exceptional set for $\Xi\left(Y^{(n)}\right)$. Then for $n<\ell$ there exists an exceptional set $N_{n, \ell}$ such that $Y_{t}^{(n)}=Y_{t}^{(\ell)}$ for all $t<\tau_{G_{n}} \mathbf{P}_{x^{-}}$-a.s. for $x \in E \backslash N_{n, \ell}$. Choose a properly exceptional set $N$ containing all $N_{n}, n \in$ $\mathbb{N} \cup\{0\}$, and $N_{n, \ell}(1 \leq n<\ell)$. We set

$$
\begin{aligned}
\Xi_{T}(Y):= & \left\{\omega \in \Omega_{T} \cap \bigcap_{n=1}^{\infty} \Xi_{T}\left(Y^{(n)}\right) \mid \text { there exists } n \in \mathbb{N}\right. \text { such that } \\
& T<\sigma_{E \backslash G_{n}}(\omega) \wedge \widehat{\sigma}_{E \backslash G_{n}}(\omega), \\
& \left.Y_{t}^{(n)}(\omega)=Y_{t}^{(\ell)}(\omega) \text { holds for all } t \in[0, T] \text { and } \ell>n\right\},
\end{aligned}
$$

where $\Omega_{T}$ is the set for $N$ as in (6.1). Note that, for $\omega \in \Xi_{T}(Y)$ with $T<$ $\zeta(\omega)$

$$
\begin{aligned}
Y_{t}^{(n)}\left(r_{T} \omega\right) & =-\left(Y^{(n)}\right)_{(T-t)-}^{T-}(\omega)-2\left(\Gamma\left(Y^{(n)}\right)\right)_{T-t}^{T}(\omega), \\
\Gamma\left(Y^{(n)}\right)_{t}\left(r_{T} \omega\right) & =\Gamma\left(Y^{(n)}\right)_{T-t}^{T}(\omega) .
\end{aligned}
$$

For $\omega \in \Xi_{T}(Y)$ with $T<\zeta(\omega)$, there is an $n$ with $T<\tau_{G_{n}}(\omega)$, and set $Y_{t}(\omega):=Y_{t}^{(n)}(\omega)$ for all $t \in[0, T]$. Next we prove the $r_{T}$-reversibility of $\Xi_{T}(Y)$. First note that, for $\omega \in \Xi_{T}(Y), T<\sigma_{E \backslash G_{n}}(\omega)$ (resp., $T<\widehat{\sigma}_{E \backslash G_{n}}(\omega)$ ) implies that $T<\widehat{\sigma}_{E \backslash G_{n}}\left(r_{T} \omega\right)$ (resp., $T<\sigma_{E \backslash G_{n}}\left(r_{T} \omega\right)$ ). Then, we see that $\Xi_{T}(Y)$ is the reversible defining set for $Y$.

Finally, we prove the $\mathscr{F}_{t}^{*}$-measurability of $Y_{t} \circ r_{t}$. Take $C \in \mathscr{B}(\mathbb{R})$. Since $\zeta\left(r_{t} \omega\right)=\infty$ for $t<\zeta(\omega)$,

$$
\begin{aligned}
& \left(Y_{t} \circ r_{t}\right)^{-1}(C) \cap \Xi_{t}(Y) \cap\{t<\zeta\} \\
& \quad=\bigcup_{n=1}^{\infty}\left(Y_{t} \circ r_{t}\right)^{-1}(C) \cap \Xi_{t}(Y) \cap\{t<\zeta\} \cap\left\{t<\tau_{G_{n}} \circ r_{t}\right\}
\end{aligned}
$$




$$
\begin{aligned}
& =\bigcup_{n=1}^{\infty}\left(Y_{t}^{(n)} \circ r_{t}\right)^{-1}(C) \cap \Xi_{t}(Y) \cap\{t<\zeta\} \cap\left\{t<\tau_{G_{n}} \circ r_{t}\right\} \\
& =\bigcup_{n=1}^{\infty}\left(Y_{t}^{(n)} \circ r_{t}\right)^{-1}(C) \cap \Xi_{t}(Y) \cap\{t<\zeta\} \cap\left\{t<\widehat{\sigma}_{E \backslash G_{n}}\right\} \in \mathscr{F}_{t}^{*} .
\end{aligned}
$$

As noted before, we have $\left(Y_{t} \circ r_{t}\right)^{-1}(C) \cap \Xi_{t}(Y) \cap\{t \geq \zeta\}=\emptyset$, or $=\Omega$ and any subset of $\Xi_{t}(Y)^{c}$ is $\mathscr{F}_{t}^{*}$-measurable. Therefore, we obtain the $\mathscr{F}_{t}^{*}$ measurability of $Y_{t} \circ r_{t}$.

Proof of Theorem 5.2 with (5.1). Take an $M \in\left(\mathcal{M}_{\mathrm{loc}}^{\llbracket 0, \zeta \llbracket}\right)_{*}$, and take $f \in$ $\dot{\mathcal{F}}_{\text {loc }}$. Let $\left\{G_{n}\right\}$ be a nest of q.e. finely open Borel sets satisfying $\mathbf{1}_{G_{n} \times E} \varphi \in \stackrel{\circ}{\mathcal{J}}$, $\mathbf{1}_{E \times G_{n}} \varphi \in \stackrel{\circ}{\mathcal{J}}$ and $M_{t}^{(n)}:=\mathbf{1}_{G_{n}} * M \in \stackrel{\circ}{\mathcal{M}}$, and take $\left\{f_{n}\right\} \subset \mathcal{F}_{b}$ such that, for each $n \in \mathbb{N}, f=f_{n}$ q.e. on $G_{n}$. Recall that $\bar{M}^{j}:=-K-M^{j}$. We see that $\mathbf{1}_{G_{n}} * \bar{M}^{j} \in \stackrel{\circ}{\mathcal{M}}$ for each $n \in \mathbb{N}$. Let $K^{(n)} \in \stackrel{\circ}{\mathcal{M}}$ be the purely discontinuous locally square integrable MAF whose jump function is given by $-\mathbf{1}_{G_{n} \times E}(\varphi+$ $\bar{\varphi})$. We then see that $K_{t}=K_{t}^{(n)} t<\tau_{G_{n}} \mathbf{P}_{x^{-}}$a.s. for q.e. $x \in E$.

Take the $r_{T}$-reversible defining set $\Xi_{T}\left(f_{n} * M^{(n)}\right) \cap \Xi_{T}\left(f_{n} * K^{(n)}\right)$ and the defining set $\Xi\left(f_{n} * M^{(n)}\right) \cap \Xi\left(f_{n} * K^{(n)}\right)$ of $f_{n} *\left(M^{(n)}+K^{(n)}\right)$. Note that $\Xi_{T}\left(f_{n} *\left(M^{(n)}+K^{(n)}\right)\right):=\Xi_{T}\left(f_{n} * M^{(n)}\right) \cap \Xi_{T}\left(f_{n} * K^{(n)}\right)$ and $\Xi\left(f_{n} *\right.$ $\left.\left(M^{(n)}+K^{(n)}\right)\right):=\Xi\left(f_{n} * M^{(n)}\right) \cap \Xi\left(f_{n} * K^{(n)}\right)$ are still common defining sets for $f_{n} * \Gamma\left(M^{(n)}\right), f_{n} * \Gamma\left(K^{(n)}\right)$, and $\left[M^{f_{n}}, M^{(n)}+K^{(n)}\right]$.

Let $N_{n}$ be the exceptional set for $\Xi\left(f_{n} * M^{(n)}\right) \cap \Xi\left(f_{n} * K^{(n)}\right)$. Then for $n<\ell$ there exists an exceptional set $N_{n, \ell}$ such that $\left(f_{n} *\left(M^{(n)}+K^{(n)}\right)\right)_{t}=$ $\left(f_{\ell} *\left(M^{(\ell)}+K^{(\ell)}\right)\right)_{t}, f_{n} * \Gamma\left(M^{(n)}\right)_{t}=f_{\ell} * \Gamma\left(M^{(\ell)}\right)_{t}$, and $\left[M^{f_{n}}, M^{(n)}+K^{(n)}\right]_{t}=$ $\left[M^{f_{\ell}}, M^{(\ell)}+K^{(\ell)}\right]_{t}$ for all $t<\tau_{G_{n}} \mathbf{P}_{x^{-}}$a.s. for $x \in E \backslash N_{n, \ell}$. Choose a properly exceptional set $N$ containing all $N_{n}, n \in \mathbb{N}$ and $N_{n, \ell}, n<\ell$. We set

$$
\begin{aligned}
\Xi_{T}(f * M):= & \left\{\omega \in \Omega_{T} \cap \bigcap_{n=1}^{\infty} \Xi_{T}\left(f_{n} *\left(M^{(n)}+K^{(n)}\right)\right) \mid\right. \\
& \text { there exists } n \in \mathbb{N} \text { such that } T<\sigma_{E \backslash G_{n}}(\omega) \wedge \widehat{\sigma}_{E \backslash G_{n}}(\omega), \text { and } \\
& \left(f_{n} *\left(M^{(n)}+K^{(n)}\right)\right)_{t}(\omega)=\left(f_{\ell} *\left(M^{(\ell)}+K^{(\ell)}\right)\right)_{t}(\omega), \\
& \left(f_{n} * \Gamma\left(M^{(n)}\right)\right)_{T-t}^{T}(\omega)=\left(f_{\ell} * \Gamma\left(M^{(\ell)}\right)\right)_{T-t}^{T}(\omega) \text { and } \\
& {\left[M^{f_{n}}, M^{(n)}+K^{(n)}\right]_{t}(\omega)=\left[M^{f_{\ell}}, M^{(\ell)}+K^{(\ell)}\right]_{t}(\omega) } \\
& \text { hold for all } t \in[0, T] \text { and } \ell>n\}
\end{aligned}
$$


where $\Omega_{T}$ is the set for $N$ as in (6.1). Note that, for $\omega \in \Xi_{T}(f * M)$ with $T<\zeta(\omega)$,

$$
\left\{\begin{aligned}
&\left(f_{n} * M^{(n)}\right)_{t}\left(r_{T} \omega\right)=-\left(f_{n} *\left(M^{(n)}+K^{(n)}\right)\right)_{(T-t)-}^{T-}(\omega) \\
&- {\left[M^{f_{n}}, M^{(n)}+K^{(n)}\right]_{(T-t)-}^{T-}(\omega) } \\
&-2\left(f * \Gamma\left(M^{(n)}\right)\right)_{T-t}^{T}(\omega) \\
& {\left[M^{f_{n}}, M^{(n)}+K^{(n)}\right]_{t}\left(r_{T} \omega\right)=-\left[M^{f_{n}}, M^{(n)}+K^{(n)}\right]_{(T-t)-}^{T-}(\omega) } \\
& f * \Gamma\left(M^{(n)}\right)_{t}\left(r_{T} \omega\right)=f * \Gamma\left(M^{(n)}\right)_{T-t}^{T}(\omega)
\end{aligned}\right.
$$

For $\omega \in \Xi_{T}(f * M)$ with $T<\zeta(\omega)$, there is an $n$ with $T<\tau_{G_{n}}(\omega)$, and set $(f * M)_{t}(\omega):=\left(f_{n} * M^{(n)}\right)_{t}(\omega),(f * K)_{t}(\omega):=\left(f_{n} * K^{(n)}\right)_{t}(\omega),\left[M^{f}, M+\right.$ $K]_{t}(\omega):=\left[M^{f_{n}}, M^{(n)}+K^{(n)}\right]_{t}(\omega)$, and $(f * \Gamma(M))_{t}(\omega):=\left(f_{n} * \Gamma\left(M^{(n)}\right)\right)_{t}(\omega)$ for all $t \in[0, T]$. Then from (6.3) we see that $\Xi_{T}(f * M)$ is the reversible defining set for $f * M$. We obtain the desired formula (5.1) for all $t \in[0, T]$. The proof of $\mathscr{F}_{t}^{*}$-measurability of $A_{t} \circ r_{t}$ for each $A=f * M, f * \Gamma(M)$, $f * \Gamma(K),[f(X), M+K]$ is similar to the proof of Theorem 5.1.

Proof of (5.2). It suffices to substitute $K$ for $M$. Recall that $K \in$ $\left(\mathcal{M}_{\mathrm{loc}}^{\llbracket 0, \zeta \llbracket}\right)_{*}, \Gamma(K)=0$, and $\bar{M}^{j}:=-K-M^{j}$. Put $\widetilde{M}^{j}:=\frac{1}{2}\left(M^{j}+\bar{M}^{j}\right)$. Then $K=-2 \widetilde{M^{j}}$. Since the jump function of $K$ is symmetric on $E \times E$, we have $\widetilde{K}^{j}=K$, which implies that the purely discontinuous locally square integrable MAF $\widehat{K}$ from $K$, which is analogously defined like $K$ obtained from $M$, is given by $-2 K$. Hence, $K+\widehat{K}=-K$. Therefore, we obtain (5.2).

\section{§7. Application: Boundary value problem for infinitesimal gener- ator of perturbed semigroup}

A smooth measure $\mu$ is said to be of Kato class if its associated PCAF $A$ satisfies $\| \mathbf{E}$. $\left[A_{t}\right] \|_{\infty} \rightarrow 0$ as $t \rightarrow 0$. Denote by $\mathbf{K}(\mathbf{X})$ the family of all smooth measures of Kato class, and set $\mathbf{K}_{0}(\mathbf{X}):=\{\mu \in \mathbf{K}(\mathbf{X}) \mid \mu(E)<\infty\}$. Let $M$ and $\widehat{M}$ be two locally square integrable local MAFs on $\llbracket 0, \zeta \llbracket$, that is, $M, \widehat{M} \in$

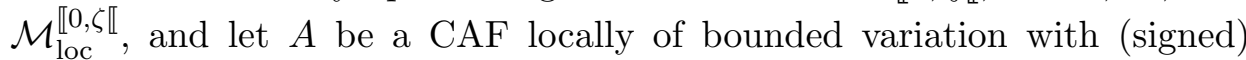
Revuz measure $\mu$. More precisely, $A$ has a representation $A=A^{1}-A^{2}$, where $A^{1}$ and $A^{2}$ are PCAFs with Revuz measures $\mu^{1}$ and $\mu^{2}$, respectively. We set $\mu:=\mu^{1}-\mu^{2}$. Then the signed smooth measure $\mu$ has a Jordan-Hahn decomposition $\mu=\mu^{+}-\mu^{-}$, and set $|\mu|:=\mu^{+}+\mu^{-}$. Similarly to [3, proof of Theorem 3.1], there is a nest $\left\{F_{k}\right\}$ such that $\mathbf{1}_{F_{k}}\left(\mu_{\langle M\rangle}+\mu_{\langle\widehat{M}\rangle}+|\mu|\right) \in \mathbf{K}_{0}(\mathbf{X})$ for all $n$. 
Our main results in this section concern the form perturbation $\mathcal{Q}$ of $(\mathcal{E}, \mathcal{F})$ defined on $\bigcup_{k} \mathcal{F}_{F_{k}}$ by

$$
\begin{aligned}
\mathcal{Q}(f, g)= & \mathcal{E}(f, g)-\int_{E} f(x) \mu_{\left\langle M^{g}, \widehat{M}\right\rangle}(\mathrm{d} x) \\
& -\int_{E} g(x) \mu_{\left\langle M^{f}, M\right\rangle}(\mathrm{d} x)-\int_{E} f(x) g(x) \mu(\mathrm{d} x) \\
& -\int_{E \times E} f(y) g(x) \varphi(x, y) \psi(y, x) N(x, \mathrm{~d} y) \mu_{H}(\mathrm{~d} x) .
\end{aligned}
$$

Here $\varphi \in \mathcal{J}$ and $\psi \in \mathcal{J}$ are Borel functions defined on $E_{\partial} \times E_{\partial}$, vanishing on the diagonal and on $(E \times\{\partial\}) \cup(\{\partial\} \times E)$, and $\varphi, \psi>-1$ on $E \times E$; these are the "jump functions" associated with $M$ and $\widehat{M}$ :

$$
\left.M_{t}-M_{t-}=\varphi\left(X_{t-}, X_{t}\right), \quad \widehat{M}_{t}-\widehat{M}_{t-}=\psi\left(X_{t-}, X_{t}\right) \quad \text { for every } t \in\right] 0, \zeta[,
$$

$\mathbf{P}_{x^{-}}$a.s. for q.e. $x \in E$ by [14, Theorem 1.1] (see [13, Theorem 2.1]).

Now define, for $0 \leq t<\zeta$,

$$
Z_{t}=\operatorname{Exp}\left(M_{t}+A_{t}^{\mu}+\left\langle M^{c}, \widehat{M}^{c}\right\rangle_{t}\right) \cdot \operatorname{Exp}\left(\widehat{M}_{t}\right) \circ r_{t} \cdot\left(1+\psi\left(X_{t}, X_{t-}\right)\right)
$$

wherein Exp denotes the familiar Doléans-Dade stochastic exponential: if $Y$ is a semimartingale on $\llbracket 0, \zeta \llbracket$ with $Y_{0}=0$, then $L=\operatorname{Exp}(Y)$ is the unique solution of the stochastic differential equation

$$
L_{t}=1+\int_{0}^{t} L_{s-} \mathrm{d} Y_{s}, \quad t<\zeta
$$

and is given explicitly by the formula

$$
\operatorname{Exp}\left(Y_{t}\right)=\exp \left(Y_{t}-\frac{1}{2}\left\langle Y^{c}, Y^{c}\right\rangle_{t}\right) \prod_{s \in] 0, t]}\left(1+\Delta Y_{s}\right) e^{-\Delta Y_{s}}, \quad t \in[0, \zeta[.
$$

Now, we define

$$
Q_{t} f(x):=\mathbf{E}_{x}\left[Z_{t} f\left(X_{t}\right)\right]
$$

The statement of Theorem 5.1 yields the following.

LEMma 7.1. Let $Y \in \mathcal{M}_{\mathrm{loc}}^{\llbracket 0, \zeta \llbracket}$ be a locally square integrable $M A F$ on $\llbracket 0, \zeta \llbracket$, and let $\phi \in \mathcal{J}$ be its corresponding jump function. Suppose that $\phi$ is bounded above and that $\phi>-1$ on $E \times E$. Then $L_{t}:=\operatorname{Exp}\left(Y_{t}\right)$ is an rcll-PrMFS on $\llbracket 0, \zeta \llbracket$, and $L_{t} \circ r_{t}\left(1+\phi\left(X_{t}, X_{t-}\right)\right)$ is an rcll-PrMF* on $\llbracket 0, \zeta \llbracket$. 
Corollary 7.1. The functional $Z_{t}$ is a $\operatorname{Pr} M F^{*}$ on $\llbracket 0, \zeta \llbracket$. In particular, $Z_{t+s}=Z_{t}\left(Z_{s} \circ \theta_{t}\right)$ holds for all $s, t>0$ with $t+s<\zeta \mathbf{P}_{x}$-a.s. for q.e. $x \in E$.

REMARK 7.1. In [4] we deduce only that $Z_{t}$ is an rcll-PrMF admitting an m-null set, which is derived from [4, Theorem 2.18]. The content of Corollary 7.1 is a refinement of this observation.

Proof of Lemma \%.1. It suffices to prove the case for $-1+\varepsilon \leq \phi \leq 1 / \varepsilon$ on $E \times E$ for some $\varepsilon \in] 0,1[$. Note that there exists $C(\varepsilon) \in[1 / 2, \infty[$ such that $|x-\log (1+x)| \leq C(\varepsilon) x^{2}$ for $x \in[-1+\varepsilon, 1 / \varepsilon]$. Then we see that $N(\mid \phi-$ $\log (1+\phi) \mid) \mu_{H} \in S$ and that $N\left((\phi-\log (1+\phi))^{2}\right) \mu_{H} \in S$. In particular,

$$
\begin{aligned}
M_{t}^{(\phi)}:= & \sum_{0<s \leq t}\left\{\phi\left(X_{s-}, X_{s}\right)-\log \left(1+\phi\left(X_{s-}, X_{s}\right)\right)\right\} \\
& -\int_{0}^{t} N(\phi-\log (1+\phi))\left(X_{s}\right) \mathrm{d} H_{s}
\end{aligned}
$$

is a locally square integrable MAF on $\llbracket 0, \zeta \llbracket$. Let $A_{t}:=\log L_{t}$. Theorem 5.1 and Corollary 6.1 together imply that

$$
A_{t}=Y_{t}-\frac{1}{2}\left\langle Y^{c}\right\rangle_{t}-M_{t}^{(\phi)}-\int_{0}^{t} N(\phi-\log (1+\phi))\left(X_{s}\right) \mathrm{d} H_{s}
$$

can be regarded as an rcll reversible PrAFS with $A_{t}-A_{t-}=\log \left(1+\phi\left(X_{t-}\right.\right.$, $\left.X_{t}\right)$ ) for all $t>0 \mathbf{P}_{x^{-}}$a.s. for q.e. $x \in E$. Applying Lemma 4.3 to $A, \widehat{A}_{t}=$ $A_{t} \circ r_{t}+\log \left(1+\phi\left(X_{t}, X_{t-}\right)\right)$ is an rcll-PrAF* on $\llbracket 0, \zeta \llbracket ;$ hence, $L_{t} \circ r_{t}(1+$ $\left.\phi\left(X_{t}, X_{t-}\right)\right)=\exp \widehat{A}_{t}$ is an rcll-PrMF* on $\llbracket 0, \zeta \llbracket$.

Proof of Corollary 7.1. The assertion follows from Lemma 7.1, provided that $\varphi$ and $\psi$ are bounded above and below away from -1 . Next we prove the assertion for general $\varphi>-1$ and $\psi>-1$. For $\ell \geq 2$, we set $\varphi_{\ell}:=$ $\mathbf{1}_{[-1+1 / \ell, \ell]}(\varphi) \mathbf{1}_{]-1 / \ell, 1 / \ell\left[^{c}\right.}(\varphi) \varphi$ (resp., $\left.\psi_{\ell}:=\mathbf{1}_{[-1+1 / \ell, \ell]}(\psi) \mathbf{1}_{]-1 / \ell, 1 / \ell\left[{ }^{c}\right.}(\psi) \psi\right)$, and let $M^{d, \ell}$ (resp., $\widehat{M}^{d, \ell}$ ) be its corresponding purely discontinuous locally square integrable MAF on $\llbracket 0, \zeta \llbracket$. We can define $Z_{t}^{\ell}$, as well as $Z_{t}$, by replac$\operatorname{ing} M^{d}, \widehat{M}^{d}, \varphi, \psi$ with $M^{d, \ell}, \widehat{M}^{d, \ell}, \varphi_{\ell}, \psi_{\ell}$, respectively. Recall that we can construct a subsequence $\left\{\ell_{k}\right\}$ of $\{\ell\}$ such that $M^{d, \ell_{k}}$ (resp., $\widehat{M}^{d, \ell_{k}}$ ) uniformly converges to $M^{d}$ (resp., $\widehat{M}^{d}$ ) on each compact subinterval of $[0, \zeta[$ $\mathbf{P}_{x^{-}}$a.s. for q.e. $x \in E$. Consequently, $Z^{\ell_{k}}$ uniformly converges to $Z$ on each compact subinterval of $\left[0, \zeta\left[\mathbf{P}_{x}\right.\right.$-a.s. for q.e. $x \in E$. Therefore, the assertion follows from this observation. 
Recall that a signed smooth measure $\nu$ is said to be of Hardy class (denoted by $\nu \in \mathbf{H}(\mathbf{X})$ ) if there are constants $\delta>0$ and $\gamma \geq 0$ such that

$$
\int_{E} u(x)^{2}|\nu|(\mathrm{d} x) \leq \delta \cdot \mathcal{E}(u, u)+\gamma \cdot(u, u)_{\mathfrak{m}} \quad \text { for every } u \in \mathcal{F},
$$

where $|\nu|:=\nu^{+}+\nu^{-}$denotes the total variation measure of $\nu$. Note that every $\nu \in \mathbf{H}(\mathbf{X})$ is a Radon measure. It is known that $\mathbf{K}(\mathbf{X}) \subset \mathbf{H}(\mathbf{X})$, and for $\nu \in \mathbf{K}(\mathbf{X})$ the constant $\delta$ satisfying (7.4) can be taken to be arbitrarily small. Also, we write $\mathcal{F}_{b}$ for the class of (m-essentially) bounded elements of $\mathcal{F}$. For $\mu_{\langle M\rangle}, \mu_{\langle\widehat{M}\rangle},|\mu| \in \mathbf{H}(\mathbf{X})$, there exists $C>0$ depending on $\mu_{\langle M\rangle}, \mu_{\langle\widehat{M}\rangle}, \mu$ such that $|\mathcal{Q}(f, g)| \leq C \mathcal{E}_{1}(f, f)^{1 / 2} \mathcal{E}_{1}(g, g)^{1 / 2}$ for any $f, g \in \mathcal{F}$.

The following theorem is proved in [3].

Theorem 7.1 ([3, Theorem 3.1]). Assume that $\mu_{\langle M\rangle}, \mu_{\langle\widehat{M}\rangle}$ and $|\mu|$ are all in the Hardy class $\mathbf{H}(\mathbf{X})$, and that there are constants $\alpha>0$ and $c>1$ such that

$$
c^{-1} \mathcal{E}_{1}(u, u) \leq \mathcal{Q}_{\alpha}(u, u) \leq c \mathcal{E}_{1}(u, u) \quad \text { for } u \in \mathcal{F}_{b}
$$

Then $Q_{t} f(x), f \in L^{2}(E ; \mathfrak{m}) \cap \mathscr{B}(E)$ defined by (7.6) coincides with the strongly continuous semigroup in $L^{2}(E ; \mathfrak{m})$ associated with $(\mathcal{Q}, \mathcal{F})$.

As a consequence of the above result, the following result is also established in [3, Theorem 3.1] and [5, Theorem 1.7].

THEOREM 7.2 (special case of [5, Theorem 1.7]). Suppose that $M \in$ $\left(\mathcal{M}_{\mathrm{loc}}^{\llbracket 0, \zeta \llbracket}\right)_{*}$ is a locally square integrable local $M A F$ on $\llbracket 0, \zeta \llbracket$ of $\mathbf{X}$ with jump function $\varphi \in \mathcal{J}_{*}$ such that $\mu_{\langle M\rangle} \in \mathbf{H}(\mathbf{X})$. Define

$$
\mathcal{Q}(f, g):=\mathcal{E}(f, g)+\frac{1}{2} \mu_{\left\langle M^{f g}+M^{f g, \kappa}, M\right\rangle}(E) \quad \text { for } f, g \in \mathcal{F}_{b} .
$$

Suppose that there are constants $\alpha>0$ and $c>1$ so that

$$
c^{-1} \mathcal{E}_{1}(u, u) \leq \mathcal{Q}_{\alpha}(u, u) \leq c \mathcal{E}_{1}(u, u) \quad \text { for } u \in \mathcal{F}_{b}
$$

Then $\bar{P}_{t} f(x):=\mathbf{E}_{x}\left[e^{\Lambda(M)_{t}} f\left(X_{t}\right)\right], f \in L^{2}(E ; \mathfrak{m}) \cap \mathscr{B}(E)$ is the symmetric semigroup associated with $(\mathcal{Q}, \mathcal{F})$, where

$$
\Lambda(M)_{t}:=-\frac{1}{2}\left(M_{t}+M_{t} \circ r_{t}+\varphi\left(X_{t}, X_{t-}\right)\right), \quad 0<t<\zeta .
$$


For $u \in \mathcal{F}_{e}, M^{u}$ is an MAF of $\mathbf{X}$ having finite energy with antisymmetric jump function $u(y)-u(x)$ and $\Lambda\left(M^{u}\right)=N^{u}$. The following corollary is an immediate consequence of Theorem 7.2.

Corollary 7.2. Suppose that $u \in \mathcal{F}_{e}$ with $\mu_{\left\langle M^{u}\right\rangle} \in \mathbf{H}(\mathbf{X})$. Define

$$
\mathcal{Q}(f, g):=\mathcal{E}(f, g)+\mathcal{E}(f g, u) \quad \text { for } f, g \in \mathcal{F}_{b} .
$$

Suppose that there are constants $\alpha>0$ and $c>1$ so that

$$
c^{-1} \mathcal{E}_{1}(u, u) \leq \mathcal{Q}_{\alpha}(u, u) \leq c \mathcal{E}_{1}(u, u) \quad \text { for } u \in \mathcal{F}_{b}
$$

Then $\bar{P}_{t} f(x):=\mathbf{E}_{x}\left[e^{N_{t}^{u}} f\left(X_{t}\right)\right], f \in L^{2}(E ; \mathfrak{m}) \cap \mathscr{B}(E)$ is a strongly continuous symmetric semigroup in $L^{2}(E ; \mathfrak{m})$ associated with $(\mathcal{Q}, \mathcal{F})$.

Our first main theorem in this section is the following.

Theorem 7.3. For $f \in L^{2}(E ; \mathfrak{m}) \cap \mathscr{B}(E)$, all semigroups $Q_{t} f$ and $\bar{P}_{t} f$ that appeared in Theorems $\% .1$ and $\% .2$ and Corollary 7.2 are $\mathcal{E}$-quasicontinuous.

To prove Theorem 7.3, we need the following lemma.

Lemma 7.2. Suppose that $\mu_{\langle M\rangle}, \mu_{\langle\widehat{M}\rangle}, \mu \in \mathbf{K}_{0}(\mathbf{X})$ hold. Then we have

$$
\sup _{s \in[0, t]} Z_{s}<\infty \quad \mathbf{P}_{x} \text {-a.s. on }\{t<\zeta\} \text { for q.e. } x \in E \text {. }
$$

Proof. It suffices to show that $\sup _{s \in[0, t]} \operatorname{Exp}\left(M_{s}^{d}\right) \operatorname{Exp}\left(\widehat{M}_{s}^{d}\right) \circ r_{s}\left(1+\psi\left(X_{s-}\right.\right.$, $\left.\left.X_{s}\right)\right)<\infty \mathbf{P}_{x^{-}}$a.s. for q.e. $x \in E$. First, we assume that $|\varphi|$ and $|\psi|$ are bounded below by $\varepsilon>0$. Under this lower bound, we have $M, \widehat{M} \in \stackrel{\circ}{\mathcal{M}}$ and $N(|\varphi|) \mu_{H}, N(|\psi|) \mu_{H} \in \mathbf{K}_{0}(\mathbf{X})$, because of $\varepsilon|\varphi| \leq \varphi^{2}, \varepsilon|\psi| \leq \psi^{2}$. Moreover, since $\int_{E} N\left(\bar{\psi}^{2}\right) \mathrm{d} \mu_{H}=\int_{E} N\left(\psi^{2}\right) \mathrm{d} \mu_{H}=2 \mathbf{e}(\widehat{M})<\infty$, there exists an MAF $\overline{\widehat{M}}^{d} \in \stackrel{\circ}{\mathcal{M}}$ such that $\Delta{\overline{\widehat{M}_{t}}}_{t}^{d}=\bar{\psi}\left(X_{t-}, X_{t}\right)$ and $\left\langle\overline{\widehat{M}}^{d}\right\rangle_{t}=\int_{0}^{t} N\left(\bar{\psi}^{2}\right)\left(X_{s}\right) \mathrm{d} H_{s}$ for all $t \in\left[0, \infty\left[\mathbf{P}_{x}\right.\right.$-a.s. for q.e. $x \in E$. Since

$$
\operatorname{Exp}\left(M_{t}^{d}\right) \leq \exp \left(M_{t}^{d}\right)=\exp \left(\sum_{s \leq t} \varphi\left(X_{s-}, X_{s}\right)-\int_{0}^{t} N(\varphi)\left(X_{s}\right) \mathrm{d} H_{s}\right)
$$

we have $\sup _{s \in[0, t]} \operatorname{Exp}\left(M_{s}^{d}\right)<\infty \mathbf{P}_{x^{-}}$a.s. for q.e. $x \in E$. Let $\left\{F_{n}\right\}$ be an $\mathcal{E}$-nest such that $\mathbf{1}_{F_{n}} N\left(\bar{\psi}^{2}\right) \mu_{H} \in \mathbf{K}_{0}(\mathbf{X})$ for each $n \in \mathbb{N}$. Similarly, by Theorem 5.2, the following holds $\mathbf{P}_{x}$-a.s. on for $\{t<\zeta\}$ for q.e. $x \in E$ :

$$
\operatorname{Exp}\left(\widehat{M}_{t}^{d}\right) \circ r_{t}\left(1+\psi\left(X_{t}, X_{t-}\right)\right)
$$




$$
\begin{aligned}
& \leq \exp \left(\widehat{M}_{t}^{d} \circ r_{t}\right)\left(1+\psi\left(X_{t}, X_{t-}\right)\right) \\
& =\exp \left(\widehat{\widehat{M}}_{t-}^{d}-2 \Gamma\left(\widehat{M}^{d}\right)_{t}\right)\left(1+\psi\left(X_{t}, X_{t-}\right)\right) \\
& \leq \exp \left(-2 \Gamma\left(\widehat{M}^{d}\right)_{t}+\sum_{s \leq t} \bar{\psi}\left(X_{s-}, X_{s}\right)-\int_{0}^{t} N(\bar{\psi})\left(X_{s}\right) \mathrm{d} H_{s}\right)
\end{aligned}
$$

Then we see that $\sup _{s \in[0, t]} \operatorname{Exp}\left(\widehat{M}_{s}^{d}\right) \circ r_{s}\left(1+\psi\left(X_{s}, X_{s-}\right)\right)<\infty \mathbf{P}_{x^{-}}$a.s. on $\left\{t<\tau_{F_{n}}\right\}$ for q.e. $x \in E$ for each $n \in \mathbb{N}$, because of $\mathbf{1}_{F_{n}} N(|\bar{\psi}|) \mu_{H} \in \mathbf{K}_{0}(\mathbf{X})$. Consequently, we have

$\sup _{s \in[0, t]} \operatorname{Exp}\left(\widehat{M}_{s}^{d}\right) \circ r_{s}\left(1+\psi\left(X_{s}, X_{s-}\right)\right)<\infty \quad \mathbf{P}_{x^{-}}$a.s. on $\{t<\zeta\}$ for q.e. $x \in E$. $s \in[0, t]$

Finally, we prove the assertion without the boundedness for $\varphi, \psi$. Set $\varphi_{\ell}:=\mathbf{1}_{-1 / \ell, 1 / \ell\left[^{c}\right.}(\varphi) \varphi$, and set $\psi_{\ell}:=\mathbf{1}_{]-1 / \ell, 1 / \ell\left[^{c}\right.}(\psi) \psi$. Let $M^{(\ell)} \in \dot{\mathcal{M}}$ (resp. $\left.\widehat{M}^{(\ell)} \in \stackrel{\circ}{\mathcal{M}}\right)$ be an MAF of finite energy whose continuous part and killing part coincide with $M^{c}, M^{\kappa}$ (resp., $\left.\widehat{M}^{c}, \widehat{M}^{\kappa}\right)$, respectively, and its jump

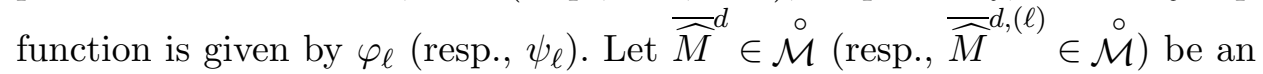
MAF of finite energy whose continuous part and killing part coincide with $\widehat{M}^{c}, \widehat{M}^{\kappa}$ and its jump function is given by $\bar{\psi}$ (resp., $\left.\bar{\psi}_{\ell}\right)$. Note that $\mathbf{e}\left(M^{(\ell)}-\right.$ $M)=\frac{1}{2} \int_{E} N\left(\left(\varphi_{\ell}-\varphi\right)^{2}\right)(x) \mu_{H}(\mathrm{~d} x) \rightarrow 0$, that $\mathbf{e}\left(\widehat{M}^{(\ell)}-\widehat{M}\right)=\frac{1}{2} \int_{E} N\left(\left(\psi_{\ell}-\right.\right.$ $\left.\psi)^{2}\right)(x) \mu_{H}(\mathrm{~d} x)=\frac{1}{2} \int_{E} N\left(\left(\bar{\psi}_{\ell}-\bar{\psi}\right)^{2}\right)(x) \mu_{H}(\mathrm{~d} x)=\mathbf{e}\left(\widehat{\widehat{M}}^{d,(\ell)}-\widehat{\widehat{M}}^{d}\right) \rightarrow 0$ as $\ell \rightarrow$ $\infty$, and that there exists a subsequence $\left\{\ell_{k}\right\}$ such that $M^{\left(\ell_{k}\right)} \rightarrow M, \widehat{M}^{\left(\ell_{k}\right)} \rightarrow$ $\widehat{M}, \widehat{\widehat{M}}^{d,(\ell)} \rightarrow \widehat{\widehat{M}}^{d}$ uniformly converge on each compact interval $\mathbf{P}_{x^{-}}$-a.s. for q.e. $x \in E$. Moreover, by [18, Theorem 3.2], $\Gamma\left(\widehat{M}^{\left(\ell_{k}\right)}\right) \rightarrow \Gamma(\widehat{M})$ uniformly converges on each compact interval $\mathbf{P}_{x}$-a.s. for q.e. $x \in E$. These convergences imply that $\sup _{s \in[0, t]} \operatorname{Exp}\left(M_{s}\right)<\infty \mathbf{P}_{x}$-a.s. for q.e. $x \in E$ and that $\sup _{s \in[0, t]} \operatorname{Exp}\left(\widehat{M}_{s}\right) \circ r_{s}\left(1+\psi\left(X_{s}, X_{s-}\right)\right)<\infty \mathbf{P}_{x^{-}}$a.s. on $\{t<\zeta\}$ for q.e. $x \in$ $E$. Therefore, we obtain the assertion.

Proof of Theorem 7.3. The proof of the $\mathcal{E}$-quasicontinuity of $\bar{P}_{t} f$ is similar to the proof of that for $Q_{t} f$, so we prove only for the case $Q_{t} f$.

(Step 1): We first assume that $\mu_{\langle M\rangle}, \mu_{\langle\widehat{M}\rangle}, \mu \in \mathbf{K}_{0}(\mathbf{X})$.

Set $S_{\alpha} f(x):=\mathbf{E}_{x}\left[\int_{0}^{\infty} e^{-\alpha t} Z_{t} f\left(X_{t}\right) \mathrm{d} t\right]$ for $\alpha>0$ and $f \in L^{2}(E ; \mathfrak{m}) \cap \mathscr{B}(E)$, and take $\alpha_{0}>0$ with $\mathcal{Q}_{\alpha_{0}}(u, u) \geq c^{-1} \mathcal{E}_{1}(u, u)$ for $u \in \mathcal{F}$. From Theorem 7.1 ([3, Theorem 3.1]), we know that $S_{\alpha} f \in \mathcal{F}$ and $\mathcal{Q}_{\alpha}\left(S_{\alpha} f, g\right)=(f, g)_{\mathfrak{m}}$ for 
$\alpha>\alpha_{0}$ and $g \in \mathcal{F}$. We first prove the $\mathcal{E}$-quasicontinuity of $S_{\alpha} f$ under the condition $f \in L^{2}(E ; \mathfrak{m}) \cap \mathscr{B}_{b}(E)$ and $\alpha>\alpha_{0}$. By Corollary 7.1, for nonnegative $f \in L^{2}(E ; \mathfrak{m}) \cap \mathscr{B}_{b}(E)$, the following holds under $\mathbf{P}_{x}$ for q.e. $x \in E$ :

$$
\begin{aligned}
e^{-\alpha t} Z_{t} S_{\alpha} f\left(X_{t}\right) & =\mathbf{E}_{X_{t}}\left[\int_{0}^{\infty} e^{-\alpha(t+s)} Z_{t} Z_{s} f\left(X_{s}\right) \mathrm{d} s\right] \\
& =\mathbf{E}_{x}\left[\int_{0}^{\infty} e^{-\alpha(t+s)} Z_{t}\left(Z_{s} \circ \theta_{t}\right) f\left(X_{t+s}\right) \mathrm{d} s \mid \mathscr{F}_{t}\right] \\
& =\mathbf{E}_{x}\left[\int_{t}^{\infty} e^{-\alpha s} Z_{s} f\left(X_{s}\right) \mathrm{d} s \mid \mathscr{F}_{t}\right] \\
& =\mathbf{E}_{x}\left[\int_{0}^{\infty} e^{-\alpha s} Z_{s} f\left(X_{s}\right) \mathrm{d} s \mid \mathscr{F}_{t}\right]-\int_{0}^{t} e^{-\alpha s} Z_{s} f\left(X_{s}\right) \mathrm{d} s .
\end{aligned}
$$

Owing to Lemma 7.2, the second term of the right-hand side is finite $\mathbf{P}_{x^{-}}$ a.s. on $\{t<\zeta\}$ for q.e. $x \in E$; hence, the right-hand side is right-continuous at $t \in\left[0, \zeta\left[\right.\right.$ under $\mathbf{P}_{x}$ for q.e. $x \in E$. Consequently, $S_{\alpha} f\left(X_{t}\right)$ is so at all $t \in\left[0, \infty\left[\right.\right.$, provided that $\alpha>\alpha_{0}$.

The right continuity of $t \mapsto S_{\alpha} f\left(X_{t}\right)$ under $\mathbf{P}_{x}$ for q.e. $x \in E$ implies that $S_{\alpha} f$ is $[0, \infty]$-valued q.e. finely continuous; hence, it is $[0, \infty]$-valued $\mathcal{E}$-quasicontinuous for $\alpha>\alpha_{0}$. Since $S_{\alpha} f$ has an $\mathcal{E}$-quasicontinuous $\mathfrak{m}$-version $\widetilde{S_{\alpha} f}$, we see that $S_{\alpha} f=\widetilde{S_{\alpha} f}$ q.e.; hence, $S_{\alpha} f$ is q.e. finite and $\mathcal{E}$-quasicontinuous.

Next we prove the $\mathcal{E}$-quasicontinuity of $S_{\alpha} f$ for $f \in L^{2}(E ; \mathfrak{m}) \cap \mathscr{B}(E)$ and $\alpha>\alpha_{0}$. We may assume that $f \geq 0$ on $E$. Set $f_{n}:=f \wedge n$ for $n \in \mathbb{N}$. Then $\left\{S_{\alpha} f_{n}\right\}_{n \in \mathbb{N}}$ is $\mathcal{E}$-bounded. Indeed, for $\alpha>\alpha_{0}$,

$$
\begin{aligned}
c^{-1} \mathcal{E}_{1}\left(S_{\alpha} f_{n}, S_{\alpha} f_{n}\right) & \leq \mathcal{Q}_{\alpha}\left(S_{\alpha} f_{n}, S_{\alpha} f_{n}\right)=\left(f_{n}, S_{\alpha} f_{n}\right)_{\mathfrak{m}} \leq\left\|f_{n}\right\|_{2} \|_{S_{\alpha} f_{n} \|_{2}} \\
& \leq\left\|f_{n}\right\|_{2}^{2} /\left(\alpha-\alpha_{0}\right) \leq\|f\|_{2}^{2} /\left(\alpha-\alpha_{0}\right)
\end{aligned}
$$

because $\left(\alpha-\alpha_{0}\right)\left\|S_{\alpha} f_{n}\right\|_{2}^{2} \leq \mathcal{Q}_{\alpha}\left(S_{\alpha} f_{n}, S_{\alpha} f_{n}\right)=\left(f_{n}, S_{\alpha} f_{n}\right)_{\mathfrak{m}} \leq\left\|f_{n}\right\|_{2}\left\|S_{\alpha} f_{n}\right\|_{2}$. We easily see that $S_{\alpha} f_{n}$ converges to $S_{\alpha} f$ as $n \rightarrow \infty$ q.e. Then Lemma 2.2 yields the $\mathcal{E}$-quasicontinuity of $S_{\alpha} f$ for $\alpha>\alpha_{0}$.

(Step 2): Finally, we prove the assertion for the general case.

We can construct an $\mathcal{E}$-nest $\left\{F_{n}\right\}$ of closed sets such that $\mathbf{1}_{F_{n}}\left(\mu_{\langle M\rangle}+\right.$ $\left.\mu_{\langle\widehat{M}\rangle}+|\mu|\right) \in \mathbf{K}_{0}(\mathbf{X})$, and there exists $\rho_{n} \in \mathcal{F}$ such that $\Gamma\left(\mathbf{1}_{F_{n}} \widehat{M}^{c}\right)_{t}=N_{t}^{\rho_{n}}-$ $\int_{0}^{t} \rho_{n}\left(X_{s}\right) \mathrm{d} s$ for $t \in\left[0, \tau_{F_{n}}\left[\mathbf{P}_{x^{-}}\right.\right.$-a.s. for q.e. $x \in E,\left.\rho_{n}\right|_{F_{n}} \in C\left(F_{n}\right)$ and $\mathbf{1}_{F_{n}} \mu_{\left\langle\rho_{n}\right\rangle} \in \mathbf{K}_{0}(\mathbf{X})$ for each $n \in \mathbb{N}$ (see [3, proof of Theorem 3.1]). Let $E_{n}$ 
be the fine interior of $F_{n}$. Then $\mathbf{1}_{E_{n}}\left(\mu_{\langle M\rangle}+\mu_{\langle\widehat{M}\rangle}+|\mu|\right) \in \mathbf{K}_{0}\left(\mathbf{X}^{E_{n}}\right)$. The conclusion of [3, proof of Theorem 3.1] tells us that, for all $\alpha>\alpha_{0}, S_{\alpha}^{(n)} f$ defined by $S_{\alpha}^{(n)} f(x):=\mathbf{E}_{x}\left[\int_{0}^{\tau_{E_{n}}} e^{-\alpha t} Z_{t} f\left(X_{t}\right)\right]$ for $f \in L^{2}(E ; \mathfrak{m}) \cap \mathscr{B}(E)$ satisfies $S_{\alpha}^{(n)} f \in \mathcal{F}_{E_{n}}$ and $\mathcal{Q}_{\alpha}\left(S_{\alpha}^{(n)} f, g\right)=(f, g)_{\mathfrak{m}}$ for $g \in \mathcal{F}_{E_{n}}$. By Step $1, S_{\alpha}^{(n)} f$ is $\mathcal{E}_{E_{n}}$-quasicontinuous on $E_{n}$, hence $\mathcal{E}$-quasicontinuous on $E_{n}$ for each $n \in \mathbb{N}$; consequently, it is $\mathcal{E}$-quasicontinuous on $E_{k}$ for all $n>k$ in view of Lemma 2.1.

Noting the $\mathcal{E}$-boundedness of $S_{\alpha}^{(n)} f$ with respect to $n$ for each $f \in L^{2}(E$; $\mathfrak{m}) \cap \mathscr{B}(E)$, Lemma 2.2 implies the $\mathcal{E}$-quasicontinuity of $S_{\alpha} f, f \in L^{2}(E ; \mathfrak{m}) \cap$ $\mathscr{B}(E)$. The proof of the $\mathcal{E}$-quasicontinuity of $Q_{t} f$ for $f \in L^{2}(E ; \mathfrak{m}) \cap \mathscr{B}(E)$ is similar to [8, proof of Theorem 4.2.3(ii)], so we omit it.

Finally, we show the second main theorem of this section. From now on, we assume that $\mathbf{X}$ is a transient strong Feller process, $\mu_{\langle M\rangle}, \mu_{\langle\widehat{M}\rangle},|\mu| \in$ $\mathbf{K}_{\infty}(\mathbf{X})$, and that $(\mathcal{E}, \mathcal{F})$ is irreducible (see [8, p. 171] for the definition of irreducibility). Here $\mathbf{K}_{\infty}(\mathbf{X}):=\left\{\nu \in \mathbf{K}(\mathbf{X}) \mid \lim _{n \rightarrow \infty}\left\|R \mathbf{1}_{K_{n}^{c}} \nu\right\|_{\infty}=0\right.$ for any increasing sequence of compact sets $\left.\left\{K_{n}\right\}\right\}$ is the family of Green-tight measures of Kato class. It is known that $\nu \in \mathbf{K}_{\infty}(\mathbf{X})$ satisfies $\|R \nu\|_{\infty}<\infty$. We suppose that

$$
\begin{aligned}
& \sqrt{2\left\|R \mu_{\langle M\rangle}\right\|_{\infty}}+\sqrt{2\left\|R \mu_{\langle\widehat{M}\rangle}\right\|_{\infty}}+\|R \mid \mu\|_{\infty} \\
& \quad+2 \sqrt{\left\|R N\left(\varphi^{2}\right) \mu_{H}\right\|_{\infty}} \sqrt{\left\|R N\left(\psi^{2}\right) \mu_{H}\right\|_{\infty}}<1 .
\end{aligned}
$$

Here $R \nu(x):=\mathbf{E}_{x}\left[A_{\zeta}^{\nu}\right]$, where $A^{\nu}$ is a PCAF associated with the smooth measure $\nu$. Then $\mathcal{Q}$ can be extended to $\mathcal{F}_{e} \times \mathcal{F}_{e}$, and there exists $c>1$ such that

$$
\left\{\begin{array}{l}
\mathcal{E}(u, u) \leq c \mathcal{Q}(u, u), \\
|\mathcal{Q}(u, v)| \leq c \mathcal{E}(u, u)^{1 / 2} \mathcal{E}(v, v)^{1 / 2} \quad \text { for any } u, v \in \mathcal{F}_{e} .
\end{array}\right.
$$

In particular, $(\mathcal{Q}, \mathcal{F})$ is a strongly sectorial coercive closed form on $L^{2}(E ; \mathfrak{m})$ with lower bound 0 . If $\Gamma$ is a nonempty closed subspace of $\mathcal{F}$ (resp., $\mathcal{F}_{e}$ for $\alpha=0$ ), then [17, Chapter I, Theorem 2.6] tells us that, for any $u \in \mathcal{F}$ (resp., $u \in \mathcal{F}_{e}$ ) and each $\alpha>0$ (resp., $\alpha=0$ ), there exists unique $\Pi_{\Gamma}^{\alpha} u \in \mathcal{F}$ (resp., $\left.\Pi_{\Gamma}^{0} u \in \mathcal{F}_{e}\right)$ such that $\mathcal{Q}_{\alpha}\left(u-\Pi_{\Gamma}^{\alpha} u, w\right)=0$ (resp., $\mathcal{Q}\left(u-\Pi_{\Gamma}^{0} u, w\right)=0$ ) for any $w \in \Gamma$. Let $D$ be a nonempty open subset of $E$ such that $E \backslash D$ is non- $m-$ polar, and we set $B:=E \backslash D$. For $u \in \mathcal{F}$ (resp., $u \in \mathcal{F}_{e}$ ), we write $u_{B}^{\alpha}:=$ $u-\Pi_{\mathcal{F}_{D}}^{\alpha} u$ (resp., $u_{B}:=u-\Pi_{\left(\mathcal{F}_{D}\right)_{e}}^{0} u$ ) and call it the $\alpha$-reduced function of $u$ 
on $B$. Since $\mathcal{Q}_{\alpha}\left(u_{B}^{\alpha}, u_{B}^{\alpha}\right) \leq 2 \mathcal{Q}_{\alpha}\left(u, \Pi_{\mathcal{F}_{D}}^{\alpha} u\right)+2 \mathcal{Q}_{\alpha}(u, u)$ (resp., $\mathcal{Q}\left(u_{B}, u_{B}\right) \leq$ $\left.2 \mathcal{Q}\left(u, \Pi_{\left(\mathcal{F}_{D}\right) e} u\right)+2 \mathcal{Q}(u, u)\right)$ for $u \in \mathcal{F}\left(\right.$ resp., $\left.u \in \mathcal{F}_{e}\right)$, we have $\mathcal{Q}_{\alpha}\left(u_{B}^{\alpha}, u_{B}^{\alpha}\right) \leq$ $C \mathcal{Q}_{\alpha}(u, u)$, where $C$ is the constant independent of $\alpha \in[0,1]$. In the same way as [17, Chapter III. Proposition 1.5(iii)], $u_{B}^{\alpha}$ is the smallest function $v$ such that $e^{-\alpha t} Q_{t}\left(v \wedge u_{B}^{\alpha}\right) \leq v \wedge u_{B}^{\alpha}$ m-a.e. for all $t>0$ and $v \geq u$ q.e. on $B$.

For q.e.-defined Borel function $u$, we set $Q_{B}^{\alpha} u(x):=\mathbf{E}_{x}\left[e^{-\alpha \tau_{D}} Z_{\tau_{D}} u\left(X_{\tau_{D}}\right)\right.$ : $\left.\tau_{D}<\zeta\right]$ for $\alpha \geq 0$ if its expectation has meaning, and we write $Q_{B} u:=Q_{B}^{0} u$.

Lemma 7.3. For each $u \in \mathcal{F}$ and $\alpha>0, Q_{B}^{\alpha} u$ is an $\mathcal{E}$-quasicontinuous $\mathfrak{m}-$ version of $u_{B}^{\alpha}$. In particular,

$$
\mathcal{Q}_{\alpha}\left(Q_{B}^{\alpha} u, v\right)=0 \quad \text { for any } v \in \mathcal{F}_{D}
$$

Moreover, for $u \in \mathcal{F}_{e}, Q_{B} u$ is an $\mathcal{E}$-quasicontinuous $\mathfrak{m}$-version of $u_{B}$. In particular,

$$
\mathcal{Q}\left(Q_{B} u, v\right)=0 \quad \text { for any } v \in\left(\mathcal{F}_{D}\right)_{e}
$$

Proof. First we assume that $u=S_{\alpha} f \in \mathcal{F}$ for $f \in L^{2}(E ; \mathfrak{m}) \cap \mathscr{B}(E)$ for $\alpha>0$. We may assume that $f \geq 0$ on $E$. From this, we see that $e^{-\alpha t} Q_{t} Q_{B}^{\alpha} u \leq$ $Q_{B}^{\alpha} u \mathfrak{m}$-a.e. for all $t>0$. It suffices to prove that

$$
\begin{aligned}
& Q_{B}^{\alpha} u \leq u_{B}^{\alpha} \quad \text { m-a.e., } \\
& Q_{B}^{\alpha} u=u \quad \text { q.e. on } B .
\end{aligned}
$$

Indeed, under (7.6) we have $e^{-\alpha t} Q_{t}\left(Q_{B}^{\alpha} u \wedge u_{B}^{\alpha}\right) \leq Q_{B}^{\alpha} u \wedge u_{B}^{\alpha} \mathfrak{m}$-a.e. for all $t>0$, and we have $Q_{B}^{\alpha} u \geq u$ q.e. on $B$, which implies that $Q_{B}^{\alpha} u \geq u_{B}^{\alpha} \mathfrak{m}$-a.e., as mentioned above; consequently, $Q_{B}^{\alpha} u=u_{B}^{\alpha} \mathfrak{m}$-a.e. The proof of (7.7) is easy, and the proof of $(7.6)$ is similar to the proof for $[8,(4.3 .3)]$, so we omit it.

Next, we only assume that $u \in \mathcal{F} \cap \mathscr{B}(E)$. Fix $\alpha>0$. For $n \in \mathbb{N}$ and $g_{n}:=n\left(I-n S_{n+\alpha}\right) u \in L^{2}(E ; \mathfrak{m}) \cap \mathscr{B}(E)$, we have $u_{n}:=S_{\alpha} g_{n}=n S_{n+\alpha} u(\in$ $\mathcal{F}) \rightarrow u$ as $n \rightarrow \infty$ in $\mathcal{Q}_{\alpha}$, and equivalently in $\mathcal{Q}_{\beta}$ for $\beta>0$, hence in $\mathcal{E}_{1}$. By taking a subsequence, we have the convergence $u_{n} \rightarrow u$ as $n \rightarrow \infty$ q.e., so $Q_{B}^{\alpha} u_{n} \rightarrow Q_{B}^{\alpha} u$ as $n \rightarrow \infty$ q.e. Putting $u_{n, k}:=u_{n}-u_{k}$ and $g_{n, k}:=g_{n}-g_{k}$, we then have that

$$
\begin{aligned}
\mathcal{Q}_{\alpha}\left(Q_{B}^{\alpha} u_{n, k}, Q_{B}^{\alpha} u_{n, k}\right) & =\mathcal{Q}_{\alpha}\left(S_{\alpha} g_{n, k}-S_{\alpha}^{D} g_{n, k}, S_{\alpha} g_{n, k}-S_{\alpha}^{D} g_{n, k}\right) \\
& =\left(g_{n, k}, S_{\alpha} g_{n, k}-S_{\alpha}^{D} g_{n, k}\right)_{\mathfrak{m}} \\
& \leq\left(g_{n, k}, S_{\alpha} g_{n, k}\right)_{\mathfrak{m}}=\mathcal{Q}_{\alpha}\left(u_{n, k}, u_{n, k}\right) \rightarrow 0
\end{aligned}
$$


as $n, k \rightarrow \infty$. Here $S_{\alpha}^{D} f(x):=\mathbf{E}_{x}\left[\int_{0}^{\tau_{D}} e^{-\alpha s} Z_{s} f\left(X_{s}\right) \mathrm{d} s\right]$. Therefore, we obtain $Q_{B}^{\alpha} u \in \mathcal{F}$ and $\mathcal{Q}_{\alpha}\left(Q_{B}^{\alpha} u, v\right)=0$ for $v \in \mathcal{F}_{D}$ for each $\alpha>0$. The $\mathcal{E}$-quasicontinuity of $Q_{B}^{\alpha} u$ follows from that $e^{-\alpha t} Q_{t} Q_{B}^{\alpha} u \rightarrow Q_{B}^{\alpha} u$ as $t \rightarrow 0$ q.e. and $\left\{e^{-\alpha t} Q_{t} Q_{B}^{\alpha} u\right\}$ is $\mathcal{E}_{1}$-bounded.

We now prove the latter assertion. Suppose first that $u \in \mathcal{F}$. We then have that $\mathcal{Q}\left(Q_{B}^{\alpha} u, Q_{B}^{\alpha} u\right) \leq \mathcal{Q}_{\alpha}\left(Q_{B}^{\alpha} u, Q_{B}^{\alpha} u\right) \leq C \mathcal{Q}_{\alpha}(u, u)$, where $C$ is a constant independent of $\alpha \in] 0,1]$. From this, we have $\lim _{\alpha \rightarrow 0}\left\|\alpha Q_{B}^{\alpha} u\right\|_{2}=0$ and

$$
\begin{aligned}
c^{-1} \mathcal{E}\left(u-\mathcal{Q}_{B}^{\alpha} u, u-\mathcal{Q}_{B}^{\alpha} u\right) & \leq \mathcal{Q}\left(u-\mathcal{Q}_{B}^{\alpha} u, u-\mathcal{Q}_{B}^{\alpha} u\right) \\
& \leq \mathcal{Q}_{\alpha}\left(u-\mathcal{Q}_{B}^{\alpha} u, u-\mathcal{Q}_{B}^{\alpha} u\right) \leq 2 C \mathcal{Q}_{\alpha}(u, u)
\end{aligned}
$$

which implies that $u-Q_{B} u \in\left(\mathcal{F}_{D}\right)_{e}$ and $\mathcal{Q}\left(Q_{B} u, v\right)=0$ for $v \in \mathcal{F}_{D}$ by use of the Banach-Saks theorem with $\lim _{\alpha \rightarrow 0}\left(\alpha Q_{B}^{\alpha} u, v\right)_{\mathfrak{m}}=0$. The proof of the assertion for $u \in \mathcal{F}_{e}$ is similar to [8, proofs of Lemma 4.3 .2 and Theorem 4.3.2], so we omit it.

TheOREM 7.4. Suppose that $u \in \mathcal{F}_{e}$ is $\mathcal{Q}$-harmonic on $D$ in the sense that $\mathcal{Q}(u, v)=0$ for any $v \in \mathcal{F}_{D}$. Then we have

$$
u(x)=\mathbf{E}_{x}\left[Z_{\tau_{D}} u\left(X_{\tau_{D}}\right)\right] \quad \text { q.e. } x \in D .
$$

Proof. We easily see that $\mathcal{Q}\left(u-Q_{B} u, v\right)=0$ for any $v \in\left(\mathcal{F}_{D}\right)_{e}$. Since $u-Q_{B} u \in\left(\mathcal{F}_{D}\right)_{e}$, we have $\mathcal{E}\left(u-Q_{B} u, u-Q_{B} u\right)=0$; hence, $u=Q_{B} u$ q.e. on $D$ under (7.5).

Corollary 7.3. Suppose that $(\mathcal{E}, \mathcal{F})$ is a local Dirichlet form on $L^{2}(E ; \mathfrak{m})$ and that $u \in \mathcal{F}_{\text {loc }}$ is $\mathcal{Q}$-harmonic on $D$ in the sense that $\mathcal{Q}(u, v)=0$ for $v \in \mathcal{F}_{D} \cap C_{0}(D)$. Then we have (7.8).

Proof. Let $G$ be an open set such that $\bar{D} \subset G \subset \bar{G} \subset E$. We can take $u_{G} \in \mathcal{F}$ with $u=u_{G}$ m-a.e. on $G$. Then $\mathcal{Q}(u, v)=\mathcal{Q}\left(u_{G}, v\right)=0$ for $v \in \mathcal{F}_{D} \cap$ $C_{0}(D)$ implies that $u_{G}(x)=\mathbf{E}_{x}\left[Z_{\tau_{D}} u_{G}\left(X_{\tau_{D}}\right)\right]$ q.e. $x \in D$. Since $\mathbf{P}_{x}\left(X_{\sigma_{D^{c}}} \notin\right.$ $\left.\partial D, \sigma_{D^{c}}<\infty\right)=0$ q.e. $x \in D$, we obtain the assertion.

Corollary 7.4. Suppose that $(\mathcal{E}, \mathcal{F})$ is a local Dirichlet form on $L^{2}(E$; $\mathfrak{m})$. Then $\mathbf{E}_{x}\left[Z_{\tau_{D}}: \tau_{D}<\zeta\right]<\infty$ for q.e. $x \in D$.

Proof. Let $G$ be an open set such that $\bar{D} \subset G \subset \bar{G} \subset E$. Taking $e_{G} \in$ $\mathcal{F}$ with $e_{G}=1$ q.e. on $G$, we have $\mathbf{E}_{x}\left[Z_{\tau_{D}}: \tau_{D}<\zeta\right]=Q_{B} e_{G}(x)<\infty$ q.e. $x \in D$. 
REMARK 7.2. In [7], the Dirichlet boundary value problem for an elliptic operator on Euclidean space associated with a quadratic form obtained from lower-order perturbations is solved for bounded Lipschitz domains. Our condition (7.5), which can be regarded as conditions for coefficients of the elliptic operator in the framework of [7], is different from what is treated in $[7]$.

EXAMPLE 7.1. Let $\mathbf{X}$ be the $d$-dimensional Brownian motion, and let $\left(\mathcal{E}, H^{1}\left(\mathbb{R}^{d}\right)\right)$ be the associated Dirichlet form on $L^{2}\left(\mathbb{R}^{d}\right)$. Here $H^{1}\left(\mathbb{R}^{d}\right)$ is the 1-order Sobolev space, and $\mathcal{E}(f, g):=(1 / 2) \mathbf{D}(f, g)=(1 / 2) \int_{\mathbb{R}^{d}} \nabla f(x)$. $\nabla g(x) \mathrm{d} x$ for $f, g \in H^{1}\left(\mathbb{R}^{d}\right)$. Assume that $d \geq 3$, and fix a bounded domain $D$. Let $b, \widehat{b}: \mathbb{R}^{d} \rightarrow \mathbb{R}^{d}$ be measurable vector fields, and let $c: \mathbb{R}^{d} \rightarrow \mathbb{R}$ be a measurable function such that $|b|^{2},|\widehat{b}|^{2},|c| \in L^{p}\left(\mathbb{R}^{d}\right) \cap L^{1}\left(\mathbb{R}^{d}\right)$ for $p>d / 2$. Then $|b|^{2}(x) \mathrm{d} x,|\widehat{b}|^{2}(x) \mathrm{d} x,|c|(x) \mathrm{d} x \in \mathbf{K}(\mathbf{X}) \cap L^{1}\left(\mathbb{R}^{d}\right) \subset \mathbf{K}_{\infty}(\mathbf{X})$, where $\mathbf{K}_{\infty}(\mathbf{X})$ is the family of Green-tight measures of Kato class (see [23]). Since any $\nu \in \mathbf{K}_{\infty}(\mathbf{X})$ is Green-bounded by [2, Proposition 2.2], we have

$$
\sup _{x \in \mathbb{R}^{d}} \mathbf{E}_{x}\left[\int_{0}^{\infty}\left(|b|^{2}+|\widehat{b}|^{2}+|c|\right)\left(X_{s}\right) \mathrm{d} s\right]<\infty .
$$

By scaling if necessary, we can take $b, \widehat{b}, c$ so that

$$
\begin{aligned}
& \sqrt{2\left\|\mathbf{E} \cdot\left[\int_{0}^{\infty}|b|^{2}\left(X_{s}\right) \mathrm{d} s\right]\right\|_{\infty}}+\sqrt{2\left\|\mathbf{E} \cdot\left[\int_{0}^{\infty}|\widehat{b}|^{2}\left(X_{s}\right) \mathrm{d} s\right]\right\|_{\infty}} \\
& +\left\|\mathbf{E} \cdot\left[\int_{0}^{\infty}|c|\left(X_{s}\right) \mathrm{d} s\right]\right\|_{\infty}<1 .
\end{aligned}
$$

Define $M_{t}:=\int_{0}^{t} b\left(X_{s}\right) \mathrm{d} X_{s}, \widehat{M}_{t}:=\int_{0}^{t} \widehat{b}\left(X_{s}\right) \mathrm{d} X_{s}$, and $A_{t}:=\int_{0}^{t} c\left(X_{s}\right) \mathrm{d} s$. Then the Revuz measures associated with $\langle M\rangle,\langle\widehat{M}\rangle$, and $A$ are given by $\mu_{\langle M\rangle}(\mathrm{d} x)=|b|^{2}(x) \mathrm{d} x, \mu_{\langle\widehat{M}\rangle}(\mathrm{d} x)=|\widehat{b}|^{2}(x) \mathrm{d} x$, and $\mu(\mathrm{d} x)=c(x) \mathrm{d} x$. Consider the quadratic from $\left(\mathcal{Q}, H^{1}\left(\mathbb{R}^{d}\right)\right)$ obtained by

$$
\begin{aligned}
\mathcal{Q}(f, g)= & \frac{1}{2} \mathbf{D}(f, g)-\int_{\mathbb{R}^{d}} f(x)\langle\nabla g(x) \cdot \widehat{b}(x)\rangle \mathrm{d} x \\
& -\int_{\mathbb{R}^{d}} g(x)\langle\nabla f(x) \cdot b(x)\rangle \mathrm{d} x-\int_{\mathbb{R}^{d}} f(x) g(x) c(x) \mathrm{d} x
\end{aligned}
$$

for $f, g \in H^{1}\left(\mathbb{R}^{d}\right)$. We obtain that, if $u \in H_{\text {loc }}^{1}\left(\mathbb{R}^{d}\right)$ satisfies $\mathcal{Q}(u, v)=0$ for any $v \in C_{0}^{\infty}(D)$, then $u(x)=\mathbf{E}_{x}\left[Z_{\tau_{D}} u\left(X_{\tau_{D}}\right)\right]$ q.e. $x \in D$ by Corollary 7.3. 
Here $Z_{t}\left(t \in\left[0, \infty[)\right.\right.$ is defined by $Z_{t}:=\exp \left(A_{t}+M_{t}-\frac{1}{2}\langle M\rangle_{t}\right) \exp \left(\widehat{M}_{t} \circ r_{t}-\right.$ $\left.\frac{1}{2}\langle\widehat{M}\rangle_{t}\right)$

Acknowledgments. Kuwae's work was partially supported by Grant-inAid for Scientific Research (B) 22340036 from the Japan Society for the Promotion of Science.

\section{REFERENCES}

[1] C. Berg and G. Forst, Potential Theory on Locally Compact Abelian Groups, Ergeb. Math. Grenzgeb. 87, Springer, New York, 1975. MR 0481057.

[2] Z.-Q. Chen, Gaugeability and conditional gaugeability, Trans. Amer. Math. Soc. 354 (2002), no. 11, 4639-4679. MR 1926893. DOI 10.1090/S0002-9947-02-03059-3.

[3] Z.-Q. Chen, P. J. Fitzsimmons, K. Kuwae, and T.-S. Zhang, Perturbation of symmetric Markov processes, Probab. Theory Related Fields 140 (2008), 239-275. MR 2357677. DOI 10.1007/s00440-007-0065-2.

[4] - Stochastic calculus for symmetric Markov processes, Ann. Probab. 36 (2008), 931-970; Correction, Ann. Probab. 40 (2012), 1375-1376. MR 2408579; MR 2962095. DOI 10.1214/07-AOP347.

[5] - On general perturbation of symmetric Markov processes, J. Math. Pures Appl. (9) 92 (2009), 363-374. MR 2569183. DOI 10.1016/j.matpur.2009.05.012.

[6] Z. Q. Chen, Z. M. Ma, and M. Röckner, Quasi-homeomorphisms of Dirichlet forms, Nagoya Math. J. 136 (1994), 1-15. MR 1309378.

[7] Z.-Q. Chen and T. Zhang, Time-reversal and elliptic boundary value problems, Ann. Probab. 37 (2009), 1008-1043. MR 2537548. DOI 10.1214/08-AOP427.

[8] M. Fukushima, Y. Ōshima, and M. Takeda, Dirichlet Forms and Symmetric Markov Processes, de Gruyter Stud. Math. 19, de Gruyter, Berlin, 2011. MR 1303354. DOI 10.1515/9783110889741.

[9] S. W. He, J. G. Wang, and J. A. Yan, Semimartingale Theory and Stochastic Calculus, Science Press, Beijing, 1992. MR 1219534.

[10] J. Jacod and A. N. Shiryaev, Limit Theorems for Stochastic Processes, Grundlehren Math. Wiss. 288, Springer, Berlin, 1987. MR 0959133. DOI 10.1007/978-3-662-02514-7.

[11] K. Kuwae, Functional calculus for Dirichlet forms, Osaka J. Math. 35 (1998), 683715. MR 1648400.

[12] — Maximum principles for subharmonic functions via local semiDirichlet forms, Canad. J. Math. 60 (2008), 822-874. MR 2432825. DOI 10.4153/CJM-2008-036-8.

[13] - Stochastic calculus over symmetric Markov processes without time reversal, Ann. Probab. 38 (2010), 1532-1569. MR 2663636. DOI 10.1214/09-AOP516.

[14] - Errata to Stochastic calculus over symmetric Markov processes without time reversal, Ann. Probab. 40 (2012), 2705-2706. MR 2663636. DOI 10.1214/09-AOP516.

[15] T. J. Lyons and T. S. Zhang, Decomposition of Dirichlet processes and its application, Ann. Probab. 22 (1994), 494-524. MR 1258888. 
[16] T. J. Lyons and W. A. Zheng, "A crossing estimate for the canonical process on a Dirichlet space and a tightness result" in Colloque Paul Lévy sur les Processus Stochastiques (Palaiseau, 1987), Astérisque 157-158, Soc. Math. France, Paris, 1988, 249-271. MR 0976222.

[17] Z. M. Ma and M. Röckner, Introduction to the Theory of (Nonsymmetric) Dirichlet Forms, Springer, Berlin, 1992. MR 1214375. DOI 10.1007/978-3-642-77739-4.

[18] S. Nakao, Stochastic calculus for continuous additive functionals of zero energy, Z. Wahrsch. Verw. Gebiete 68 (1985), 557-578. MR 0772199. DOI 10.1007/BF00535345.

[19] K. Sato, Lévy Processes and Infinitely Divisible Distributions, Cambridge Stud. Adv. Math. 68, Cambridge University Press, Cambridge, 1999. MR 1739520.

[20] M. Sharpe, General Theory of Markov Processes, Pure Appl. Math. 133, Academic Press, Boston, 1988. MR 0958914.

[21] M. Takeda, "On exit times of symmetric Lévy processes from connected open sets" in Probability Theory and Mathematical Statistics (Tokyo, 1995), World Scientific, River Edge, NJ, 1996, 478-484. MR 1467965.

[22] J. B. Walsh, Markov processes and their functionals in duality, Z. Wahrsch. Verw. Gebiete 24 (1972), 229-246. MR 0329056.

[23] Z. Zhao, Subcriticality and gaugeability of the Schrödinger operator, Trans. Amer. Math. Soc. 334, no. 1 (1992), 75-96. MR 1068934. DOI 10.2307/2153973.

Department of Mathematics and Engineering

Graduate School of Science and Technology

Kumamoto University

Kumamoto

860-8555 Japan

kuwae@gpo.kumamoto-u.ac.jp

Current:

Department of Applied Mathematics

Faculty of Science

Fukuoka University

Fukuoka

814-0180 Japan

kuwae@fukuoka-u.ac.jp 